University of Louisville

ThinkIR: The University of Louisville's Institutional Repository

Electronic Theses and Dissertations

$12-2011$

\title{
Health disparities and depression in rural and urban older adults.
}

Jennifer Ann Zimmerman

University of Louisville

Follow this and additional works at: https://ir.library.louisville.edu/etd

\section{Recommended Citation}

Zimmerman, Jennifer Ann, "Health disparities and depression in rural and urban older adults." (2011). Electronic Theses and Dissertations. Paper 1650.

https://doi.org/10.18297/etd/1650

This Doctoral Dissertation is brought to you for free and open access by ThinkIR: The University of Louisville's Institutional Repository. It has been accepted for inclusion in Electronic Theses and Dissertations by an authorized administrator of ThinkIR: The University of Louisville's Institutional Repository. This title appears here courtesy of the author, who has retained all other copyrights. For more information, please contact thinkir@louisville.edu. 


\title{
HEALTH DISPARITIES AND DEPRESSION IN RURAL AND URBAN OLDER ADULTS
}

\author{
By \\ Jennifer Ann Zimmerman \\ B.A. Marist College, 2004 \\ M.A. University of Louisville, 2008

\begin{abstract}
A Dissertation
Submitted to the Faculty of the

College of Arts and Sciences of the University of Louisville in Partial Fulfillment of the Requirements

for the Degree of
\end{abstract} \\ Doctor of Philosophy \\ Department of Psychological and Brain Sciences \\ University of Louisville \\ Louisville, KY
}

December 2011 



\title{
HEALTH DISPARITIES AND DEPRESSION IN RURAL AND URBAN OLDER
} ADULTS

\author{
By \\ Jennifer Ann Zimmerman \\ B.A., Marist College, 2004 \\ M.A., University of Louisville, 2008
}

A Dissertation Approved on

July 27, 2010

By the following Dissertation Committee:

Dr. Benjamin Mast

Dissertation Director 


\section{DEDICATION}

This dissertation is dedicated to:

My brother, Ryan Matthew Zimmerman

My great grandmother, Victoria Jean Corcoran

$\&$

One of my greatest educational supporters, Douglas C. Birdsall 


\section{ACKNOWLEDGMENTS}

I would first and foremost like to thank my mentor, Dr. Benjamin Mast, for all of his support, guidance, and encouragement throughout my graduate student career. I do not take for granted how lucky I am to have such a wonderful mentor and role model. I would also like to thank the many other clinical supervisors and faculty members who have supported and challenged me over the past four years, and who provided me with opportunities to learn and grow. Many thanks to my dissertation committee members for your willingness to support me on this project. I would like to thank my lab mates; I could not have asked for better friends. I would like to thank Todd, for moving with me to Louisville so I could follow my dream and for supporting that dream for the past four years. Finally, I would like to thank my family for their ability to provide so much support and encouragement even across the distance. 


\title{
ABSTRACT \\ HEALTH DISPARITIES AND DEPRESSION IN RURAL AND URBAN OLDER ADULTS
}

\author{
Jennifer Ann Zimmerman
}

July 27,2010

Depression is one of the most prevalent psychiatric conditions experienced by older adults and represents a major public health concern. Rural/urban residence may affect the prevalence of depression as rural older adults differ from their urban counterparts in many respects. One important difference found in the literature is that rural OAs are often faced with more health disparities (HDs) compared to their urban counterparts. The current study investigated the association between HDs and depression in a sample of rural and urban OAs, and examined whether HDs contribute to our understanding of how rurality impacts the prevalence and severity of depression. It was hypothesized that rural OAs would report a greater severity of depression compared to urban OAs, and that a greater severity of HD in rural areas would account for this difference. This study utilized a baseline sample of 3,996 older adults from the Duke Established of Populations for Epidemiologic Studies of the Elderly (EPESE): Piedmont Health Survey of the Elderly. Participants were stratified into separate groups based on level of rurality as defined by the Economic Research Service's Rural-Urban Continuum Codes. The main, overarching hypothesis was not 
supported by the current study. Level of rurality, in and of itself, was not significantly associated with depression severity or rates of prevalent or incident depression, and other than healthcare utilization, the HD variables investigated (overall medical burden, cumulative vascular risk, and negative health behaviors) did not differ by level of rurality. Although rural/urban status did not directly affect HDs and depression, followup analyses indicated this was likely due to the heterogeneity of individuals within rural and urban counties. This finding indicates that the inclusion of individual characteristics may contribute to the understanding of differences in depression between rural and urban OAs. The relationship between level of rurality and depression is more complex than a simple rural/urban distinction and other variables (e.g. ethnicity, income, and place of usual outpatient healthcare services) need to be investigated in conjunction with place of residence to understand how HDs and depression differ across rural and urban groups. 
TABLE OF CONTENTS

\section{PAGE}

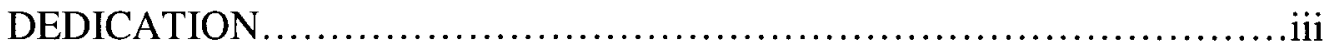

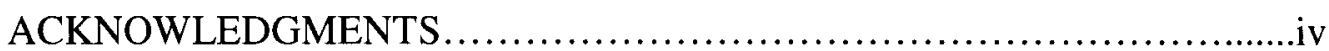

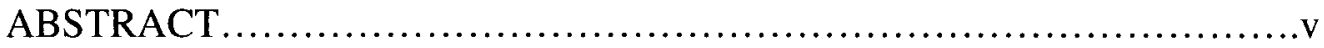

LIST OF TABLES ........................................................

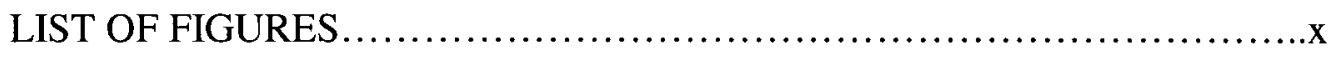

LIST OF ACRONYMS and COUNTY CODE DESCRIPTIONS.....................xi

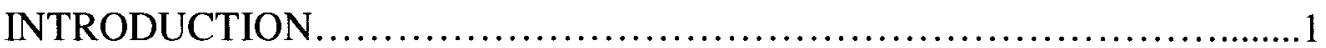

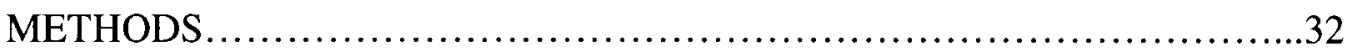

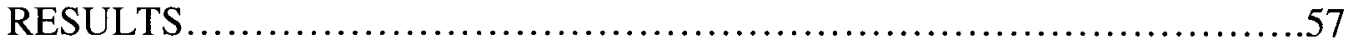

DISCUSSION .................................................................. 75

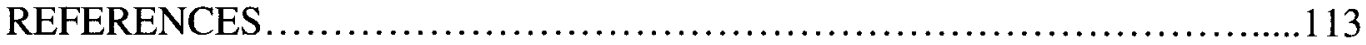

APPENDICES ............................................................

CURRICULUM VITAE................................................ 170 


\section{LIST OF TABLES}

TABLE

PAGE

1. Description of health disparities investigated.................................137

2. Rural/urban continuum codes by Duke EPESE county $\ldots \ldots \ldots \ldots \ldots \ldots \ldots \ldots \ldots 138$

3. Description of the ERS Rural/Urban Continuum Codes..........................139

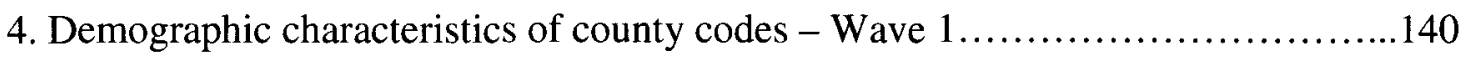

5. Association between study demographic variables and depression (mean CES-D score)

6. Percent of participants by level of HDs and Mean (SD) of HD by total sample

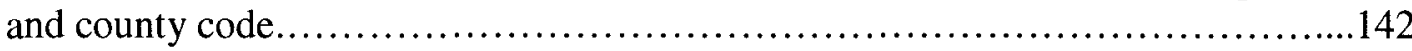

7. Follow-up post hoc analyses: Healthcare utilization and county code...............143

8. Place of usual healthcare by county code: private practice, public clinic, and nowhere.

9. Differences between participants who use private practice offices vs. public clinics

10. Satisfaction with healthcare services by county code.............................146

11. Differences on HCU between county codes 2 and 9 Blacks and Non-Hispanic Whites.

12. Percentage of Blacks and Non-Hispanic Whites by place of usual outpatient healthcare

13. Percent of Black and Non-Hispanic White residents with prevalent depression (CES-D > 9) by County Code.

14. Perceived social support, overall medical burden, county code, and depression.

15. Perceived social support, healthcare utilization, county code, and 
depression.

16. Percentage of Blacks and Non-Hispanic Whites reporting low perceived social support (score $=2$ or 3 ) by county code.

17. Physical disability (PD), overall medical burden, county code, and depression

18. Physical disability (PD), healthcare utilization, county code, and depression. 154

19. Differences in self-efficacy and negative attitudes towards doctors by

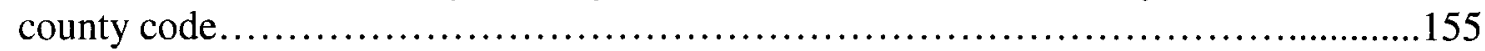

20. Self efficacy and overall medical burden.........................................156

21. Rates of incident depression (CES-D $\geq 9$ ) at follow-up by county code 157 


\section{LIST OF FIGURES}

FIGURE

PAGE

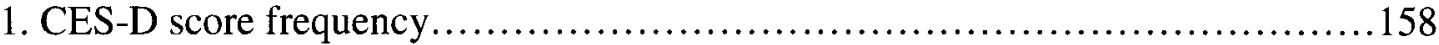

2. Mean depression score for levels of overall medical burden by county code.......159

3. Mean depression score for levels of HCU by county code........................160

4. Mean depression score for levels of NHBs by county code.......................161

5. Mean depression score for levels of CVR by level of rurality....................162

6. Percent of Blacks and Non-Hispanic Whites with prevalent depression

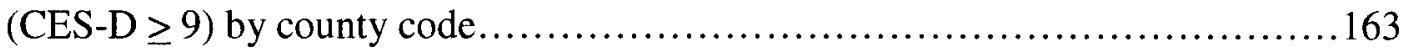




\section{LIST OF ACRONYMS and COUNTY CODE DESCRIPTIONS}

\section{ACRONYMS}

OAs - Older Adults

HDs - Health Disparities

HCU - Healthcare Utilization

NHBs - Negative Health Behaviors

CVR - Cumulative Vascular Risk

VRF - Vascular Risk Factor

NAs - Negative Attitudes towards Doctors

COUNTY CODES (in order from most urban to most rural)

Code 2: The most urban county code in the current study (large county in a metropolitan area)

Code 6: Small urban county (suburban) that is adjacent to a large, metropolitan county

Code 7: Small urban county (suburban) that is not adjacent to a metropolitan county

Code 8 : Rural county that is adjacent to a metropolitan area

Code 9: The most rural county code, both in the ERS code continuum and in the current study; smallest population size and not adjacent to a metropolitan county

a. Code 8 is used in Wave 2 data analyses only. 


\section{INTRODUCTION}

Older adults (OAs) age 65 and older represent approximately $12 \%$ of the United States population (Shear, Ginsberg, Roose, Lenze, Alexopoulos, \& Hollander, 2005). The population of OAs is growing at a rapid rate, and by the year $2030,20 \%$ of the U.S. population will be at least 65 years old (Moore, Moir, \& Patrick, 2004). Depression is one of the most prevalent psychiatric conditions experienced by OAs (Blazer, 2003), and late life depression (LLD) is a major public health concern (Lebowitz, Pearson, Schneider, et al., 1997). Depression significantly reduces the quality of life of OAs and leads to a number of negative outcomes, such as a decrease in well-being, increased mortality, increased functional impairment, and increased service utilization (Blazer, 2003). Studies have reported prevalence rates of LLD ranging from $8-16 \%$ in community-dwelling samples in the U.S. (Blazer \& George, 1987 Lawhorne, 2005; Lyness, King, Cox, Yoediono, \& Caine, 1999; Mojtaba \& Olfson, 2004).

The U.S. population of OAs is heterogeneous with regard to socioeconomic status (SES), ethnicity, and place of residence. Research has shown that such heterogeneity can greatly affect the prevalence of depression in OA samples and can lead to difficulty detecting, diagnosing, preventing, and treating depression (Kales \& Valenstien, 2002). Given the diversity within the U.S. OA population, in order to better understand the prevalence of depression and the factors that influence LLD, investigators have argued for research in more specific OA populations (Beekman, Copeland, \& Prince, 1999). By understanding the differences in 
factors associated with depression in different OA populations (e.g. community vs. assisted living vs. nursing homes), researchers and public policy makers can begin to investigate and create more tailored and effective prevention and treatment initiatives.

Rural OAs represent one unique sub-population of U.S. elders. Currently, $87 \%$ of all US territory is considered rural and $21 \%$ of the U.S. population lives in rural areas (Cromartie \& Bucholtz, 2008; Klugman, 2008). According to the CDC Rural and Urban 2001 Health Chartboook, the age structure of the population tends to get older as rurality increases, a trend which is present in all U.S. geographical regions (Ebernhardt, Ingram, Makuc, Pamuk, Fried, Harper, Schoenbom, \& Xia, 2001). As of 2004, approximately 7.5 million U.S. elders age 65 and older lived in a nonmetropolitan area (Rural Population and Migration, 2007).

Although rural elders represent a unique and growing population, there appears to be an urban bias in gerontological research, with few studies focusing specifically on rural OAs (Coward \& Lee, 1984; O’Hara, Kohout, \& Wallace, 1985; Rokke \& Klenow, 1998). Further, research on the mental health needs of rural OAs is very scarce with little research investigating the variables associated with depression in this population (Rokke \& Klenow, 1998). In addition to the lack of research, the current literature on depression in rural residents reveals conflicting results and limitations that threaten the generalizability and validity of the research findings (St John, Blandford, \& Strain, 2002).

The current literature lacks studies investigating variables associated with rural/urban residence that are related to differences in depression rates and severity. One rural/urban difference that may be particularly important in depression research is differences in health disparities (HDs). Compared to urban residents, those living in rural 
areas may experience a more HDs, such as less access to care, higher prevalence of chronic medical conditions, and negative health risk behaviors (e.g. smoking). Given a greater prevalence of HDs in rural areas, it is possible that such differences are associated with an increased prevalence and risk for depression in rural compared to urban OAs. Investigating differences in the HDs present in rural versus urban locations and their relationship to depression prevalence is particularly important given the high prevalence of HDs facing rural residents (Ebernhardt \& Pamuk, 2004). This study explored the association between HDs and depression in rural and urban OAs, and examined whether HDs contribute to our understanding of how rurality impacts depression severity and prevalence of depression.

\section{Depression in Rural Adults}

A thorough review of literature examining depression in rural residents revealed little has been investigated in this area. Twenty-four studies were found investigating depression in U.S. rural adults. These 24 studies can be broken down into four types; 1) 9 studies investigating depression in rural adults $15+$ or older; 2) 5 studies investigating depression in rural OAs 59+ or older; 3) 8 studies comparing depression in rural versus urban adults age 18+ or older; and 4) 2 studies comparing depression in rural and urban OAs, $55+$ or older.

Based on the data in the above studies, it is apparent that prevalence rates of depression found in rural OA samples are highly variable. Prevalence rates found in studies using a self-report measure assessing depressive symptomotology in rural OAs ranged from 5.5\% to 28\% (Buys, Roberto, Miller, Blieszner, 2008; Murrell, Himmelfarb, \& Wright, 1983; O’Hara, Kohout, \& Wallace, 1985; Rokke \& Klenow, 1998; Schulman, 
Gairola, Kuder, \& McCulloch, 2002; Swenson, Baxter, Shetterly, et al., 2000; Wallace \& O'Hara, 1992). The one study that used diagnostic criteria for Major Depression in a rural OA sample found the prevalence rate of Major Depression to be 1.2\% (O'Hara, Kohout, \& Wallace, 1985). The remaining studies investigating prevalence rates of rural individuals age 15 or older found that $0.6 \%$ to $49.1 \%$ reported significant depressive symptomatology (Carruth \& Logan, 2002; Comstock \& Helsing, 1976; Hauenstein \& Boyd, 1994; Hauenstein, \& Peddada, 2007; Lane, Shellenberger, Gresen, \& Moore, 2000; McCrone, Cotton, Jones, et al., 2007; Muntaner \& Barnett, 2000). Prevalence rates of Major Depression assessed via structured diagnostic interviews in rural adults (age 15+) ranged from $1.1 \%$ to $39 \%$ (Blazer, George, Landerman, et al., 1985; Crowell, George, Blazer, \& Landerman, 1986; Probst, Laditka, Charity, et al., 2006; Schwab, Warheit, \& Holzer, 1974; Sears, Danda, Evans, 1999; Simmons \& Havens, 2007). Given the results presented above, it is surprising that many articles state the prevalence rates of depression are lower in rural compared to urban samples (Rokke \& Klenow, 1998). An analysis of the results from the aforementioned 24 studies reveals no consensus as to whether prevalence rates of depression are greater in rural versus urban settings, which is likely the result of different measurement techniques utilized and differences in the definition of rural, which will be explained in more detail below.

To date, only 7 of the 24 studies identified specifically investigate depression in rural OAs, which represents a significant gap in the literature. While stressors associated with rural life may affect all rural residents regardless of age, they may not affect all rural individuals equally. Experiences and conditions associated with aging may predispose OAs to certain risk factors for depression, such as increased health problems, increased 
disability, limited mobility, fewer supports, etc. (Harman, Edlund, Fortney, \& Kallas, 2005; Lawhorne, 2005). These factors, combined with the greater rates of HDs found in rural areas, may lead to greater rates of depression in rural versus urban OAs, making rural OAs an important population to study in depression research. Therefore, studies investigating the factors associated with depression, specifically in rural OAs, are necessary.

Variables found to be significantly associated with depression in at least one of the seven rural OA studies identified include: lower income, less education, living alone, having been widowed, divorced, or separated, greater alcohol use, poorer cognitive status, poorer perceived physical health, a greater number of chronic medical conditions, dissatisfaction with social support, smaller household size, less frequent group participation, taking depression medication, and being female (Buys, Roberto, Miller, Blieszner, 2008; O’Hara, Kohout, \& Wallace, 1985; Rokke \& Klenow, 1998; Swenson, Baxter, Shetterly, et al., 2000; Wallace \& O’Hara, 1992).

Focusing on prevalence rates alone, however, or looking at simple regression or correlational analyses solely in a rural sample does not provide the information necessary to investigate differences in the factors associated with rural versus urban depression risk. Rural/urban comparative studies are needed to investigate differences in depression between these populations, which would provide information that could be used to develop tailored prevention and intervention programs for OAs living in certain geographical areas (Scheidt, 1984). To date, variables associated with place of residence that relate to depression have been highly understudied. Only one study (Probst et al., 2006) has compared independent variables associated with depression between rural and 
urban populations, making it difficult to identify depression risk factors in rural versus urban communities. Probst and colleagues (2006) found that rural compared to urban residents were more likely to be married, and on average, were more likely to be unemployed, have lower incomes, and have attained lower educational achievement. Rural residents were also more likely to report poorer health, worsening health over the past year, and a greater prevalence of the health conditions assessed (obesity, diabetes, hypertension, and asthma) compared to their urban counterparts. No study to date has compared factors associated with depression between rural and urban older adults.

In addition to a lack of research, there is a great deal of inconsistency in the available literature on depression in U.S. rural residents. One example is the wide range of reported prevalence rates of depression in rural residents previously outlined. The main reason for the inconsistency in depression rates is the lack of standardization across such studies (Scheidt, 1984). The 24 studies reviewed here utilized differing samples and sampling strategies, were conducted in various settings, and used different methods to assess depression (Scheidt, 1984; St. John, Blandford, \& Strain, 2006). One of the most significant limitations in the extant literature on rural depression is that currently, there is no standard definition of rural.

\section{Definition of "Rural" and "Urban"}

The definition of rural varies significantly in the current literature, and many studies fail to provide an operational definition for their rural and/or urban samples. Fewer than 10 of the 24 studies reviewed here defined rural based on a formal classification system. These include the Office of Management and Budget's (OMB) standard metropolitan statistical area (SMSA) classification guidelines (Blazer, et al., 
1985; Blazer, Kessler, McGonagle, Swarts, 1994; Crowell, et al., 1986; Murrell, Himmelfarb, \& Wright, 1983; Probst, et al., 2006), the U.S. Census Bureau guidelines (Schulman, et al, 2002), and the U.S. Department of Agriculture - Economic Research Service guidelines (Kessler, Berglund, Demler, et al., 2003; Simmons \& Havens, 2007). Further, the compositions of the study samples are highly variable and many "rural" samples actually include participants from non-rural locations. For example, $44 \%$ of Rokke \& Klenow's (1998) sample consists of participants living in a city with a population between 10,000 and 50,000; and Neff's (1983) rural sample is made up of 4 counties considered $59 \%$ urban.

The most commonly used formal definition of rural in research studies to date is the OMB's definition, which is based on their classification of Metropolitan Statistical Areas (MSAs). An MSA is defined as "an area containing a recognized population nucleus and adjacent communities that have a high degree of integration with that nucleus (Spotila, 2000, p. 82228)." Based on this classification system, the OMB defines urban areas as locations within a MSA, and rural areas as anything outside a MSA (i.e. metropolitan vs. non-metropolitan; Stamm, Lambert, Piland, \& Speck, 2007). Unfortunately, this dichotomous, county-based system, often misclassifies areas. Fourteen percent of residents the OMB classifies as metropolitan are classified as rural by the U.S. Bureau of the Census (Washington State Department of Health, 2009). Further, this definition equates to a dichotomous variable, which is not very informative.

"The rurality of a place depends on several factors, including population density or sparseness, measure of settlement size, remoteness from urban areas, access to services, land use, main employment groups, the population's economic activity, and 
socioeconomic characteristics (Stamm, Lambert, Piland, \& Speck, 2007, p. 299)," suggesting a dichotomous measure of rurality, based on population density alone, is not accurate or informative. A more standardized definition of rural with greater specificity is clearly needed. This is particularly true when investigating healthcare factors, as rural counties within MSAs may be very different from rural counties outside of MSAs in terms of access to healthcare resources, individual healthcare utilization patterns, cultural beliefs, etc.

None of the aforementioned 24 studies investigating rural depression have sufficiently differentiated groups within the rural/urban continuum. Utilizing a more detailed coding system would allow investigators to compare small, isolated, rural counties, to larger rural counties close to an urban center. This would provide more useful information when studying $\mathrm{HD}$ variables because difference in proximity to urban centers is particularly relevant when studying the effects of rural HDs, such as contact with healthcare providers and rural culture, on depression. It is possible there would be significant differences in the present HD factors, and those associated with depression in rural communities that are 15-30 minutes from an urban center with multiple healthcare facilities compared to rural communities 4+ hours from any healthcare specialist (Washington State Department of Health, 2009).

The current study is the first study to utilize a more specific and detailed rural/urban classification system to investigate differences in depression based on level of rurality. This study utilized the U.S. Department of Agriculture's, Economic Research Service (ERS) Rural/Urban Continuum Codes system, which is particularly useful for investigating the association between HDs and depression based on geographic region. 
This system is a 9-tiered classification system based on U.S. census tract geography (See Table 3). Codes 1-3 represent large metropolitan, urban core areas with populations ranging from 250,000 or greater. Codes 4-7 represent urban populations based on size and proximity to a large metropolitan area (and are comparable to "suburban" areas), and codes 8-9 represent small, rural populations based on proximity to a metropolitan center. These codes provide increased flexibility for researchers, as they can be combined and collapsed for statistical analyses (Washington State Department of Health, 2009). The ERS's rural/urban continuum codes allow for examination of the association between HDs and prevalence of depression in rural and urban OAs using a more accurate and variable measure of rurality. Utilizing these rural/urban continuum codes, which take distance from a metropolitan area into account, is a significant strength of the current study.

\section{Health Disparities and Depression in Rural OAs}

According to Scheidt (1984), research would benefit from a search for explanatory factors responsible for depression in rural versus urban populations. Crowell,

George, Blazer, \& Landerman, (1986) suggest:

"Efforts should be devoted to specifying what it is about rural and urban environments that affect the risk of psychiatric disorder. As operationally defined, the distinction between urban and rural residence is based simply upon population density; although it is possible that the number of people per square mile has a direct effect on mental health, it is more likely that place of residence now serves as a proxy for one or more important social processes that affect the risk of psychiatric disorder (p. 313)."

Identification of these processes would have important implications for understanding the factors that influence differences in prevalence rates of depression in rural versus urban OAs and therefore, for its prevention and treatment. 
To date, the association of factors based on geographical location (rurality/urbanicity) and depression in OAs has been inadequately studied. If differences exist in the frequency and severity of a particular risk factor in rural versus urban areas, an understanding of such differences would allow for mental health service planners to develop more appropriate and effective prevention and treatment interventions. If differences exist in depression rates and severity by rural/urban status, understanding the factors that account for such differences are needed. Rural residence may affect the prevalence of depression as rural individuals differ from their urban counterparts in many respects, particularly in regards to the presence of a number of HD variables. Past research has found that rural OAs are faced with a greater prevalence of HDs compared to their urban counterparts. Given the significant link between physical health and depression, if greater severity and prevalence rates of depression are found in rural versus urban OAs, the more severe HD variables present in rural locations may account for this difference.

Before investigating HDs in more detail, it is important to first define health disparity as it is viewed in the current study and describe the HDs that will be examined. The Centers for Disease Control defines a health disparity population "as a population where there is a significant disparity in the overall rate of disease incidence, prevalence, morbidity, mortality, or survival rates in the population as compared to the health status of the general population (Eberhardt, et al., 2001, p. 1676)." The National Institute of Health defines HDs as "the differences in the incidence, prevalence, mortality, and burden of diseases and other adverse health conditions that exist among specific groups in the United States (as sited in Pamies and Nsiah-Kumi, 2009, p. 2)." The Health 
Resources and Services Administration defines HDs as "population-specific differences in the presence of disease, health outcomes, or access to health care' (as sited in Understanding Health Disparities, p. 3)." Finally, according to Pamies and Nsiah-Kumi (2009), differences between population groups in terms of lifestyle choices and health behaviors is a significant contributor to overall disparities in health outcomes. The current study defines HDs as differences between populations in the prevalence of disease conditions, access to care determined by individual utilization of healthcare resources, and in the prevalence of negative health risk behaviors that contribute to chronic disease (e.g. smoking and physical inactivity).

Research specifically investigating HDs in rural adults and OAs has increased over the last decade and has found that rural compared to urban residents are faced with more HDs, and "fare worse on many dimensions of health compared with populations at other levels of urbanization (Ebernhardt \& Pamuk, 2004, p. 1682)." Current research on rural HDs focuses on four factors: 1) access to care and contact with healthcare providers, 2) disease prevalence, 3) negative health risk behaviors (e.g. level of physical activity/exercise), and 4) culture and health beliefs that may effect health status. These four components encompass the framework for investigating HDs in rural and urban OAs in the current study. The current study is noteworthy in that it investigated all four components of HDs found in the literature. A description of these four HD variables can be found in Table 1.

Access to healthcare is the most frequently studied HD in rural communities (Blazer, Landerman, Fillenbaum, Homer, 1995; Stamm, Lambert, Piland, \& Speck, 2007). Access to care is commonly defined as the number of health care professionals in 
a given area, distance to a primary care physician, specialist, or hospital, and/or lack of insurance coverage (Stamm, Lambert, Piland, et al., 2007). Operationally defined in this manner, access to care primarily represents an environmental HD resulting from the conditions of the community in which one lives.

In addition to such environmental HDs, there is an argument to be made that individual HD variables, such as the health behaviors one chooses to engage in and chronic disease conditions present, are equally important and may be particularly relevant when studying the association between HDs and risk for depression. It is these individual components of HDs that are the focus of the current research study. The individual-level components of HDs investigated in the current study include: 1) healthcare utilization $(\mathrm{HCU})$, measured as the total number of outpatient contacts with healthcare providers in the previous 12 months, 2) overall medical burden (number of chronic medical diseases/conditions), and 3) negative health risk behaviors (NHBs), such as smoking. In addition, cumulative vascular risk (CVR) was investigated as a separate HD variable, as vascular risk factors (VRFs) are investigated as a separate mechanism in the development of depression through brain changes caused by cerebrovascular disease (Alexopoulos, et al., 1997). Finally, differences in cultural attitudes that may affect health behaviors and health outcomes, e.g.. self-efficacy and negative attitudes towards medical doctors (NAs) were investigated. It is possible these culture variables represent one mechanism leading to decreased use of formal preventative and maintenance healthcare services in rural OAs, which could eventually lead to poorer physical health status. It was hypothesized that rural OAs experience a greater prevalence and severity of individual HDs compared 
to urban OAs, and that this differences is related to a greater prevalence and risk for depression in rural versus urban OAs.

This study examined the relationship between HD variables and depression in a sample of rural and urban OAs with the following aims: 1) to investigate the prevalence and severity of HDs in a rural compared to urban OA sample, 2) to investigate the role of HDs on current depression in rural versus urban OAs, 3) to investigate the role of HDs on the risk for developing depression at follow-up in initially depression free rural versus urban OAs, 4) to investigate the effects of potential moderating variables on this relationship, such as perceived social support and physical disability, and 5) to investigate the effects of cultural variables identified with rural residence on healthcare utilization. Social support and physical functioning were chosen as potential moderating variables as these variables have consistently been found to be strong predictors of depression in OAs, and conceptually represent variables that may influence the impact of HDs faced by OAs on depression risk (Hastings, George, Fillenbaum, Park, Burchett, \& Schmader, 2008; Kelley-Moore \& Ferraro, 2005; Koenig \& George, 1998; Potts, 1997; Turner \& Noh, 1988).

\section{Health Disparities and Depression}

Both biological/medical (e.g. chronic diseases, overall medical burden, vascular pathology, etc.) and psychosocial risk factors (e.g. life events, social support, loss, functional disability, etc.) have been found to be significantly associated with depression in OAs (Lawhorne, 2005). One association that has been consistent in the literature is the relationship between physical health and depression (Harman, Edlund, Forteny, \& Kallas, 2005). Prevalence rates of depression in OAs in outpatient medical settings range from 
$10 \%$ to greater than $30 \%$ (Kales \& Valenstien, 2002). Estimated prevalence rates are even higher in inpatient medical settings (12\%-45\%; Kales \& Valenstien, 2002). Eightyeight percent of OAs diagnosed with depression have at least one significant medical condition, and almost $50 \%$ have three or more (Lacro \& Jeste, 1994).

Additionally, OAs often have multiple chronic vascular conditions, which may lead to cerebrovascular changes in the brain over time. These brain changes may in turn contribute to depression (Alexopoulos, et. al., 1997). Although such vascular risk likely interacts with other medical and psychosocial risk factors of LLD, given the high prevalence of chronic medical conditions in OAs, vascular risk factors may be a unique mechanism that contributes to depression in OAs. In sum, physical health factors appear to be particularly relevant in the study of depression in OAs.

Poor physical health is a significant risk factor for developing LLD; and rural OAs are faced with a greater number of HD factors associated with poorer health status compared to their urban counterparts (Ebernhardt \& Pamuck, 2004). According to Probst and colleagues (2006), "rural residents are more likely than their urban peers to experience circumstances, conditions, and behaviors that challenge health and may increase the prevalence of depression (p. 653)." These researchers found that rural compared to urban residence was associated with a higher prevalence of depression and that the rural residents were more likely to have chronic medical diseases and poorer selfreported health status. The greater occurrence of HDs faced by rural OAs may increase the prevalence and risk for depression in rural OAs compared to their urban counterparts. Additionally, if rural OAs are faced with more chronic diseases that are vascular in nature (e.g. hypertension, heart disease, diabetes), there may also be a greater prevalence of 
vascular depression in rural compared to urban OAs. As vascular depression has been found to be particularly resistant to a number of common antidepressant treatments, knowledge of which groups of OAs are at increased risk for vascular depression is important (Fabre, Galinowski, Oppenheim, et al., 2004; Jorge, Moser, Acion, \& Robinson, 2008; Baldwin, Jefferies, Jackson, et al., 2004).

A number of the 24 studies referred to earlier investigate the relationship between health and depression in their rural samples (Blazer, George, Landerman, et al., 1985; Carruth \& Logan, 2002; Hauenstein \& Boyd, 1994; Linn \& Husaini, 1985; Muntaner \& Barnett, 2000; Probst, Laditka, Charity, et al., 2006; Sears, Danda, \& Evans, 1999). Six out of the seven general adult studies investigating such a relationship found the health variable researched to be significantly related to depression. Measures of health status utilized in these studies include: subjective rating of health (poor, fair, good, excellent), number of chronic conditions, perceived worsening of health status over the past year, and health related quality of life. In terms of rural OAs, Swenson and colleagues (2000) investigated the association between the number of chronic diseases (categorized as 0,1 , or $>1$ ) and depression. They found that this variable was significantly associated with depression in their Hispanic and non-Hispanic White OA sample.

It appears that poorer health status and greater medical burden are significantly associated with depression in rural communities. Given that rural residence is associated with a significantly greater number of HDs, such as a greater prevalence of chronic medical conditions and NHBs (e.g. smoking), compared to their urban counterparts, it is possible that these disparities may increase the prevalence and risk for depression in rural residents. Further, because OAs have more chronic illness and greater medical burden on 
average compared to younger adults (Eberhardt, et al., 2001), such HD factors may be even more salient in depression rates of rural OAs.

The current study contributes to the literature in that it is the first study to examine in depth the relationship between HDs and depression in both a rural and urban OA sample. The most common way researchers have studied the association between physical health and depression in the past was to investigate the association between individual health conditions or perceived health status and depression. However, there are many other variables that are important to investigate when looking at the association between HDs and depression. The current study not only investigated chronic health conditions, but also looked at the association between depression and a number of other individual HD variables, such as Negative health behaviors (e.g. smoking, drinking, physical inactivity), contact with outpatient healthcare providers, and cultural beliefs related to healthcare utilization, such as self-efficacy, negative attitudes towards doctors. Next, a description of the HDs examined in the current study is provided.

\section{Contact with Healthcare Provider}

One of the most commonly researched HDs in rural populations is limited access to healthcare. "Many, if not most, rural and frontier areas are federally designated health professions shortage areas or medically underserved areas (Stamm, Lambert, Piland, \& Speck, 2007, p. 300)." Across all regions in the US, rural populations have less access to healthcare than their urban and suburban counterparts (Eberhardt, et al., 2001). The supply of general internists and medical specialists decreases steadily as urbanization decreases (Eberhardt, et al., 2001). Rural residents, on average, have to travel longer 
distances to see a physician, which is an additional barrier to gaining access to care (Stamm, Lambert, Piland, \& Speck, 2007).

Although this study did not investigate access to care as commonly defined (i.e. an environmental variable representing the number of health care providers in a given area), the researchers investigated the participants' individual contact with healthcare providers. Healthcare utilization (HCU) was defined as the number of times a participant went to an outpatient primary care physician, clinic, or emergency department in the previous 12 months. Measuring contact with outpatient healthcare providers allowed for the investigation of individual differences in $\mathrm{HCU}$, versus investigating a general description of the environment in which one lives, i.e. number of physicians in a given area, which would be the same for all participants living in that location.

Due to the cultural emphasis on self-reliance in rural communities, (explained in more detail below), it was hypothesized that on average, OAs in rural locations likely have fewer contacts with outpatient care providers compared to their urban counterparts. This is due to the belief that rural residents put off healthcare more often and rely more heavily on self-care methods and use of informal care from family and friends for medical problems (Komiti, Judd, and Jackson, 2006). Contrary to such expectations, results from a previous analysis on the Duke EPESE baseline data indicate rural OAs in North Carolina attended more outpatient visits in the previous 12 months compared to the urban participants (Blazer, Landerman, Fillenbaum, \& Horner, 1995). It is important to note that this analysis dichotomized rural and urban, considering four out of the five counties equally rural, and did not distinguish between varying levels of rurality/urbanicity for the five counties represented in this dataset. In addition, the rural 
residents in this study sample reported significantly poorer self-rated health compared to the urban residents (Blazer, et. al., 1995), which may suggest the rural sample, as defined in Blazer and colleagues' analysis (1995), was sicker on average compared to the urban participants. Although this finding is contradictory to expectation, contact with healthcare providers was included in this study to further investigate the relationship between HCU and level of rurality utilizing a more sensitive measure of rurality, and to investigate the impact of $\mathrm{HCU}$ on depression by level of rurality and the potential moderating effects of social support and physical disability on the association between HCU and depression. The potential moderating effects of social support and physical disability will be discussed in more detail below.

\section{Disease Prevalence}

The greater prevalence of certain diseases and health conditions (e.g. heart disease, chronic obstructive pulmonary disease (COPD), diabetes, hypertension, and arthritis) represent a HD more prevalent in U.S. rural OA compared to urban OA samples.

Nationally, the death rate due to ischemic heart disease and COPD is higher for rural compared to urban residents (Eberhardt, et al., 2001; Ebernhardt \& Pamuk, 2004). The prevalence rate of diagnosed diabetes is highest in rural counties (Averill, 2005; Ebernhardt \& Pamuk, 2004). Prevalence rates for hypertension were significantly higher in nonmetropolitan versus metropolitan areas for men in the South (Ebernardt \& Pamuk, 2004). The prevalence rates of self-reported arthritis are also higher in nonmetropolitan than metropolitan communities (Ebernardt \& Pamuk, 2004). Rates of self-reported 
obesity are up to $28 \%$ higher in rural compared to urban communities (Ebernhardt \& Pamuk, 2004).

The higher rates of chronic diseases found in rural areas are significant given the negative outcomes associated with chronic illness. Eighteen percent of rural adults reported having chronic conditions that caused activity limitation compared with $13 \%$ of suburban adults (Ebernhardt \& Pamuk, 2004). Measures of health and well-being have shown that rural populations have poorer health status compared to their urban counterparts (Ebernhardt \& Pamuk, 2004). The prevalence of chronic disease conditions based on level of rurality was investigated in this study.

\section{Negative Health Behaviors}

According to Paringer, (1979) the higher prevalence of chronic health conditions in rural residents may be related to characteristics of their specific environment and lifestyles. A number of negative health behaviors (NHBs) occur more often in rural compared to urban areas. Three NHBs commonly discussed in the literature include: smoking, heavy alcohol consumption, and physical inactivity. All three of these behaviors were investigated in the current study.

Nationally, adults living in the smallest rural counties are most likely to smoke and those living in urban and suburban counties are least likely to smoke (Eberhardt, et al., 2001; Ebernhardt \& Pamuck, 2004). Rural smokers also tend to smoke more cigarettes per day than urban smokers (Hutcheson, Greiner, Ellerbeck, Jefferies, Mussulman, \& Casey, 2008). Rates of significant alcohol consumption (5 or more drinks per day) vary by urbanization level. A study investigating adults in Western U.S. states found that adults were more likely to be categorized as heavy drinkers if they lived in a 
rural area compared to urban regions (Eberhardt, et al., 2001). Physical inactivity during leisure time is most common for men and women in rural counties (Eberhardt, et al., 2001; Ebernhardt \& Pamuk, 2004). Comparisons based on geographic region found the proportion of the population physically inactive during leisure time was highest in rural counties in the South (Eberhardt, et al., 2001).

\section{Rural Culture and Attitudes towards Healthcare}

In a recent commentary, Klugman (2008) notes: "rural residents have a culture that is unique and different from their urban counterparts (p. 57)." This "rural" culture may impact how residents define health, and affect their help-seeking behaviors for medical conditions (Klugman, 2008). Negative attitudes towards physicians (NAs) may represent an important HD, as a person's beliefs regarding doctors may affect his or her health behaviors (e.g. engaging in preventive healthcare) and utilization of available healthcare resources. Likewise, individuals' self-efficacy beliefs regarding health (e.g. their belief they can take care of health problems on their own) may also affect HCU. To date, empirical research is lacking in this area (Klugman, 2008).

One cultural variable believed to be particularly salient in rural communities includes the importance of self-efficacy (Komiti, Judd, and Jackson, 2006). According to Klugman (2008), rural residents tend to value self-reliance and hardiness, culturally defined as taking care of oneself. Going to a healthcare provider is considered a last resort and help-seeking may be seen as a sign of personal weakness in rural residents (Klugman, 2008). In such cases, rural residents may prefer to either cope on their own or receive care from informal sources of help, such as family and friends (Komiti, Judd, and Jackson, 2006). Although these characteristics can be found in some individuals in all 
U.S. populations, these cultural characteristics are believed to be more prevalent and widespread in rural populations (Komiti, Judd, and Jackson, 2006). The possible emphasis on self-efficacy in rural communities may suggest rural residents have a greater sense of personal control over their health. This study investigated self-efficacy as one measure of culture.

Self-efficacy is particularly relevant to the study of the association between HDs and depression as studies have found self-efficacy and mastery to be significantly associated with health and well-being (Cairney, Corna, Wade, \& Streiner, 2007; Wolinsky, Wyrwich, Babu, Kroenke, \& Tierney, 2003). According to Ferreira and Sherman (2006), a sense of personal control over ones' life, experiences, and environment helps individuals cope effectively with disease. Low self-efficacy and perceived control, on the other hand, lowers one's immune system functioning (Ferreira $\&$ Sherman, 2006), which could lead to worsening health and increased need for medical care. A low sense of self-efficacy and control has been associated with increased visits to general practitioners and lower perceived health status (Cairney, et al., 2003). Personal control and self-efficacy beliefs have also been associated with lower estimations of one's own risk for developing a controllable negative health condition (Ruthig, Chipperfield, Bailis, \& Perry, 2008).

More negative attitudes towards doctors (NAs) is another cultural aspect of rural residence described in the literature that is relevant to the study of HDs and depression. For example, worries about lack of confidentiality maintained by physicians has been associated with more negative attitudes towards medical help-seeking in rural residents (Aderibigbe, Bloch, \& Pandurangi, 2008; Hoyt, Conger, Valde \& Weihs, 1997; Wrigley, 
Jackson, Judd, \& Komiti, 2005). NAs may decrease rural residents' seeking of formal medical care, especially preventative healthcare.

If such rural cultural attitudes regarding self-efficacy and NAs do exist, they may have the potential to influence rural residents' physical health over time via lack of healthcare prevention and timely treatment of health conditions. Failure to treat medical diseases and conditions in a timely and consistent manner can potentially lead to a greater number of and more severe chronic health conditions, and thus be considered an additional HD in this population.

In the current study, the association between cultural attitudes that may affect HCU and depression are investigated. Although cultural beliefs are often mentioned as barriers to $\mathrm{HCU}$ in rural residents, this hypothesis has not been previously tested empirically. More specifically, this study investigated whether 1) there are differences in self-efficacy and NAs by level of rurality, and 2) whether differences in self-efficacy and NAs are significantly associated with HCU.

The literature reviewed above suggests there is a greater prevalence of HDs faced by OAs in rural communities compared to their urban counterparts. The important question is whether or not the greater prevalence of HDs found in rural communities accounts for greater severity and rates of depression in rural compared to urban residents. Many of the HDs more prevalent in rural communities, (e.g. chronic health conditions and NHBs) have been consistently linked with depression, including obesity (Baumeister \& Harter, 2007; Dong, Sanchez, \& Price, 2004; Onyike, Crum, Lee, Lyketsos, \& Eaton, 2003; Simon, Von Korff, Saunders, et al., 2006), coronary heart disease (Carney \& Freedland, 2008; Goldston \& Baillie, 2008), COPD (Alexopoulos \& Latoussakis, 2004; 
Sirey, Raue, \& Alexopoulos, 2007; Stage, Middelboe, \& Pissenger, 2005), diabetes (Blazer, Moody-Ayers, Craft-Morgan, \& Burchett, 2002; Hu, Amoako, Gruber, \& Rossen, 2007), hypertension (Wei, Zeng, Chen, et al., 2004), cigarette smoking (Covey, Glassman, \& Stetner, 1998; Glassman, Helzer, Covey, et al., 1990), alcohol use (Grant \& Harford, 1995), and physical inactivity (Camacho, Roberts, Lazarus, Kaplan, \& Cohen, 1991; Farmer, Locke, Moscicki, Dannenberg, Larson, \& Radloff, 1988),.

HDs and their relationship to depression may be especially relevant to OAs. According to Lyness and colleagues (1996), "medical illness is the most consistently identified factor associated with the presence of late-life depression and is the most powerful predictor of poor depressive outcome (p. 198)." Many of the HDs present in rural populations are diseases often found to be more prevalent in OAs compared to the general population, including heart disease, diabetes, COPD, and hypertension (Alexopoulos, \& Latoussakis, 2004; Bosworth, Bartash, Olsen, \& Steffens, 2003; Eberhardt, et al., 2001; Fried, McNamara, Burke, \& Siscovivk, 1997; Hu, et al., 2007). Given the increased risk for chronic health conditions in OAs in general, combined with the greater prevalence of HDs in rural communities, rural OAs may represent a population that is particularly vulnerable to depression.

\section{Social Support and Physical Disability as Possible Moderating Variables on the Association between HDs and Depression}

To date, there are few studies investigating the role of moderators on the relationship between HDs and depression and level of rurality on depression. Relevant literature suggests that both social support and physical functioning may moderate these relationships. Social support may influence health in a number of ways, including having 
emotional support, informational support (regarding health), having positive (or negative) social influences (e.g. seeing others engage in positive health behaviors), and through access to material resources that have a direct influence on health (e.g. transportation assistance and financial assistance to access healthcare; Gorman \& Sivaganesan, 2007). In particular, one's perception of the availability of (or lack thereof) such social supports may be particularly relevant to the study of HDs and depression.

Literature discussing the relationship between social support and HCU reveals conflicting findings. A number of studies report individuals with low perceived social support and less access to informal social networks (i.e. family and friends) use healthcare services more often compared to individuals with high levels of perceived social support or greater access to a social network (Kouzis \& Eaton, 1998; Pilisuk, Boylan, \& Acredolo, 1987). Social support can be obtained by informal networks such as family and friends or by physicians (Kouzis \& Eaton, 1998). These findings suggest that when social support is unavailable through informal networks, patients may attempt to get support from their physicians by scheduling more frequent outpatient visits (Kouzis \& Eaton, 1998). Individuals with low perceived social support who use healthcare services more frequently may be particularly vulnerable to depression as both low perceived social support and high $\mathrm{HCU}$ are significantly associated with depression (George, Blazer, Hughes, \& Fowler, 1989; Koenig, Shelp, Goli, Cohen, \& Blazer, 1989). In contrast to the above findings, other researchers have found that persons with greater social support and greater levels of distress (including medical/physical distress) use medical services more frequently than individuals with less support and low distress. For example, Kouzis and Eaton (1998) found that individuals with high distress and 
greater social support were twice as likely to see a physician as the comparison group (low distress, less support); and that individuals with low distress and greater social support were $54 \%$ more likely to use healthcare services as the comparison group. Such findings "may be an example of social support encouraging appropriate use of health services among persons with an illness (Kouzis \& Eaton, 1998), p. 1307).” In other words, social support may appropriately increase healthcare use in some situations, through available resources to help one access care and encouragement to take care of oneself from family and friends. These findings lend themselves to a hypothesis of how social support may moderate the relationship between HCU and depression, and the relationship between HCU and level of rurality on depression. This hypothesis, in conjunction with the literature on rural culture and environments described above, suggests there may be a lower prevalence of depression in individuals with higher perceived social support and who have higher HCU, as these individuals not only have adequate perceived social support, but likely have better healthcare maintenance, more encouragement and support to maintain one's health, and greater perceived physical health.

Social support may also moderate the relationship between chronic diseases (overall medical burden) and depression. In a review of studies assessing the effect of social support on the course of chronic disease, a greater level of perceived social support was found to favorably affect disease course, including the outcomes of psychological adjustment and well-being (Pennix, Kriegsman, van Eijk, Boeke, \& Deeg, 1996). Fukukawa (2004) found that health problems were significantly related to depressive symptomotology in a sample of middle-aged Japanese adults, and that emotional support 
from family members significantly buffered this association. Such findings suggest that individuals with chronic diseases and greater perceived social support may have less depressive symptomotology compared to persons with chronic diseases and less social support.

In addition to a possible two-way interaction between HDs and social support on depression, this study also investigated the three-way interaction between HDs, perceived social support, and level of rurality on depression. However, to date, no studies were identified that investigated the moderating effects of social support on the association between rurality and depression. Kim and colleagues (2004) investigated these three variables in a different model, by investigating the moderating effects of rurality on the association between social support and depression in a Korean OA sample. These researchers found that lower social support (a composite measure of six individual items assessing different categories of social support) was most strongly associated with depression in OAs with lifetime rural residence.

Although no studies have investigated the moderating effects of social support on the HD-depression link in urban versus rural OAs, Gorman and Sivagansan (2007) investigated a mediating and moderating effect of social support on the association between SES and health factors (perceived health status and hypertension). No mediation effect was found; however, social support was found to be a significant moderator of the SES/health association, with substantial evidence suggesting that the measures of social integration buffered the negative effects of being in a low SES group. Overall, perceived social support may be particularly important for rural OAs because of the greater overall medical burden in rural residence and therefore need for healthcare 
access, health information, and emotional support from family and friends. In addition, previous studies have indicated its value as a potential moderating variable when examining the association between demographic characteristics and depression.

Physical disability is an additional variable that may moderate the relationship between HDs and depression, including $\mathrm{HCU}$ and overall medical burden, and the relationship between HDs and level of rurality on depression. According to Thomas (1999), it is difficult for individuals with disabilities to access primary healthcare services due to the physical barriers that result from their functional impairments. Further, the results of a survey conducted by Veltman and colleagues (2001) found a significant number of individuals with physical disabilities feel they experience difficulty accessing primary healthcare services. Physical disability and poorer health status are both significantly associated with depression (Koenig \& George, 1998), therefore, individuals with physical disability and less HCU (possibly due to difficulty accessing care) may be at the highest risk for depression. In addition, because chronic medical conditions are often associated with depression, adequate medical care for persons with a chronic disease becomes particularly important. If physical disability causes difficulty accessing care in persons with a chronic disease, physical impairment may also moderate the relationship between chronic diseases and depression. In this case, individuals reporting both the presence of chronic disease and physical impairment are likely the most vulnerable to depression. Likewise, in rural locations where access to care (and access to resources in general) is a common disparity, OAs with greater physical disability may have an even harder time accessing care and resources. Therefore greater physical 
disability may be particularly important for more rural OAs because of the increased difficulty accessing healthcare and resources.

Given the negative outcomes associated with depression, a greater understanding of the factors that lead to depression in differing OA populations is needed in order to develop more effective screening methods and prevention and treatment interventions. If HD variables are shown to account for greater depression severity in rural versus urban OAs, then utilizing a health disparity framework for depression in this population could inform research on prevention strategies, treatment, healthcare, and the development of more valid and clinically useful assessment and screening measures. Reducing HDs may not only increase the quality of life of rural residents, but may also reduce the health, social, and economic costs associated with both medical disease and depression. Therefore, it is imperative that the association between HDs and depression be investigated in U.S. rural and urban OAs.

\section{Research Questions and Hypotheses}

Based on the literature reviewed, the current study will address the following research questions and hypotheses:

1. The literature reviewed above suggests rural residents are faced with a greater number and severity of HD variables compared to their urban counterparts. It was hypothesized that rural OAs in the current sample will report a greater number and severity of HDs compared to the urban participants. Specifically, rural OAs, on average, will have a greater number of chronic health conditions, a greater number of NHBs, and less frequent HCU compared to more urban OAs. 
2. What is the relationship between rurality and depression? Do HD variables mediate the relationship between rurality and depression? It was hypothesized that in crosssectional analyses, level of rurality would be significantly related to depression, such that depression severity would increase as rurality increased. It was also hypothesized that a greater severity of HDs in more rural locations would account for greater depression severity, such that HDs would mediate the relationship between level of rurality and depression.

3. Are rural OAs at a greater risk for vascular depression compared to urban OAs? It was hypothesized that level of rurality would be significantly related to cumulative vascular risk (CVR), such that CVR would increase as rurality increased. Further, is was predicted that CVR would mediate the relationship between rurality and depression, similar to above, such that greater CVR in rural OAs would account for greater depression severity compared to urban OAs. This is based on research findings that rural residents have a higher prevalence of vascular disease conditions (e.g. heart disease, diabetes, hypertension) and NHBs associated with vascular disease (e.g. smoking) compared to urban OAs. The relationship between CVR and depression by level of rurality was looked at separately, as cerebrovascular disease resulting from vascular risk factors has been hypothesized to be a separate mechanism to the development of depression (Alexopoulos, et. al., 1997).

4. Does perceived social support moderate the relationship between HDs and depression and the relationship between rurality and HDs on depression? It was hypothesized that perceived social support would moderate the relationship between two HD variables: 1) less HCU and 2) greater overall medical burden, such that individuals with greater HDs 
and less perceived social support would have the highest risk for depression. Finally, it was hypothesized that a significant three-way interaction would exist, such that individuals in more rural counties, with greater levels of these two HDs, and less perceived social support would report the greatest level of depression.

5. Does physical disability moderate the relationship between HDs and depression and the relationship between rurality and HDs on depression? It was hypothesized that individuals reporting 1) less $\mathrm{HCU}$, and 2) greater overall medical burden, in conjunction with greater physical disability would be at the highest risk for depression. Finally, it was hypothesized that individuals in more rural locations, with greater levels of these two HD variables, and greater physical disability would report greater depression severity. 6. Literature discussing rural culture and HDs often states that rural residents emphasize self-efficacy, which may in turn relate to more self-care for medical problems and waiting longer periods of time to see a medical professional when sick (Klugman, 2008). Although this association is often mentioned, it has rarely been tested. Do rural OAs report greater levels of self-efficacy compared to urban OAs? And, is reporting greater levels of self-efficacy significantly associated with less HCU? It was hypothesized that rural OAs would report a greater mean level of self-efficacy compared to urban OAs and that higher self-efficacy scores would be associated with less HCU. Likewise, do rural OAs report more negative attitudes towards medical doctors (NAs)? And is having more NAs significantly related to less HCU? It was hypothesized that rural OAs would have more NAs and that having more NAs would be associated with less HCU.

7. Finally, are HDs more important to the development of incident depression over time in rural compared to urban OAs? The literature previously reviewed suggests rural OAs 
are faced with a number of HDs, including greater overall medical burden (more chronic health conditions), greater CVR, more NHBs, and possibly, less HCU. Therefore, it was hypothesized that rural OAs without significant depression at baseline would be at an increased risk for incident depression $(C E S-D \geq 9)$ at follow-up (3-years, 6-years, and 9years later) compared to their urban counterparts. Further, it was hypothesized that higher levels of the HDs investigated would mediate the association between level of rurality and incident depression. 


\section{Sample}

\section{METHODS}

This study used a prospective cohort design with four waves of data (baseline and three in-person follow-ups) from the Duke Established Populations for Epidemiologic Studies of the Elderly (EPESE): Piedmont Health Survey of the Elderly (Duke EPESE). The Piedmont Health Survey of the Elderly is a multiyear study sponsored by the National Institute on Aging. This data makes up one component of the Established Populations for Epidemiologic Studies of the Elderly (Cornoni-Huntlely, Blazer, Lafferty, Everett, Brock, \& Farmer, 1990). The EPESE project was created to investigate risk factors for chronic diseases and loss of functioning and to identify predictors of mortality, hospitalization, and placement in long-term care facilities (Cornoni-Huntley, Blazer, Service, \& Farmer, 1990). The EPESE was conducted at four geographic locations: East Boston, Massachusetts; New Haven, Connecticut; Iowa and Washington Counties, Iowa; and five counties in the north central Piedmont area of North Carolina. The data in the current study solely includes the North Carolina sample as only the Duke EPESE dataset was utilized. The Duke EPESE data is a particularly appropriate dataset to address the research questions investigated in the current study as it is comprised of a large sample size composed of residents living in five North Carolina counties that vary in their level of rurality (highly urbanized counties, suburban counties, and a rural county). This dataset also includes data representing the HD variables of interest in the current study. 
The North Carolina cohort was established in a 1986-1987 baseline survey, and includes non-institutionalized OAs age 65 or older residing in Durham, Warren, Franklin, Granville, and Vance counties in the Central Piedmont area of North Carolina. The data collected cover demographic characteristics (age, sex, race, income, education, marital status, number of children, employment status, etc.), social and physical functioning, chronic health conditions, health habits, self-reported use of healthcare and community services, and depression. A four-stage, stratified, probability sample was obtained with an oversampling of Black participants in order to generate a representative sample of the non-institutionalized North Carolina population aged 65 years or older. See Blazer, Burchett, Service, \& George (1991) for a more detailed explanation of the sampling procedures.

Baseline data collection took place during face-to-face interviews conducted in 1986 and 1987. Seven follow-up interviews were conducted over the course of the study: three additional in-person interviews and four short telephone interviews. The in-person waves were all conducted three years apart, with the final in-person wave collected in 1995-1996. Out of the 5,226 eligible sample members, 4,165 completed the baseline interview. Approximately $65 \%$ of all North Carolina study participants were female. Approximately $54 \%$ of the sample was (Non-Hispanic) Black, $45 \%$ was Non-Hispanic White, and less than $1 \%$ was of another racial background.

The five North Carolina counties included in this study are Durham, Franklin, Granville, Vance, and Warren, and were each contiguous to at least one other county in the catchment area. Forty-eight percent of the study sample is from Durham County, 13 percent is from Franklin, 13 percent is from Granville, 16 percent is from Vance, and 10 
percent is from Warren County (Cornoni-Huntley, et al., 1990). The sampling procedures were conducted to optimize both racial and rural/urban differences. Therefore, the current "sample was drawn in order to interview approximately equal numbers of subjects form urban and rural counties (Blazer, et al., 1991, p. M211).” However, rural and urban were originally defined by the U.S. Census Bureau definition, which only dichotomizes rural and urban status, and defines rural as a "place of residence with fewer than 2,500 inhabitants" (Blazer, Landerman, Fillenbaum, \& Horner, 1995). Durham County was considered the only primarily urban county, with $48 \%$ of the sample drawn from this county, and the four other counties were considered primarily rural, with the remaining $52 \%$ of the sample being drawn from these four counties combined. However, the Census definition utilized for the sampling procedures identifies only $63.2 \%, 65.2 \%$, and $89.2 \%$ of Vance, Granville, and Franklin Counties as "rural," respectively (Blazer, et al., 1995).

In the current study, rurality is based on the ERS rural/urban continuum codes for each of the 5 counties. These codes changed for Vance County and Warren County over the course of the study (see Table 2). At baseline, Durham and Franklin Counties were designated as code 2 (a county in a metro area of 250,000 to 1 million people). This code falls along the urban end of the rural/urban continuum. Granville County was a code 6 at baseline (a nonmetropolitan county with an urban population of 2,500-19,999 and adjacent to a metropolitan center). This code equates to what individuals might label a "suburban" location. At baseline, Vance County was a code 7 (a nonmetropolitan county with an urban population of 2,500 to 19,999 not adjacent to an urban center). Finally, Warren County was a code 9 (a nonmetropolitan county completely rural or less than 
2,500 urban population not adjacent to an urban center) at baseline. This is the most rural continuum code. Over the course of the study, Vance county moved from code 7 in 1983 to code 6 in 1993 to code 4 in 2003, and Warren County moved up one tier from code 9 in 1983 to code 8 in 1993 and 2003.

Although differences in the number of healthcare providers present in each county will not be investigated in the current study, data on such variables indicates environmentally-defined access to healthcare is as expected for each of the five counties based on level of rurality. During baseline collection, Durham County (the most urban of the five counties) had a healthcare delivery system that was "more extensive, complex and specialized than that locally available in the other four counties (Cornoni-Huntley, et al., 1990, p. 2)." This was true even after controlling for county size. Durham County had five hospitals. Franklin, Granville, and Vance Counties each had one general hospital, and Warren County did not have its own general hospital (this County's hospital was closed in 1985 due to low utilization). The distribution of nonfederal physicians varied greatly by county, which generally followed the basic pattern of fewer physicians as rurality increased. Durham County had 796 physicians, Franklin County had 11, Granville County had 48, Vance County had 28, and Warrant County had 6 (CornoniHuntley, et al., 1990).

The current analyses include only those participants with complete depression data at baseline. This excludes 169 individuals who either 1) did not answer all items on the CES-D ( $\mathrm{n}=8)$, or 2$)$ whose interviews were conducted by proxy informants $(\mathrm{n}=$ 161), as informants were not administered the depression measure. This results in a total sample size of 3,996 participants at baseline. The sample sizes for participants with 
complete depression data at in-person waves 2,3 , and 4 were 3,$003 ; 2,122$; and 1,399 respectively.

Measures

Background Variables

A number of sociodemographic variables were looked at by level of rurality including: gender, age, ethnicity, marital status, work status, education level, and annual household income based on level of rurality.

\section{Rurality}

Level of rurality was determined by the ERS rural/urban continuum codes. Baseline county data (1986-1987) was compared to the available 1983 coding system, which is available on the United States Department of Agriculture, Economic Research Services website (http://www.ers.usda.gov/Data/RuralUrbanContinuumCodes/). This coding system is a 9-tiered system and can be seen in Table 3. The code status for each of the five North Carolina counties can be seen in Table 2. Code classifications for the counties at follow-up waves were determined by the closest available code by year (1993 for in-person Wave 2; See Table 2). Whereas the 5 North Carolina counties fall into 4 ERS rural/urban continuum codes at baseline, they fall into 3 separate code levels at inperson Wave 2. The corresponding codes were used depending on the Wave of data utilized in the analysis (i.e. Wave 1 vs. Wave 2). The ERS rural/urban continuum codes represent a more detailed coding system which allowed the investigators to compare small, isolated, rural counties, to less rural counties close to an urban center. This, in turn allowed for the examination of the association between HDs and prevalence of 
depression in rural and urban OAs using a more accurate and variable measure of rurality.

\section{Depression}

Overall level of depressive symptoms was measured using a modified version of the Center for Epidemiologic Studies Depression Scale (CES-D). The CES-D was developed to help identify depressive symptoms in the general population and is the most widely used depression scale in studies with older adults (Black, Markides, \& Miller, 1998; Radloff, 1977). The CES-D is a 20 -item scale. Items on the original scale are measured on a four-point frequency scale, with total scores ranging from 0 to 60 , with higher scores indicating greater levels of depressive symptomatology. The modified version in the Duke EPESE study utilized a "yes/no" format for reporting the presence or absence of a symptom during the previous week. All 20 questions were verbatim from the original version of the questionnaire, and resulted in a total score ranging from $0-20$. Duke researchers previously conducted a number of analyses to ensure the revised instrument compared to the original scale in terms of its reliability and validity. Duke investigators used factor analysis to compare the Duke EPESE sample as a whole, and men, women, Blacks, and Non-Blacks separately, to those of the New Haven EPESE sample, who utilized the original CES-D measure. With few exceptions, the results were similar across the two depression measures (Blazer, Burchett, Service, \& George, 1991). The Duke researchers also utilized a regression line to compare the New Haven whole scale results to a dichotomized version of the New Haven data, which indicated that a cut-off score of $\geq 9$ is equivalent to the CES-D cut-off score of $\geq 16$ on the original scale representing prevalent depression (Blazer, et al., 1991; Black, et al., 1999). The original 
CES-D scale has been shown to be highly valid and reliable when used with community dwelling OAs (Beekman et al., 1999; Lewinsohn, Seeley, Roberts, \& Allen, 1997). A cut-score of $\geq 16$ on the original scale in community-dwelling OAs resulted in $100 \%$ sensitivity for major depression and $88 \%$ specificity (Beekman et al, 1999). Reliability analysis of the 20 modified CES-D items in the current sample resulted in a Cronbach's $\alpha$ $=0.82$.

$\underline{\text { Health Disparity Variables }}$

\section{Healthcare Utilization.}

Healthcare utilization (HCU) in the past 12 months was measured by the following interview item: "Not counting any care that you may have received while you were a bed patient in a hospital or nursing home, how many times did you receive care for a physical health problem from a health professional in an office, clinic, or emergency room in the past 12 months?" HCU was utilized as a continuous variable when investigating the relationship between HCU and the cultural variables (Self-efficacy and NAs). For chisquare, analysis of variance (ANOVA), and logistic regression analyses, in which HCU represented the independent, fixed-factor, $\mathrm{HCU}$ was recoded into 5 independent groups based on the number of times a participant received outpatient medical care in the past year $(0$ visits, $n=740 ; 1-3$ visits, $n=1382 ; 4-6$ visits, $n=913 ; 7-12$ visits, $n=548$; and $13+$ visits, $n=247$ ). These ranges equate to (1) individuals who did not utilize outpatient healthcare in the past year; (2) individuals who utilized outpatient healthcare at least once, but no more than once every four months on average in the past year; (3) individuals who utilized outpatient healthcare more than once every four months but no more than every two months on average in the past year; (4) individuals who utilized 
outpatient healthcare more that twice per month but no more than once per month on average in the past year; and (5) individuals who utilized outpatient services more than once per month on average in the past year. For analyses involving in-person Wave 2 data (e.g. culture analyses), the Wave $2 \mathrm{HCU}$ variable was utilized and was recoded into the same independent levels as the baseline HCU variable. The sample size for each group at Wave 2 is as follows: 0 visits, $(n=473) ; 1-3$ visits, $(n=1202) ; 4-6$ visits, $(n=$ $809)$; 7-12 visits, $(n=420)$; and $13+$ visits, $(n=187)$.

In order to determine the validity of this categorization, a one-way ANOVA was conducted to evaluate the relationship between HCU and depression. Chi-square analyses investigating the association between this categorical HCU variable and 2) overall medical burden, 3) physical functioning, and 4) perceived health status were also conducted. HCU was highly associated with mean depression score $(F(4,38)=47.484$, $\mathrm{p}<0.001$ ), such that mean depression score increased as HCU increased. HCU was also significantly associated with physical disability $\left(\chi^{2}(8)=212.42, p<0.001\right)$, overall medical burden $\left(\chi^{2}(12)=398.28, \mathrm{p}<0.001\right)$, and perceived health status $\left(\chi^{2}(12)=\right.$ 392.42, $\mathrm{p}<0.001)$. Healthcare utilization increased as physical disability increased, as overall medical burden (number of chronic health conditions) increased, and if a participant rated their perceived health status as poor or fair compared to good or excellent.

\section{Chronic Medical Conditions - Overall Medical Burden}

The presence or absence of chronic medical conditions was evaluated based on self-report questions. Study participants were asked whether a doctor had ever told them 
they had any of the following medical conditions: high blood pressure, cancer, diabetes, heart attack, stroke, and hip fracture. Respective verbatim prompts were as follows:

Has a doctor ever told you that you had (have) high blood pressure? Has a doctor ever told you that you had any cancer, malignancy, or (malignant) tumor of any type? Has a doctor ever told you that you had diabetes, sugar in your urine, or high blood sugar? Has a doctor ever told you that you had a heart attack, or coronary, or myocardial infarction, or coronary thrombosis, or coronary occlusion? Did a doctor ever tell you that you had a stroke or brain hemorrhage? Has a doctor ever told you that you had a broken or fractured hip?

It was also determined whether a participant met the National Institute of Health definition of obesity (body mass index; $\mathrm{BMI} \geq 30$ ) on the basis of his or her BMI. For all chronic medical conditions, the items were coded 1 if the condition was present and 0 if it was absent. A composite score of chronic medical conditions was formed, ranging from 0 to 7 , with higher scores indicating a greater number of chronic medical conditions (greater overall medical burden). This variable was then recoded into four groups based on the total number of chronic medical conditions each participant had at baseline $(0$ conditions, $n=965 ; 1$ condition, $n=1485 ; 2$ conditions, $n=982 ;$ and 3 or more conditions, $n=540$ ). These categories were statistically based on the percentage of participants in each category, with the goal of having a sufficient and relatively equivalent number of participants in each level. Although potential values ranged from 0-7 chronic disease conditions, less than $3 \%$ reported having four chronic conditions, less than $1 \%$ reported having five chronic conditions and six chronic conditions, and no participant endorsed all seven chronic conditions. Further, previous studies have also categorized composite scores created from the total number of disease conditions or health risk factors endorsed (Mast, MacNeill, \& Lichtenberg, 2004; Mast, Neufeld, 
MacNeill \& ichtenberg, 2004; Yochim, Kerkar, \& Lichtenberg, 2006; Zimmerman, Mast, Miles \& Markides, 2008).

\section{Cumulative Vascular Risk Index}

A similar composite score was created specifically for vascular risk conditions. These included the high blood pressure, diabetes, heart attack, stroke, and obesity variables listed above along with a smoking risk variable. The smoking risk variable was created utilizing two interview items: 1) "Do you smoke cigarettes regularly now?" And 2) "Did you ever smoke cigarettes regularly?" If a participant responded yes to either of these two items, they were coded as having smoking risk. Similar to above, the smoking item was scored 1 if the participant answered yes and 0 if he or she answered no. A composite measure of cumulative vascular risk (CVR) was formed, ranging from 0 to 6 , with higher scores indicating a greater number of vascular risk conditions present. This variable was then recoded into four groups: $0(n=576), 1(n=1,408), 2(n=1,201)$, and 3 or more (778) vascular risk conditions. Similar to the overall medical burden measure, these categories were statistically based on the percentage of participants in each category and are similar to categories utilized in previous studies (Mast, MacNeill, \& Lichtenberg, 2004; Mast, Neufeld, MacNeill \& ichtenberg, 2004; Yochim, Kerkar, \& Lichtenberg, 2006; Zimmerman, Mast, Miles \& Markides, 2008).

\section{Negative Health Behavior Index}

Literature discussing negative health behaviors (NHB) in the context of HDs includes: smoking, alcohol consumption, and physical inactivity. A dichotomized measure was created for each of these behaviors representing behavior risk versus no behavior risk. The items were coded 1 if the negative health behavior risk was present 
and 0 if it was not. These 3 items were then summed to create a composite measure of NHBs ranging from 0 to 3, with higher scores indicating a greater number of NHBs present. This variable was then recoded into three groups: 0 NHBs $(n=571), 1$ NHB ( $n$ $=1,581)$, and 2 or more NHBs $(n=789)$, based on sample size at each level. The manner in which these four variables have been dichotomized into risk/no risk is detailed below. Given that information on exercise (physical activity) was not collected until in-person Wave 2, all of the NHBs were created utilizing Wave 2 in-person data. In addition, all analyses utilizing the NHBs variable utilized corresponding Wave 2 variables (e.g. Wave 2 depression \& Wave 2 rural/urban continuum codes).

\section{Smoking}

The Duke EPESE dataset includes data on current and past smoking behaviors. The smoking risk variable was created utilizing two interview items: 1) "Do you smoke cigarettes regularly now?" And 2) "Did you ever smoke cigarettes regularly?" If a

participant responded "yes" to either of these two items he was coded 1 (smoke risk). All other participants were coded 0 . Self-reported smoking behavior has been successfully utilized in a number of studies and its validity has been verified with biomarkers, such as saliva cotinine (Stevens \& Munoz, 2004).

\section{Alcohol Use:}

The Duke EPESE data includes information on the consumption of beer, wine and liquor, including the frequency and quantity of alcohol consumed within the past month. According to the Center for Disease Control (CDC), the Dietary Guidelines for Americans defines moderate drinking as having no more than 1 drink per day for women and no more than 2 drinks per day for men. Heavy drinking is typically defined as 
having more than 1 alcoholic drink per day on average for women and more than 2 drinks per day on average for men (Alcohol and Public Health, 2008). The CDC defines binge drinking as a pattern of drinking that usually corresponds to 5 or more drinks on a single occasion for men or 4 or more drinks on a single occasion for women (Alcohol and Public Health, 2008). A dichotomous drinking risk measure was created based on these guidelines. Alcohol risk was coded 1 for men who consumed more than 2 drinks per day in the previous month (heavy drinking) or who drank 5 or more drinks on one occasion in the past month (binge drinking episode); and for women who consumed more than 1 drink per day in the previous month (heavy drinking) or who drank 4 or more drinks on one occasion in the past month (binge drinking episode). All other participants were coded 0 (no alcohol risk). Self-report items asking about the quantity and frequency of "usual" drinking patterns is a common measurement technique in epidemiological survey research (Hasin \& Carpenter, 1998), and self-report alcohol consumption questions have been found to be as valid as daily diary methods (Flegal, 1990).

\section{Exercise (Physical Inactivity)}

Exercise risk was measured by asking the participants: "In an average week do you do physical exercises?" Participants who answered "yes" were coded as 0; participants who answered "no" were coded as 1 . Self-reported engagement in exercise has been utilized in past gerontological research (Lindwall, Rennemark, Halling, Berglund, \& Hassmen, 2006). Research has indicated that participation in a regular exercise program can prevent and reduce a number of functional and health associated 
impairments in OAs (Tanaka, 2009). Regular exercise in the elderly has also been associated with decreased risk for depression (Lindwall, et. al., 2006).

\section{Culture Variables}

Negative Attitudes towards Medical Doctors

Perceived stigma and negative attitudes towards medical doctors (NAs) have been associated with rural residence (Aderibigbe, Bloch, \& Pandurangi, 2008; Hoyt, Conger, Valde \& Weihs, 1997; Wrigley, Jackson, Judd, \& Komiti, 2005). Therefore, attitudes towards medical doctors were investigated. The items assessing attitudes towards medical doctors in the Duke EPESE data are adapted from items contained in the Health Opinions Questionnaire of the Medical Access Study, a national telephone survey fielded by the National Opinion Research Center (NORC) at the University of Chicago (Howard, Bunch, Mundia, Konrad, Edwards, Amamoo, \& Jallah, 2006). Investigators have often used these items to describe constructs such as "attitudes toward doctors," but have not combined them into well validated scales (Howard, et al., 2006). In the Duke EPESE study, the original NORC questions were slightly altered "to reflect an explicit reference to older people, e.g., the original item: 'Doctors always treat their patients with respect' was changed to 'Doctors always treat their patients my age with respect' (Howard, et al., 2006, p. 2160)." Using a 4-point likert scale, participants were asked how much they agreed (strongly agree, agree, disagree, strongly disagree) with 10 statements about medical doctors. The items that make up this "negative attitudes towards doctors" scale can be found in Appendix C. Reliability analysis for the 10 items in the current sample resulted in a Cronbach's $\alpha=0.77$ 
A categorical NAs variable was created by utilizing a three-way split (i.e. approximately $1 / 3$ of subjects in each level), representing low (10-21), moderate (22-23), and high ( $>23$ ) levels of NAs. In an attempt to validate the categorization levels, a oneway ANOVA was conducted to investigate the relationship between the categorical NAs variable (3-levels) and depression (total CES-D score). Wave 2 depression score was used, as the items assessing NAs were first administered at the in-person, Wave 2, interview. The analysis revealed the categorical NAs variable is highly associated with depression $(\mathrm{F}(2,1,468)=25.73, \mathrm{p}<0.001)$, such that individuals with more NAs report higher depression scores on average $($ Low $=2.10$, Moderate $=3.11$, High $=3.72)$. Posthoc analyses revealed all three groups were significantly different from each other (Low vs. Moderate, $\mathrm{p}<0.001$, Low vs. High, $\mathrm{p}<0.001$, Moderate vs. High, $\mathrm{p}=0.025$ ). Self-Efficacy

Self-efficacy was measured by an adapted version of The Pearlin Mastery Scale (Pearlin \& Schooler, 1978). This scale is comprised of seven items that originally uses a 4-point scale ranging from strongly agree to strongly disagree. In the current study, participants were asked if they agreed or disagreed with the seven statements presented in this measure, which were verbatim to the original measure. These items were summed to create a total score ranging from $0-7$, higher scores indicating greater perceived selfefficacy. The Pearlin Mastery Scale contains items that assess for feelings of general self-efficacy and one's ability to solve problems through self-effort (Desocio, Kitzman, \& Cole, 2003). Five of the items elicit beliefs about self-efficacy to control current life circumstances and the remaining two items elicit beliefs about the ability to influence future possibilities (Desocio, Kitzman, \& Cole, 2003). The original Cronbach's alpha for 
the Pearlin Mastery Scale was .72 (Desocio, Kitzman, \& Cole, 2003). Reliability analysis of the modified seven items in the current sample resulted in a Cronbach's $\alpha=$ 0.67. The items that make up this measure can be found in Appendix D.

A categorical self-efficacy variable was created by utilizing a three-way split (i.e. approximately $1 / 3$ of subjects in each level), representing low (0-3), moderate (4-5), and high (6-7) levels of self-efficacy. In attempt to validate these categorization levels, a oneway ANOVA was conducted to investigate the relationship between the categorical selfefficacy variable (3-levels) and depression (total CES-D score). Wave 2 depression score was used, as the Pearlin Mastery Scale were first administered at the in-person, Wave 2, interview. The analysis revealed the categorical self-efficacy variable is highly associated with depression $(\mathrm{F}(2,2,392)=264.86, \mathrm{p}<0.001)$, such that individuals with less self-efficacy report higher depression scores on average (Low $=4.86$, Moderate $=$ 2.48 , High $=1.19$ ). Post-hoc analyses revealed all three groups were significantly different from each other at the $\mathrm{p}<0.001$ level.

$\underline{\text { Potential Moderator Variables }}$

Activities of Daily Living (Physical Disability)

The six-item Index of Activities of Daily Living (ADL) scale developed by Katz and colleagues (1963) was utilized to assess one's ability to independently engage in the following activities of daily living: bathing, dressing, toileting, transferring, continence, and feeding. Reliability and validity of this ADL scale is well established (Kane \& Kane, 1981). Two additional items, grooming and walking across a small room, were also included. These two items come from the work of Branch and colleagues (Foley, Fillenbaum, \& Service, 1990). For each ADL other than incontinence, study participants 
were asked the following question: "Was there any time in the past 12 months in which you needed help from some person or from some equipment or device to do [the activity]." A "yes/no" answer format was used. A score of 1 indicates the person had difficulty performing the ADL and a score of 0 indicates no difficulty in performing the ADL independently. Difficulty holding one's urine or bowels was determined using the following two interview questions: "How often do you have difficulty holding your urine until you can get to a toilet; never, hardly ever, some of the time, most of the time, or all of the time?" And: "In the past few months have you ever lost control of your bowels (when you didn't want to)?" If the participant reported having difficulty holding their urine at least some of the time or ever losing control of his or her bowels, they received a score of 1 for incontinence. All other participants received a score of 0 . Total scores ranged from 0 to 8, with higher scores indicating greater levels of ADL limitation or impairment in physical functioning. Reliability analysis for the eight ADL items in the current sample resulted in a Cronbach's $\alpha=0.80$.

A categorical physical disability variable was created, which included 3 levels: 0 reported ADL limitations $(\mathrm{n}=2,197), 1$ reported ADL limitation $(\mathrm{n}=1,251)$, and 2 or more reported $\mathrm{ADL}$ limitations $(\mathrm{n}=524)$. In order to validate this categorization a oneway analysis of variance was conducted to investigate the association of this categorical physical disability variable (3-levels) and depression (total CES-D score). The results indicate the categorical physical disability variable is highly associated with mean depression score $(F(2,3,969)=213.73, p<0.001)$, such that individuals who reported more physical disability received higher depression scores on average ( 0 ADL limitations (2.34), 1 ADL limitation (3.64), 2+ ADL limitations (3.94). Post hoc analyses revealed 
all three groups were significantly different from each other at the $p<0.001$ level. In addition, chi-square analyses investigating the association between the categorical physical disability variables and both overall medical burden (4-levels) and perceived physical health (4-levels) were conducted. The categorical physical disability variable was significantly associated with both overall medical burden $\left(\chi^{2}(6)=211.09, p<0.001\right)$ and perceived physical health $\left(\chi^{2}(6)=402.31, \mathrm{p}<0.001\right)$, such that individuals with a greater number of ADL impairments reported more chronic medical conditions and poorer perceived physical health.

\section{Social Support}

Two items from the Duke Social Support Index (DSSI) assessing level of perceived social support were collected in the Duke EPESE study (Hastings, et al., 2008). These items were combined using factor analytic procedures to create a composite measure of perceived social support. More support is coded as high and less support is coded as low (Landerman, 1998). The perceived social support index consists of the following two study items: 1) "In times of trouble, can you count on at least some of your family and friends most of the time, some of the time, or hardy ever?" And 2) "Can you talk about your deepest problems with at least some of your family and friends most of the time, some of the time, or hardly ever?" Cronbach's alpha was only .55 for this composite measure. Although this value is below the rule of thumb minimum of .70, Landerman, et al. (1989), developer of the composite indexes, suggests this is likely due to the small number of constituent items making up this composite measure and encourages the use of the composite perceived social support measure over the individual study items. Literature suggests that an individual's subjective perception of social 
support is the most strongly related social support variable to mental health outcomes (Landerman, George, Campbell, \& Blazer, 1989).

A categorical variable was created for perceived social support. The range of possible scores was from 2-6 on this measure. A score of 2 and 3 were combined due to the small sample size at each level and due to the fact that scores of 2 and 3 represent individuals who reported particularly low levels of perceived social support. The categorical perceived social support measure was broken down into 4 separate levels: score of 2-3 (low perceived social support; $\mathbf{n}=173$ ), score of 4 (moderate perceived social support, $n=455$ ), score of 5 (high perceived social support $n=619$ ), and a score of 6 (very high perceived social support $n=2,728$ ), with the majority of participants reporting the highest level of perceived social support in the current sample. In order to validate this categorization, a one-way ANOVA was conducted to investigate the association between the categorical perceived social support measure and depression (total CES-D score). The results indicate the categorical perceived social support measure is highly associated with depression $(\mathrm{F}(3,3,971)=51.14, \mathrm{p}<0.001)$, such that individuals who reported less perceived social support received higher depression scores on average (level (mean depression score): $2-3$ (5.24), 4 (4.26), 5 (3.67), 6 (2.84)). Post hoc analyses revealed all groups were statistically significant from each other. Imputed variables

Five variables utilized in this study were previously imputed by Duke EPESE investigators, and the use of the imputed variables are encouraged over their non-imputed counterparts by the Duke EPESE investigators. These variables include: annual income, education (in years), marital status (currently married vs. not currently married), BMI, 
and perceived social support (constructed composite measure). For measures with 5\% or more missing data, imputed versions were created using stochastic regression techniques, in which imputed values were a predicted score obtained by regressing the variables to be imputed on correlates of the same wave. For the composites with at least $2 \%$ but less than 5\% missing data, a regression-based y-hat was substituted for missing values. The exception was BMI, in which height and weight imputed values were imputed from respondent's non-missing height and weight values from a different wave whenever possible (EPESE Composite Measures, 1999; Missing Value Imputations, 1992).

\section{Statistical Power and Significance Level}

Given the large number of analyses that were conducted in the current study, the problem of an inflated type-I error rate needed to be addressed. Although Bonferronicorrection is commonly used to address inflated family-wise error rate when multiple analyses are being conducted, this correction is too conservative for the current study, due to the large number of anlyses. While it is important to lower alpha to account for the large number of computations in this study, reducing the significance level too conservatively can lead to Type II error. Therefore, a more liberal approach was utilized and all analyses were compared to a more moderate alpha level, $\alpha<0.01$.

Using G-Power 3.0.3, given the Wave 4 in-person sample size of 1,399, the power to detect a small effect size $(\mathrm{d}=0.02)$ with alpha set at 0.01 for Analysis of Variance is 0.88 . With a very small effect size $\left(f^{2}=0.01\right)$, alpha set at 0.01 , and 12 predictor variables, power for the smallest sample size (Wave 4 sample $=1,399$ ) is equal to 0.88 for linear regression. 


\section{Statistical Analyses - Descriptive Analyses}

All analyses were carried out using SPSS 18.0. Differences in demographic variables were examined across the four baseline levels of rurality including participant age, gender, education, income, race, marital status, and work status via one-way analysis of variance (ANOVA) and chi-square analyses.

One-way ANOVAs were utilized to investigate the association between the demographic variables included above and mean depression score. For these analyses, age was categorized into age-ranges commonly used in older-adult research (3-levels), which are as follows: 65 - $74(\mathrm{n}=2499), 75-84(\mathrm{n}=1230)$, and $85+(\mathrm{n}=267)$. Income and education were categorized utilizing quartiles (i.e. approximately $25 \%$ of the sample in each level). The four levels for education were as follows: 0 - 5 years $(n=965) ; 6-8$ years $(n=1132) ; 9-11$ years $(n=984)$; and $12-17+$ years $(n=915)$. The four levels for income were as follows: $\$ 999.50$ $\$ 3,499.50(\mathrm{n}=792) ; \$ 4,499.50-\$ 5,999.50(\mathrm{n}=1473) ; \$ 8,499.50-\$ 12,499.50(\mathrm{n}=977)$; and $\$ 17,499.50$ - $\$ 44,529.80(n=754)$. Finally, the race variable utilized was recoded to include self-identified Non-Hispanic White $(n=1819)$ and Black $(n=2153)$ participants only, as less than $1.0 \%(n=24)$ of the sample identified themselves as another race.

Finally, chi-square analyses were utilized to investigate the bivariate associations among the main $\mathrm{HD}$ variables (overall medical burden, cumulative vascular risk, healthcare utilization, \& negative health behaviors), culture variables (self-efficacy and negative attitudes towards doctors), and hypothesized moderator variables (perceived social support \& physical disability) in this study. 
Statistical Analyses - Hypothesis Testing

The first research hypothesis examined the severity of HDs, including overall medical burden (i.e. number of chronic health conditions; 4 levels), NHBs (i.e. total number of negative health risk behaviors endorsed; 3-levels), and HCU (i.e. number of outpatient medical visits in the past year; 5-levels) by level of rurality (i.e. ERS rural/urban county continuum codes; 4 levels at Wave 1 and 3 levels at Wave 2). In order to determine if $\mathrm{HD}$ variables are significantly more severe in rural compared to urban counties, chi-square analyses were conducted.

The second research hypothesis initially predicted greater depression in more rural counties, and that greater depression severity in more rural counties would be accounted for by a greater number and severity of HDs in more rural locations. However, tests of mediation effects could not be conducted given the non-significant differences in the severity of depression and rates of prevalent depression across county codes. Tests of moderation effects were also conducted in order to test the possibility that HDs and level of rurality interact on depression severity, to determine if having more severe HDs and living in more rural counties leads to greater depression severity. Factorial ANOVAs were conducted for overall medical burden, HCU, and NHBs, controlling for significant demographic variables. Total CES-D score was the dependent variable of interest. The categorical HD variable and level of rurality (county code) represented the fixed, independent, factors. The two-way interaction effect of the factorial ANOVAs allowed for the evaluation of the interaction between the HD variable and level of rurality on depression. 
The third study hypothesis first examined the association between cumulative vascular risk (CVR; 4-levels) and level of rurality (4-levels). Next, this hypothesis predicted that there would be greater depression in more rural counties and that CVR would mediate the relationship between level of rurality and depression. A test of mediation was not conducted, however, given the lack of differences in depression severity and rates of prevalent depression by county code. However, similar to above, a test of moderation was also conducted for CVR to determine if CVR and level of rurality interact on depression severity. First, a chi-square analysis was conducted to determine if significant differences in CVR exist by county code (4 levels at Wave 1). Second, a factorial ANOVA was conducted in order to determine if a significant interaction existed between CVR and level of rurality on depression. CVR (4 levels) and level of rurality (County Code, 4 levels) represented the fixed, independent, factors, and the total CES-D score represented the dependent variable.

The fourth study hypothesis examined the two-way interaction between perceived social support and two HD variables, (greater overall medical burden and less HCU,) on depression. It was hypothesized that individuals with greater levels of the HD variables and lower perceived social support would report the greatest depression severity. This hypothesis also investigated the three-way interaction between perceived social support, the HD variable of interest, and level of rurality (county code) on depression. It was hypothesized that individuals with greater levels of the HD variables, lower perceived social support, and who were living in more rural counties would report the greatest depression severity. Overall medical burden and HCU were the HD variables investigated in this hypothesis based on previous research suggesting perceived social 
support may moderate the relationship between these two HD variables and depression (Fukukawa, 2004; Kim, Stewart, Shin, Yoon, \& Lee, 2004; Kouzis \& Eaton, 1998; Pennix, Kriegsman, van Eijk, Boeke, \& Deeg, 1996). Factorial ANOVA was utilized to examine the two-way and three-way interactions between these variables, after controlling for significant demographic characteristics. Total CES-D score was the dependent variable. Perceived social support, the HD variable, and county code represent the fixed, independent factors.

The fifth study hypothesis examined the two-way interactions between physical disability (number of physical limitations) and the HD variables (overall medical burden and HCU) on depression. It was hypothesized that individuals with greater levels of HDs and greater physical disability would report the highest depression scores. This question also investigated the three-way interaction between physical disability, the HD variables, and level of rurality (county code) on depression. It was hypothesized that individuals with greater levels of HDs, greater physical disability, and who live in more rural counties would report the highest depression scores. Similar to above, overall medical burden and $\mathrm{HCU}$ were the two HD variables investigated in this hypothesis based on previous research suggesting physical disability may moderate the relationships between these HDs and depression (Thomas, 1999; Veltman, Stewart, Tardif, \& Branigan, 2001). Factorial ANOVA was utilized to examine the two-way and three-way interactions between these variables, after controlling for demographic characteristics. Total CES-D score was the dependent variable. Physical disability, the HD variables, and county code represent the fixed factors. 
The sixth study hypothesis addressed whether there are differences in cultural attitudes (self-efficacy and negative attitudes towards doctors (NAs)) between rural and urban older adults, and whether self-efficacy and NAs affect HCU. First, in order to determine if differences exist in mean levels of reported self-efficacy and NAs by level of rurality, one-way ANOVAs were conducted, with rurality as the independent variable with 3 levels (at Wave 2) and level of reported self-efficacy (3 levels) and NAs (3-levels) as the dependent variables. As stated previously, the self-efficacy and NAs measures were first administered at Wave 2, therefore only Wave 2 data, including the corresponding ERS 1993 (closest year available to 1987 Wave 2 data) rural/urban continuum codes, were utilized. Second, the relationships between 1) self-efficacy and HCU, and 2) NAs and HCU were evaluated. Bivariate correlations were utilized to test the hypothesis that higher levels of self-efficacy and more NAs would be related to less HCU. The continuous HCU, self-efficacy, and NAs variables were utilized in this analysis.

The final study hypothesis predicted there would be greater rates of incident depression, in individuals without significant depressive symptomotology at baseline, as rurality increased. It was further predicted that the HD variables (overall medical burden, CVR, HCU, and NHBs) would mediate this relationship, such that a greater severity of HDs in more rural counties would account for increased risk for significant depressive symptomotology at follow-up. First, chi-square analyses were conducted to determine if significant differences existed in the risk for incident depression (CES-D $\geq 9$ ) at 3-, 6-, and 9-year follow-up waves by level of rurality. These analyses were conducted on those participants with low levels of depression at baseline (CES-D $\leq 5$ ). A CES-D score $\leq 5$ 
was utilized as the cut off for minimal depressive symptoms because a change of at least four points on the CES-D represents reliable change using the reliable change index (SD $=3.42 ; \alpha=8.82 ;$ reliable change index $=3.98$ for baseline CES-D). Therefore, those participants who score greater than 9 at follow-up would have demonstrated a clinically significant change in depressive symptoms. As no significant differences in the rates of indecent depression were found by level of rurality at the in-person follow-up interviews, longitudinal mediation tests were not conducted. 


\section{RESULTS}

\section{Demographics of sample}

The demographic characteristics of the participants are presented in Table 4. No significant differences were observed for gender $\left(\chi^{2}(3)=3.33, p=0.34\right)$ and marital status $\left(\chi^{2}(3)=2.81, p=0.42\right)$ by urban/rural continuum code. The majority of the sample was female (65.1\%) and $38.6 \%$ were married. Mean age significantly differed by level of rurality $(\mathrm{F}(3,3992)=3.34, \mathrm{p}=0.02)$, with the significant difference occurring between county codes $7(72.83$ years) and $9(73.94$ years, $p=0.046)$. Code 9 (the most rural code) had a significantly greater prevalence of Black residents (73.51\%) compared to the three other county codes (range $49.12 \%-57.12 \% ; \chi^{2}(2)=79.59, \mathrm{p}<0.001$ ). Residents in code 2 (the most urban county), had significantly higher education ( $\mathrm{F}$ (3, $3992)=11.63, \mathrm{p}<0.001)$ on average $(8.84$ years $)$ compared to residents in codes 6,7 , and 9 (7.91 years to 8.21 years). Average annual income was significantly higher ( $\mathrm{F}(3$, $3992)=33.72, p<0.001)$ for residents in code 2 compared to the three other county codes. Residents in code 7 also had a significantly higher average annual income compared to code 9 residents $(\mathrm{p}=0.035)$. A greater percentage of residents in codes 2 $(13.55 \%)$ and $6(13.09 \%)$ were still currently working compared to residents in codes 7 $(9.08 \%)$ and $9\left(8.66 \% ; \chi^{2}(3)=14.83, p=0.002\right)$. Mean depression score (modified CES-D; $F(3,3992)=1.39, p=0.24)$ and the percentage of residents with prevalent depression (CES-D score $\geq 9 ; \chi^{2}(3)=2.15, p=0.54$ ) did not differ significantly between 
the four county codes. For the sample as a whole, the average modified CES-D score was 3.24 (out of 20 possible points) with a standard deviation of 3.42 ; and $9.6 \%$ of subjects scored above the cut-score on the CES-D suggesting prevalent depression. In his 2003 review, Blazer found that the prevalence range for clinically significant depressive symptoms among community-dwelling OAs is approximately $8 \%-16 \%$. The $9.6 \%$ of participants reporting clinically significant depressive symptoms in the current sample (CES-D $\geq 9$ ) falls on the low end of this range. Total depression score in the current study was not normally distributed and there was a relative floor effect of mean depression score in this sample (Figure 1). Approximately one fourth of the sample endorsed no CES-D items (23.4\%), and more than half of the sample (54.4\%) endorsed less than 2 CES-D items.

The association between the demographic variables listed above and depression (mean CES-D score) are summarized in Table 5. The results of these analyses indicate that on average Black participants had significantly higher depression scores than NonHispanic White participants. Women had significantly higher depression scores on average compared to men. Participants who were currently married had significantly lower depression scores compared to participants who were not married. Participants who were currently working had significantly lower depression scores on average compared to individuals who were not working. Mean depression scores were higher in older groups, in lower income groups, and in lower education groups.

Bivariate relationships among the main study variables are presented in Appendix E. Frequency tables for the main study variables for the sample as a whole are summarized in Appendix F. 


\section{Hypothesis 1 Analysis}

Hypothesis 1 predicted that HDs (greater overall medical burden, less HCU, and more NHBs) would significantly relate to level of rurality, such that a greater severity of these HD variables would be present as rurality increased. Table 6 summarizes the chisquare analyses examining the associations between level of rurality and $\mathrm{HCU}$, overall medical burden, and NHBs. Non-significant associations were found between 1) overall medical burden and rurality, and 2) NHBs and rurality, indicating these two HD variables do not differ by level of rurality in this sample. Table 6 provides the percent of participants at each level of the HD for the total sample by county code, and provides the mean and standard deviation for each variable for the total sample and each county code individually.

A significant association was found between rurality and $\mathrm{HCU}(\mathrm{p}<0.001)$. Follow-up post-hoc analyses are summarized in Table 7. Contrary to the initial hypothesis, the results indicated county code 2 (the most urban county) was significantly different from county code 9 (most rural county, $\mathrm{p}<0.01$ ), such that a higher percentage of code 2 residents were more likely to be low healthcare utilizers ( $0-3$ visits in the past year) compared to code 9 ; whereas code 9 residents were more likely to be more moderate to high healthcare utilizers (4-12 visits in the past year) compared to code 2 . Follow-up analyses were conducted to better understand these utilization differences between county codes 2 and 9, particularly since the findings were not consistent with the initial hypothesis. 


\section{Follow-up analyses}

Chi-square analysis revealed a significant difference in the usual place of healthcare services utilized ( 6 levels) by level of rurality $\left(\chi^{2}(15)=396.29, p<0.001\right)$. The six response categories for place of usual outpatient healthcare include: going nowhere, no usual place, private practice (solo group, clinic, private office in hospital), public clinic (including urgent or emergency care public clinic), outpatient other (VA, public, private, walk-in hospital), or other (including: ER VA, ER other hospital, employee health, other). Individuals in county code 9 were much more likely to utilize public clinics compared to individuals in code $2(35.7 \%$ vs. $11.4 \%)$ for their usual care, whereas individuals in code 2 were more likely to use private practice offices compared to code 9 (63.3\% vs. $52.4 \%)$, which represents a significant differences between codes 2 and $9\left(\chi^{2}(5)=193.33, p<0.001\right)$. In fact, individuals in code 9 were more likely to use public clinics compared to the other three county codes combined $\left(\chi^{2}(1)=190.14, p<\right.$ 0.001 ; Table 8$)$.

Moreover, participants who utilized public clinics went to the doctors more often $\left(\mathrm{HCU} ; \chi^{2}(4)=40.31, \mathrm{p}<0.001\right)$ and had more chronic medical conditions $\left(\chi^{2}(3)=\right.$ 43.26, $\mathrm{p}<0.001$ ) compared to individuals who used private practice offices to receive their usual healthcare. In addition, participants who utilized public clinics were more likely to be very dissatisfied with their healthcare compared to participants utilizing private practice offices (5.5\% vs. $3.8 \%$ ), and less likely to report being very satisfied with their healthcare compared to participants utilizing private practice offices $(24.7 \%$ vs. $38.5 \% ; \chi^{2}(3)=35.66, p<0.001$; Table 9). The satisfaction with healthcare variable consisted of four levels: very satisfied, satisfied, dissatisfied, and very dissatisfied. 
Although code 9 has a greater prevalence of high healthcare utilizers compared to code 2 , when investigating satisfaction with healthcare by county code in general, residents in code 9 were less satisfied with their healthcare services compared to code 2 residents $\left(\chi^{2}(9)=172.67, p<0.001\right)$. Ten and $1 / 2$ percent of participants in code 9 report being very dissatisfied with their healthcare, compared to $3.1 \%$ of residents in code 2 ; and only $21.3 \%$ of code 9 residents report being very satisfied with their healthcare services, compared to $42.2 \%$ of residents in code 2 . Table 10 summarizes differences is satisfaction with healthcare by all four county codes.

In addition, although code 9 had a significantly greater number of high healthcare utilizers compared to code 2 , code 9 also had significantly more residents who reported "going nowhere" for usual outpatient healthcare services in the past year $(4.2 \%)$ compared to county codes 2,6 , and $7\left(1.8 \% ; \chi^{2}(1)=10.39, p=0.001\right.$; Table 8$)$. In general, participants who reported "going nowhere" for regular healthcare services were also significantly more likely to report no healthcare visits in the past year on the HCU variable compared to individuals utilizing a regular place of healthcare $\left(\chi^{2}(4)=258.99, p\right.$ $<0.001 ; 88.9 \%$ vs. $17.8 \%$ ). Residents in county code 9 who reported "going nowhere" for healthcare service in the past year were significantly healthier than residents who reported utilizing regular healthcare services at least once in the past year $\left(\chi^{2}(1)=13.19\right.$, $\mathrm{p}=0.004)$, such that they were significantly more likely to report no chronic health conditions (52.9\% vs. $20.5 \%)$ and significantly less likely to endorse 2 or more chronic health conditions ( $5.9 \%$ vs. $41.8 \%)$.

Taken together, it appears there are more individuals in code 9 (the most rural code) who are actually underutilizing regular outpatient healthcare services compared to 
the more urban county codes $(2,6, \& 7)$, which is what was initially hypothesized. However, given the greater variability in HCU patterns and health status in county code 9 residents, the low utilizers of usual outpatient healthcare get averaged with the high prevalence of residents in code 9 who are more moderate to high healthcare utilizers. Follow-up analyses were conducted to investigate the role of race, income, and education on $\mathrm{HCU}$, as these three variables differed by county code and have been associated with HCU in previous studies (Thorson \& Powell, 2000). In terms of race (2levels, Black and Non-Hispanic White), HCU patterns significantly differed between Black and Non-Hispanic White residents $\left(\chi^{2}(4)=14.18, \mathrm{p}<0.01\right)$. Moreover, HCU patterns of Black and Non-Hispanic White participants differed by county code. There was no difference in HCU between Black and Non-Hispanic White participants in code 9 $\left(\chi^{2}(4)=1.32, p=0.86\right)$, but Black residents in code 2 utilized healthcare services more often in the previous year than Non-Hispanic White participants in code 2, a finding which approached significance $\left(\chi^{2}(4)=11.25, p=0.02\right)$. A similar pattern occurred for the association between race and overall medical burden. Black participants reported a greater number of chronic medical conditions than Non-Hispanic White participants when investigating the sample as a whole $\left(\chi^{2}(3)=30.05, \mathrm{p}<0.001\right)$. However, there was no difference in the number of chronic medical conditions between Blacks and NonHispanic Whites in code $9\left(\chi^{2}(3)=3.89, p=0.27\right)$, whereas in code 2, Black participants reported significantly more chronic health conditions compared to the Non-Hispanic White residents $\left(\chi^{2}(3)=19.88, p<0.001\right)$, which may explain the greater rates of $\mathrm{HCU}$ for Black participants in code 2. In terms of satisfaction with healthcare services for the sample as a whole, Black residents were less satisfied compared to Non-Hispanic White 
residents $\left(\chi^{2}(3)=68.41, p<0.001\right)$. However, this association differed by county code, such that there was no difference in satisfaction with healthcare between Black and NonHispanic White residents in code $9\left(\chi^{2}(3)=5.58, p=0.13\right)$, whereas Black residents in code 2 were less likely to be very satisfied with their healthcare compared to the NonHispanic White residents $\left(\chi^{2}(3)=39.53, p<0.001\right)$. The results of the above analyses are summarized in Table 11.

To tie these findings together, a three-way loglinear analysis was conducted to determine if a significant interaction existed between race, county code, and usual place of healthcare (private practice offices and public clinics). The three-way loglinear analysis produced a final model that retained all effects. The likelihood ratio for this model was $\chi^{2}(0)=0, p=1.00$, which indicates the highest-order interaction (Race $\mathrm{x}$ Code $x$ Usual Place of Healthcare) was significant $\left(\chi^{2}(15)=41.75, p<0.001\right)$. Table 12 provides the percentage of Black and Non-Hispanic White residents by county code and usual place of healthcare. Both Non-Hispanic White and Black residents in county code 9 were much more likely to utilize a public clinics compared to Non-Hispanic White and Black participant in the other three counties. In addition, a greater percentage of NonHispanic White residents utilized private practice offices across all four county codes compared to Black resident, and a greater percentage of Black residents utilized public clinics across all four county codes compared to Non-Hispanic White participants, a finding which may further support the presence if racial HDs.

In terms of income, when looking at the study sample as a whole, participants in lower annual income groups utilized healthcare services more frequently than individuals in higher annual income groups $\left(\chi^{2}(12)=37.86, \mathrm{p}<0.001\right)$. However, a similar pattern 
to race was observed for income, in that the association between income and $\mathrm{HCU}$ differed by county code. HCU patterns did not differ by annual income in code $9\left(\chi^{2}(12)\right.$ $=14.01, p=0.30$ ), whereas greater HCU was significantly associated with lower annual income groups in code $2\left(\chi^{2}(12)=31.64, p<0.01\right)$. Likewise, lower income groups in Code 2 reported significantly more chronic medical conditions (greater overall medical burden) compared to higher income groups $\left(\chi^{2}(9)=61.89, p<0.001\right)$, whereas there was no difference in overall medical burden between income groups in code $9\left(\chi^{2}(9)=10.57\right.$, $p=0.31)$. Place of healthcare differed by income $\left(\chi^{2}(3)=115.52, p<0.001\right)$, such that participants in lower annual income groups were significantly more likely to utilize public clinics. However, a three-way loglinear analysis revealed no interaction between income, place of usual healthcare, and county code $\left(\chi^{2}(9)=12.42, p=0.14\right)$. Further, as was the case for race, income was significantly associated with satisfaction with care, such that satisfaction levels were higher for higher annual income groups $\left(\chi^{2}(9)=\right.$ $122.19, \mathrm{p}<0.001)$.

There is a body of literature suggesting race may represent a proxy variable for income (socioeconomic status, SES). This does not appear to be the case in the current sample, however, given that Black participants had significantly less income than NonHispanic White participants in codes $2\left(\chi^{2}(3)=286.30, p<0.001\right)$ and $9\left(\chi^{2}(3)=59.73\right.$, $\mathrm{p}<0.001$ ) alike, yet significant differences between Black and Non-Hispanic White participants on the HD variables discussed above only occur in code 2 .

In terms of education, the association between education and $\mathrm{HCU}$ approached significance $\left(\chi^{2}(12)=23.98, p=0.02\right)$, such that participants in less education groups utilized more outpatient healthcare services in the previous year, although this association 
did not differ by county code $\left(\right.$ code $2: \chi^{2}(12)=21.74, p=0.04$; code $9: \chi^{2}(12)=19.09, p$ $=0.09$ ).

Although race, income, and place of usual outpatient healthcare services were not originally hypothesized to influence physical health and HCU patterns across levels of rurality, these factors appear to play an important role in understanding the lack of support for hypothesis 1 .

\section{Hypothesis 2 Analysis}

Hypothesis 2 predicted the HDs (overall medical burden, HCU, and NHBs) investigated in the current study would mediate the relationship between rurality and depression. However, a mediation effect could not be investigated because depression severity did not differ by county code (as detailed above). Analyses were also conducted to determine whether the $\mathrm{HD}$ variables investigated in this study moderate the relationship between level of rurality and depression, hypothesizing that individuals in the more rural counties and with more severe levels of the HD variables would have a greater level of depression on average. More specifically, it was hypothesized that a significant two-way interaction would exist between county code and 1) overall medical burden, 2) HCU, and 3) NHBs on depression after controlling for age, race, income, education, and work status, such that the individuals in more rural counties reporting higher levels of the HD variables would report greater depression severity.

The main effects of the factorial ANOVA revealed that greater overall medical burden $(\mathrm{F}(3,3950)=23.98, \mathrm{p}<0.001)$ and greater $\mathrm{HCU}(\mathrm{F}(4,3804)=23.01, \mathrm{p}<0.001)$ were both highly associated with a greater total depression score. Mean depression score 
increased as the number of chronic medical conditions increased $(0$ conditions $=2.57 ; 1$ condition $=3.05 ; 2$ conditions $=3.58$; and $3+$ conditions $=4.36)$ and as the number of outpatient healthcare visits in the last year increased $(0$ visits $=2.64 ; 1-3$ visits $=2.70 ; 4$ 6 visits $=3.36 ; 7-12$ visits $=3.90 ;$ and $>12$ visits $=4.93$ ). However, the NHB variable was not significantly associated with mean depression score $(F(2,2796)=0.13, p=$ $0.88)$.

Overall, there were no significant interactions between level of rurality and the three HD variables on depression, which indicates the impact of these HDs on depression did not depend on level of rurality in this sample. Figures $2-4$ display the mean depression score by level of the HD variable (overall medical burden, HCU, and NHBs respectively) for each county code.

As stated earlier, it was hypothesized that less HCU would be related to depression in more rural locations, as not seeking preventative healthcare or adequately treating medical condition when needed could lead to poorer health status, which is associated with depression. Contrary to this hypothesis, the analysis above indicated that, in fact, more $\mathrm{HCU}$ is related to greater depression severity.

As a follow-up, linear regression was utilized to investigate the hypothesis that overall medical burden would mediate the relationship between HCU and depression, as having more chronic medical conditions could lead to more $\mathrm{HCU}$ due to increased need for formal medical care. An initial linear regression was conducted to provide an estimate of the bivariate association between $\mathrm{HCU}$ and depression (CES-D score), after controlling for demographic variables in Block 1 (age, race, income, education, and work status). HCU was significantly associated with depression severity $(F(6,3822)=62.62$, 
$\mathrm{p}<0.001 ; \beta=0.20$ ). A second linear regression was then conducted to investigate the potential mediating effect of overall medical burden. Overall medical burden was entered into Block 2 and $\mathrm{HCU}$ was entered into Block 3. The change in HCU was small, and it remained a significant predictor of depression $(\mathrm{F}(7,3798)=61.70, \mathrm{p}<0.001 ; \beta=$ 0.16), indicating overall medical burden did not mediate the relationship between $\mathrm{HCU}$ and depression in this sample.

\section{Hypothesis 3 Analysis}

Hypothesis 3 investigated cumulative vascular risk (CVR) as a separate HD variable, as vascular disease has been described as a separate mechanism in the development of late-life depression, and literature suggests rural OAs report a greater number of VRFs (e.g. smoke risk, obesity, chronic vascular disease conditions, etc.) compared to their urban counterparts (Alexopoulos, et al., 1997). To begin, it was predicted that significant differences in the prevalence of CVR (4 levels) would exists by level of rurality (4 levels), such that residents in more rural counties would report a greater number of VRFs compared to urban OAs. A chi-square analysis revealed nonsignificant differences in the number of VRFs reported by level of rurality $\left(\chi^{2}(9)=9.68\right.$, $p=0.38 ;$ Table 6).

Next, it was hypothesized that CVR would mediate the relationship between level of rurality and depression. However, a mediation effect could not be investigated because depression severity did not differ by county code (as detailed above). Analyses were conducted to determine if CVR would moderate the relationship between level of rurality and depression, hypothesizing that individuals in the more rural counties and with 
a greater number of VRFs would report the highest depression scores. A factorial ANOVA was conducted to evaluate the two-way interaction between CVR and rurality on depression, after controlling for age, race, income, education, and work status. The main effect between CVR and depression revealed CVR was highly associated with depression severity $(\mathrm{F}(3,3941)=15.78, \mathrm{p}<0.001)$, such that mean depression scores increased as the number of vascular risk factors increased $(0 \mathrm{VRFs}=2.69 ; 1 \mathrm{VRF}=3.01$; $2 \mathrm{VRFs}=3.21 ; 3+\mathrm{VRFs}=4.11)$. The two-way interaction was not significant $(\mathrm{F}(9$, $3941)=0.87, p=0.55)$, which indicates that the impact of CVR on depression did not depend on level of rurality in this sample (Figure 5).

\section{Follow-up Analyses}

Follow-up analyses were conducted to better understand the non-significant association between rurality and depression and the non-significant mediation and moderation effects between level of rurality and the HD variables on depression. To begin, given income and education were both significantly related to depression severity and significantly differed by county code, three-way loglinear analyses were conducted to determine if income or education affected rates of prevalent depression (CES-D $\geq 9$ ) by county code. Prevalent depression rates were investigated due to the observed floor effect of mean depression scores across the entire sample (i.e. the mean CES-D score was

relatively low across all four county codes). Neither income $\left(\chi^{2}(9)=8.84, \mathrm{p}=0.45\right)$, nor education $\left(\chi^{2}(9)=16.40, p=0.06\right)$ significantly interacted with level of rurality and prevalent depression.

Next, given the significant difference in race by county code, and that Black participants reported more depressive symptoms (mean CES-D score $=3.41$ ) on average 
compared to Non-Hispanic White participants (mean CES-D score $=3.04 ; \mathrm{F}(18,3,953)$ $=3.38, \mathrm{p}<0.001$ ), follow-up analyses were conducted to determine if race affected rates of depression by county code. In order to determine if a significant interaction existed between race, county code, and prevalent depression (CES-D score $\geq 9$ ), a three-way loglinear analysis was conducted. The three-way loglinear analysis produced a final model that retained all effects. The likelihood ratio for this model was $\chi^{2}(7)=9.95, p=$ 0.19, which indicates the highest-order interaction (Race x Code x Prevalent Depression interaction), was significant at the $p<0.05$ level $\left(\chi^{2}(3)=7.62, p=0.046\right)$. Prevalent depression rates increased for Non-Hispanic White participants and decreased for Black residents as rurality increased (Table 13). The results of this analysis are displayed in Figure 6. Although race, was not originally hypothesized to influence rates of prevalent depression across levels of rurality, race appears to play an important role in understanding the lack of support for hypothesis 2 . Combined with findings from hypothesis 1 , racial disparities in terms of physical health and depression may be especially present for Black OAs living in more urban locations.

\section{Hypothesis 4}

Hypothesis 4 predicted that perceived social support would moderate the relationship between overall medical burden and depression, such that individuals with lower levels of perceived social support and more chronic health conditions would report the greatest depression severity. It was also predicted that perceived social support would moderate the relationship between $\mathrm{HCU}$ and depression, such that individuals with lower levels of perceived social support and less $\mathrm{HCU}$ would report the greatest levels of 
depression severity. Finally, it was predicted that a significant three-way interaction would exist, such that perceived social support would significantly interact with the HD variable and level of rurality, such that individuals in more rural counties and lower levels of perceived social support and greater levels of the HD variable would report the greatest level of depression. Although higher HCU was found to be associated with greater depression severity in this sample, it was still predicted that lower levels of perceived social support combined with low HCU would lead to greater depression, hypothesizing that individuals who feel they have a lack of support to help them access healthcare services, and who are unable to get to needed/wanted healthcare appointments (i.e. lowering their level of $\mathrm{HCU}$ ), would be the group at the highest risk for depression.

Factorial ANOVAs were conducted to test these hypotheses, after controlling for demographic variables, and are summarized in Table 14 (overall medical burden) and Table 15 (HCU). Results of both factorial ANOVAs revealed the association between perceived social support and depression (main effect) was highly significant $(\mathrm{p}<0.001)$, such that mean depression score increased as perceived social support decreased. The main effects for $\mathrm{HCU}$ and overall medical burden remained significant as in previous analyses, with greater $\mathrm{HCU}$ and overall medical burden being associated with greater depression severity. However, no significant interactions (two-way or three-way) were observed in either analysis, indicating that although perceived social support was highly related to depression, it did not interact with the HD variables or with level of rurality on depression.

Given the significant interaction between race, county code, and rates of prevalent depression detailed above, a three-way loglinear analysis was conducted between 
perceived social support, race, and prevalent depression, in order to help clarify the greater rates of prevalent depression in Black OAs as rurality decreased and in NonHispanic White OAs as rurality increased. The need for such analyses has been stressed by previous research (Brondolo, ver Halen, Pencille, Beatty, \& Contrada, 2009). The analysis was not significant $\left(\chi^{2}(9)=16.88, p=0.051\right)$, although approached significance at the $\mathrm{P}<0.05$ level. Descriptively, however, the highest percentage of Black residents with low perceived social support occurred in code 2, compared to the three other codes, whereas the highest percentage of Non-Hispanic White residents with low perceived social support occurred in code 9 (Table 16), a finding which parallels the patterns of prevalent depression rates in rural and urban Black and Non-Hispanic White OAs.

\section{Hypothesis 5 Analysis}

Hypothesis 5 predicted physical disability would also moderate the relationship between overall medical burden and depression, such that participants with greater physical disability and more chronic health conditions would report greater depression. It was also predicted that physical disability would moderate the relationship between $\mathrm{HCU}$ and depression, such that participants with greater physical disability and less HCU would report greater depression. Similar to perceived social support, it was further hypothesized that a significant three-way interaction would exists between physical disability, the HD variables and level of rurality on depression, such that individuals in more rural counties with greater physical disability and greater levels of the HD variables would report the greatest depression severity. Factorial ANOVAs were utilized to 
investigate these two-way and three-way interactions, after controlling for demographic variables.

The factorial ANOVAs are summarized in Table 17 (overall medical burden) and Table 18 (HCU). Results of both factorial ANOVAs revealed the association between physical disability and depression (main effect) was highly significant $(\mathrm{p}<0.001)$, such that mean depression score increased as physical disability increased. Again, the main effects for HCU and overall medical burden remained significant. No significant interactions (two-way or three-way) were observed in either analysis, indicating that although physical disability was highly related to depression, it did not interact with the HD variables or with level of rurality on depression.

Hypothesis 6 Analysis

Hypothesis 6 predicted that self-efficacy and negative attitudes towards doctors (NAs) would differ significantly by level of rurality (3 levels at Wave 2). Specifically, it was hypothesized that rural OAs would report higher levels of self-efficacy and more NAs. A one-way ANOVA revealed NAs was significantly associated with rurality $(\mathrm{p}<$ $.001)$. As expected, follow-up post hoc analyses revealed codes 2 and 6 were both significantly different from code $8(\mathrm{p}<0.001)$, such that residents in code 8 (most rural code) reported more NAs compared to individuals in codes 2 and 6 (most urban codes). The association between rurality and self-efficacy approached significance $(p=0.017)$. However, post-hoc analyses revealed that self-efficacy was slightly higher in code 2 versus code 8 residents, $(p=0.028)$, which is contrary to what was initially predicted. The results of the one-way ANOVAs are summarized in Table 19. 
Second, it was predicted that self-efficacy and NAs would be significantly related to $\mathrm{HCU}$ (Wave 2), such that greater self-efficacy and more NAs would be associated with less HCU. A significant correlation was observed between self-efficacy and HCU $(r=-$ $0.10, \mathrm{p}<0.001$ ) such that greater self-efficacy was associated with less HCU, as predicted. NAs, however, were not significantly associated with $\mathrm{HCU}(\mathrm{r}=0.03, \mathrm{p}=$ 0.34), indicating that having more negative attitudes towards doctors did not affect frequency of healthcare utilization in this sample.

A follow-up chi-square analysis was conducted to determine if self-efficacy was associated with overall medical burden. This follow-up analysis was conducted to help answer the question: do individuals with more self-efficacy utilize outpatient healthcare services less frequently than individuals with less self-efficacy because they choose to take care of their medical problems on their own (versus seeking formal medical care); or, do individuals with greater self-efficacy have few chronic medical conditions and therefore utilize healthcare services less frequently? It is possible that OAs with greater self-efficacy engage in more preventative care behaviors and positive health behaviors, which leads to fewer chronic medical conditions, and therefore less HCU. Results of a chi-square analysis indicate individuals with higher self-efficacy scores had significantly fewer chronic health conditions $\left(\chi^{2}(6)=40.11, p<0.001\right.$; Table 20).

Based on this finding, linear regression was utilized to investigate the hypothesis that overall medical burden would mediate the relationship between self-efficacy and $\mathrm{HCU}$, as engaging in preventative and positive health behaviors could lead to better health status (fewer chronic medical conditions) and less HCU. An initial linear regression was conducted to provide an estimate of the bivariate association between self- 
efficacy and $\mathrm{HCU}$, after controlling for demographic variables in Block 1 (age, race, income, education, and work status). As expected, self-efficacy was significantly associated with $\mathrm{HCU}(\mathrm{F}(6,2308)=10.30, \mathrm{p}<0.001 ; \beta=-0.70)$, such that individuals with greater self-efficacy utilized healthcare services less often. A second linear regression was then conducted to investigate the potential mediating effect of overall medical burden. Overall medical burden was entered into Block 2 and self-efficacy was entered into Block 3. The change in self-efficacy was small, and it remained a significant predictor of depression $(\mathrm{F}(7,2294)=29.11, \mathrm{p}<0.001 ; \beta=-0.57)$, indicating overall medical burden did not mediate the relationship between self-efficacy and HCU in this sample.

\section{Hypothesis 7 Analysis}

Hypothesis 7 predicted that the risk for incident depression would be greater as rurality increased in participants without significant depressive symptomotology at baseline (CES-D score $\leq 5$ ). Further, it was predicated that the HD variables in this study would mediate the relationship between level of rurality and incident depression. Initial chi-square analyses revealed non-significant differences in the rates of incident depression by level of rurality in all three in-person follow-up interviews (3-, 6-, and 9years later), for participants without significant depressive symptomotology at baseline. Thus, a mediation effect could not be investigated. The results of these analyses are summarized in Table 21 . Given the cross-sectional analyses investigating potential interactions between the HD variables and level of rurality on depression were not significant, longitudinal analyses investigating such interactions were not conducted. 


\section{DISCUSSION}

This study explored the association between HDs and depression in a sample of rural and urban OAs, and examined whether HDs contribute to our understanding of how rurality impacts depression severity and prevalence of depression. The initial overarching hypothesis suggested that rural OAs would be faced with a greater severity of HDs compared to their urban counterparts, including engaging in more negative health behaviors (NHBs), having more chronic health conditions and vascular risk factors, and underutilizing healthcare services (for preventative care and treatment of medical issues), and as a result, would have greater depression.

The overarching hypothesis was not supported by the current study. Level of rurality was not, in and of itself, significantly associated with depression severity or rates of prevalent or incident depression (CES-D $\geq 9$ ). Further, other than HCU, the HD variables investigated in the current study did not differ by level of rurality; and although HCU was significantly associated with rurality, contrary to the initial prediction, rural OAs utilized outpatient services more frequently in the past year compared to their urban counterparts. In addition, the HD variables investigated did not significantly mediate or moderate the association between level of rurality and depression in this sample, suggesting that overall medical burden, CVR, HCU, and NHBs, do not significantly influence the relationship between level of rurality and depression in the current sample. 
One implication of the current results, however, is that there is important variability within the county codes that may help to explain the non-significant differences in depression and HDs, across the four county codes. In other words, the global ERS continuum codes do not appear to capture the variability that exists within each county code. For example, the results suggest two distinct rural groups exist in county code 9 in terms of physical health status and HCU patterns. One group consisted of moderate to high healthcare utilizers who were more likely to report having two or more chronic health conditions (i.e. were sicker). The second group of code 9 rural OAs were more likely to report "going nowhere" for regular outpatient healthcare over the past year compared to code 2 residents, and were healthier than the high healthcare utilizers in code 9 (i.e. more likely to report having no chronic medical conditions). However, this greater percentage of code 9 rural OAs who were low healthcare utilizers was averaged in with the moderate to high healthcare utilizers, suggesting that when differences in $\mathrm{HCU}$ patterns were investigated by county residence alone, important variability within each county was lost.

In addition, follow-up analyses suggest that although in general, the rural/urban county code variable alone was not directly associated with HDs and depression, individual characteristics (e.g. race, income, and place of usual outpatient healthcare services) may help clarify the relationship between level of rurality and both HDs and depression. For example, results suggest that rates of prevalent depression differ by race across rural and urban status, such that rates of prevalent depression increase as rurality increases for Non-Hispanic White participants, but decreases as rurality increases for Black participants. This suggests that investigating difference by level of rurality alone, 
even if broken down into more sensitive groups, may not be sufficient when investigating rural/urban differences in HDs and factors that affect rates and severity of depression. Other individual characteristics (e.g. race) need to be investigated in conjunction with rural/urban status.

Finally, support was also found for the notion that cultural variables, including self-efficacy and negative attitudes towards doctors (NAs) differ by rural and urban status, and that self-efficacy significantly impacts HCU patterns.

Seven main research hypotheses were addressed in the current study with the broad aims of describing the relationship between HDs, level of rurality, and depression. The study findings overall address three main research questions, which will be discussed in more detail below. These include: 1) Is rural residence associated with HDs; 2) Do rural elders have greater depression and do HD's account for this; and 3) What is the value of a more specific rural/urban definition? Overall, given the initial overarching hypothesis was not supported, the main goal of the following discussion is to try to understand this non-significant finding and what factors may be critical to better understating the relationship between level of rurality, physical health variables (HDs), and depression.

Study Question 1: Is rural residence associated with HD's including poorer health (greater overall medical burden), health care utilization, and cultural health beliefs that may affect health behaviors and physical health status? 


\section{Health status:}

In general, rural older adults in the current sample did not report a greater number of chronic medical conditions, VRFs, or NHBs, a finding inconsistent with previous research (Ebernhardt \& Pamuk, 2004). The non-significant association between these three HD variables and rurality in the current study could be related to the fact that the majority of participants in this study were in relatively good health. Only $13.6 \%$ of participants reported having three or more chronic health conditions (out of seven possible conditions), $19.6 \%$ reported having three or more VRFs (out of a possible six VRFs), and only $13.2 \%$ reported having two or more ADL limitations (out of eight possible limitations). It is possible that the limited variability in the current communitybased sample in terms of health status was insufficient to investigate differences in depression and health across level of rurality. It is also possible the current HD measures were not sensitive enough to capture differences in health across county codes. For example, the overall health burden measure included only seven health conditions. Other studies have investigated differences in rural and urban elders utilizing a more thorough list of health conditions (e.g. Parks \& Neutens, 1987, who utilized a checklist of over 40 medical conditions).

The same limitation applies to the NHBs variable, which included only three health risk behaviors (smoke risk, alcohol risk, an exercise risk), and had limited variability (e.g. less than $1 \%$ of the total sample engaged in all three NHBs measured, with $3 / 4$ engaging in one or less ). Further, only $1.2 \%$ of the entire sample met criteria for alcohol risk. Taken together, the variability on this measure was very low in this sample. With low variability within measures, which was particularly true of NHBs, it is difficult 
to demonstrate covariance with other measures, such as level of rurality. Although previous studies have utilized composite measures of NHBs in order to investigate the combined effect of the included health risk behaviors (Dressler, Oths, \& Gravlee, 2005), a more valid composite measure assessing NHB risk is clearly needed.

\section{Healthcare utilization:}

Although, HCU was found to be significantly associated with level of rurality, contrary to the initial hypothesis, residents in county code 9 utilized more outpatient healthcare services compared to resident in county code 2. Although this finding is consistent with Blazer and colleagues' (1995) finding utilizing a dichotomous measure of rurality in the current Duke EPESE sample, this finding is inconsistent with existing literature suggesting rural residents put off healthcare more often and rely more heavily on self-care methods and use of informal care from family and friends for medical problems (Komiti, Judd, and Jackson, 2006). Thorson \& Powell (2000), also found no difference between their urban and rural OA sample when investigating the frequency of visits to one's primary care provider; although found that urban OAs were significantly more likely to report receiving an annual physical exam compared to the rural participants. Follow-up analyses attempted to explain this finding and are discussed in detail below. In particular, it appears that where urban and rural elders seek their usual outpatient healthcare, in addition to their physical health status, may be important sources of variability that could help clarify the lack of support for the initial hypothesis.

As stated earlier, follow-up analyses revealed that although, overall, code 9 had the highest percentage of moderate to high healthcare utilizers, code 9 also had a 
significantly greater percentage of participants who reported "going nowhere" for usual place of outpatient healthcare compared to code 2 residents. It appears there were two distinct groups of rural OAs residing in county code 9 in terms of physical health status and frequency of outpatient care. One group consisted of moderate to high healthcare utilizers who reported having a usual place of healthcare (e.g. a private practice or public clinic) and were more likely to report having two or more chronic health conditions (i.e. were sicker). This group of high healthcare utilizers in code 9 likely resulted in the finding that rural OAs utilize outpatient healthcare services significantly more frequently compared to code 2 residents.

However, there was a second group of code 9 rural OAs who were more likely to report "going nowhere" for regular outpatient healthcare over the past year compared to code 2 residents, and were healthier than the high healthcare utilizers in code 9 (i.e. more likely to report having no chronic medical conditions). It is possible that healthier (i.e. no chronic health conditions) rural OAs are less likely to utilize preventative healthcare services (e.g. regular check-up and screens), which may over time lead to the development of preventable chronic medical conditions or an increase in the severity of a medical condition, such as heart disease and diabetes, which would then shift them into the other group of over-utilizers. An active decision by rural OAs not to use formal healthcare services would support the original hypothesis.

It appears to be the case, however, that at some point, when chronic medical conditions develop and become more severe, there becomes a need for formal medical care and more frequent $\mathrm{HCU}$. Greater $\mathrm{HCU}$ in individuals with a greater number of chronic medical conditions has been supported in previous research. Thorson and Powell 
(2000) investigated the association between health beliefs and HCU. The health beliefs investigated consisted of three main categories: predisposing factors (e.g. health beliefs), enabling factors (e.g. health knowledge \& financial resources), and need factors (e.g. number of chronic health conditions). The need factor (e.g. having more chronic medical conditions) was consistently found to be the most important factor influencing healthcare utilization.

Support for this prediction would suggest a slightly modified version of the initial overarching hypothesis, in which rural OAs initially utilize healthcare services less often, (particularly preventative and maintenance healthcare services), until unavoidable due to the severity of their medical conditions, which eventually leads to the need for more frequent formal healthcare. Such a hypothesis fits with previous research linking cultural characteristics of the rural sample (e.g. "stoicism") to delayed health-seeking, which has been attributed to a general pattern of later diagnosis, more advanced disease, and poorer prognosis of residents in rural areas (Howat, Veitch, \& Cairns, 2006).

In terms of one's usual place of outpatient healthcare, more specifically, residents in code 9 were much more likely to utilize public clinics compared to code 2 , whereas individuals in county code 2 were much more likely to utilize private practice offices compared to code 9 for usual place of outpatient healthcare. The general assumption in the literature has been that rural OAs are faced with a distinct disadvantage in terms of healthcare access, and therefore, health outcomes (Thorson \& Powell, 2000). In the current rural sample (Warren County, NC), it appears access to care was not a significant barrier faced by the older residents. Initial reports on the Duke EPESE data indicated that participants who reported having a usual place of healthcare tended to report that their 
provider's office was in the same county as their county of residence (Balzer, et al., 1995). Taken together, the availability and frequent use of public clinics indicates the rural OAs in this sample likely had access to healthcare services if and when needed.

Although access to care and availability of healthcare providers was not a focus of the current study, it may represent an important variable making the current rural sample different compared to rural samples used in previous research studies. The availability of healthcare providers, in a relatively close radius to one's place of residence, are factors frequently found to be related to HCU (i.e. the "distance decay effect," Acury, Gesler, Preisser, et al., 2005). The apparent availability of public healthcare providers in this rural sample may indicate access to healthcare was not a present HD in Warren County, North Carolina. Although easy access to healthcare cannot explain the greater rates of HCU in the current rural versus urban sample, it may suggest that this commonly cited barrier to $\mathrm{HCU}$ in rural locations was not present in the current sample.

However, although code 9 residents clearly had access to healthcare services, their more frequent use of public clinics, may suggest a HD in terms of available healthcare options. In other words, although there was a group of code 9 residents who went to more frequent outpatient visits in the past year compared to residents in code 2 , they were more likely to use public clinics and were more likely to be dissatisfied with the healthcare they received compared to code 2 residents. Further, study participants in general, who used public clinics for outpatient healthcare, reported more chronic medical conditions and went to the doctor more often, which may suggest differences in the quality and effectiveness of care administered at public clinics versus private practice offices. Taken together, the results may suggest disparities in terms of the quality of 
health services available to rural OAs and in terms of the quantity of healthcare options. Future studies are needed to clarify this picture and to investigate the effects of limited healthcare options and dissatisfaction with healthcare on physical and mental health outcomes.

In addition to usual place of outpatient healthcare, it appears race and income represent additional sources of variability that could help clarify the lack of support for the initial hypothesis that rural OAs would report less HCU. For example, the current study found that Black and Non-Hispanic White residents differed in their HCU patterns by county code. Race and income have been found to influence HCU patterns in previous research (Thorson \& Powell, 2000).

The impact of race on $\mathrm{HCU}$ patterns by county code was also investigated as the population of Black and Non-Hispanic White residents differed significantly by county code in the current sample. Further, although the current study was interested in HDs based on residence (i.e. rural/urban status), race and ethnicity are among the most commonly used variables in public health research investigating differences in HDs based on population groups (Dressler, Oths, \& Gravlee, 2005). However, Dressler and colleagues (2005) have warned that "race" tends "to be used uncritically and without definition (p. 234)," and that it is the responsibility of the researchers to investigate the causal mechanisms of racial differences. Possible mechanisms for the racial differences in the current study are explored below, which need to be investigated in future studies. In terms of the definition of "race" in the current study, participants were asked: "What is your race," with the following answer options: Black (non-Hispanic); White (nonHispanic); Hispanic; Aleutain, Eskino, or American Indian; Asian or Pacific Islander; and 
Other. Therefore, race was defined as the individual participant's self-reported racial group he or she identified with most. However, due to the very small proportion of participants that identified with a racial category other than Black or Non-Hispanic White, the current analyses focus only on differences between the self-identified Black and Non-Hispanic White participants.

As noted above, when investigating the sample as a whole, code 9 residents utilized healthcare services more frequently than residents in code 2. However, when HCU patterns in codes 2 and 9 were investigated by race, no difference in HCU patterns was identified between Non-Hispanic White and Black residents in code 9, whereas Black residents in code 2 utilized healthcare services more frequently than Non-Hispanic White residents (a finding that approached significance, $\mathrm{p}<0.02$ ). This finding is consistent with that of Thorson and Powell (2000) who found that the African American OAs in their urban sample saw their primary care doctor with greater frequency in the past year compared to the Non-Hispanic White urban OAs. There was also no difference in overall medical burden between Black and Non-Hispanic White residents in code 9, but Black residents were sicker than Non-Hispanic White residents in code 2, which may explain the greater rates of healthcare utilization in urban Black OAs (i.e. a greater need). This finding may lend support to the literature indicating the presence of significant racial disparities in health in urban locations (Williams and Mohammed, 2009). Moreover, Black urban (code 2) OAs were significantly more likely to utilize public clinics compared to Non-Hispanic White urban OAs, and Black urban OAs were significantly less satisfied with their healthcare services compared to the Non-Hispanic White urban residents, findings not present in rural code 9. Such findings are consistent with the 
general rural/urban differences presented above, and continue to support the idea that type of healthcare services utilized and satisfaction with healthcare services may be critical variables to investigate as potential racial and residential HDs in the future.

Similar results to racial differences by county code were found when investigating the role of annual income on HCU patterns. Looking at the study sample as a whole, participants in lower annual income groups utilized healthcare services more frequently than individual in greater annual income groups, which is consistent to the results of Thorson \& Powell's (2000). Again, however, when investigating the differences in pattern of HCU for levels of annual income by individual county codes, HCU patterns did not differ between income groups in code 9 , whereas greater HCU was significantly associated with lower annual income groups in code 2. Further, the low income group in code 2 had greater overall medical burden (more chronic health conditions) compared to the higher income groups, whereas there was no difference in overall medical burden in code 9. In addition, although poorer urban OAs utilized healthcare services more frequently than urban OAs with higher annual incomes, they utilized public clinics more frequently and were less satisfied with their care.

Although the initial prediction that rural OAs would utilize healthcare services less frequently compared to urban older adults was not supported, the results suggest that one's race, income, and type of usual outpatient healthcare provider may all affect rates of HCU by level of rurality (county code). It is not sufficient to say urban OAs utilize healthcare services less often than rural OAs, when in fact, rural OAs with few chronic medical conditions may utilize healthcare services less than urban OAs in general; and within the urban OA sample, Black residents and residents in lower annual income 
groups utilize outpatient healthcare services more often than Non-Hispanic White urban residents and resident in greater annual income groups. Clearly race, annual income, and place of usual outpatient healthcare services are critical variables to better understanding differences in HCU patterns across rural and urban OAs. Moreover, there are a number of ways to compare rural and urban health, and the current results suggest that (1) what healthcare service options are available and (2) one's satisfaction with the healthcare services received, should be investigated in future research examining the effects of these two variables on physical and emotional health outcomes.

Given the similar patterns of race and income on $\mathrm{HCU}$, it is important to clarify the relationship between race and income in the current sample. Past literature has suggested that race may simply represent a proxy for income, given that Black individuals are overrepresented in low SES groups (Dressler, Oths, \& Gravlee, 2005). However, although the race and income variables in the current sample follow similar patterns in terms of $\mathrm{HCU}$, they clearly represent distinct variables equally important to the investigation of HDs in rural versus urban OAs. This is supported by the current findings that Black OA participants had significantly less income than Non-Hispanic White participants in both county codes 2 and 9 ; however, the racial and income differences observed in HCU patterns occurred only in county code 2, the most urban county. This finding supports the more recent consensus in HD literature stressing the need to investigate race and income separately (Dressler, Oths, \& Gravlee, 2005). Doing so "leaves open the possibility that race independently influences physical and emotional health through pathways such as personal experiences of discrimination or cultural differences in lifestyles (Kawachi, Daniels, \& Robinson, 2005, p. 347)." 
Researchers investigating differences in $\mathrm{HCU}$ patterns and access to healthcare across groups have differentiated between equitable and inequitable access to care (Arcury, et al., 2005). Equitable access includes demographic variables (e.g. age) and need variables (e.g. the number of chronic medical conditions) that account for variation in HCU. These variables are "equitable," because one would expect OAs and sicker individuals to utilize healthcare services more frequently. Inequitable access occurs when variables such as ethnicity (a social structure variable), health beliefs, and enabling resources (e.g. income), determine who receives medical care and the quality of medical care received. Although need factors, such as the number of chronic medical conditions were found to influence HCU patterns in the current study, the results clearly indicate that differences in HCU patterns in the current North Carolina sample are partially determined by inequitable factors, such as race and income, particularly in the most urban county. These results are unsettling and clearly stress the need for continued research focusing on developing public policy initiatives and interventions aimed at reducing such inequities.

\section{Culture:}

Another HD variable investigated in the current study was potential cultural beliefs held by rural OAs that may negatively affect health status and health outcomes in rural versus urban OAs. Previous literature often states rural residence, particularly rural OAs, have a unique culture focusing on hardiness and self-care that affects their health beliefs and help-seeking behaviors (Klugman, 2008). However, this belief is often mentioned but not measured. The current study investigated two measures of cultural 
beliefs hypothesized to be greater in rural compared to urban OAs, which may affect one's health beliefs and behaviors: (1) more negative attitudes towards doctors (NAs) and (2) greater self-efficacy. As predicted, level of rurality was significantly related to NAs, such that participants reported more NAs as rurality increased. This result is consistent with previous studies investigating the health beliefs of rural and urban OAs. In their study of OAs in Nebraska, Thorson and Powell (2000) found that the rural residents reported more medical skepticism than urban residents, and demonstrated less positive attitudes towards healthcare in general and in the efficacy of medical professionals.

Contrary to the initial prediction, however, urban OAs had higher self-efficacy scores on average compared to rural OA participants, a finding which approached significance $(p=0.017)$. This finding is inconsistent with the findings of Thorson and Powell (2000), who found that rural OAs reported greater independence in healthcare and less reliance on medical help compared to urban OAs. According to these researchers, the rural residents in this study were more likely to "tough it out." One possible reason for the opposite finding in the current study is the self-efficacy measure utilized. The Pearlin Mastery Scale is a general measure of self-efficacy, and does not pertain specifically to matters of health. The items asked by Thorson and Powell were specific to healthcare efficacy beliefs, both in terms of self-care and the efficacy of formal healthcare services.

However, although it is possible the current self-efficacy measure does not tap into health-related self-efficacy specifically, the finding that urban OAs in code 2 reported significantly higher levels of self-efficacy than the rural OAs in code 9 warrants further study. This is particularly true in light of the finding that greater self-efficacy is 
significantly associated with less HCU (discussed in more detail below). Understanding the relationship between general self-efficacy beliefs and HCU may help develop interventions aimed at increasing preventative health behaviors (e.g. exercising and healthy nutrition) and effective health-maintenance behaviors (e.g. effective selfmanagement of chronic illnesses, such as diabetes) in individuals who tend to over-utilize healthcare services. Clearly, future research is needed to investigate the role of selfefficacy on physical health status, health behaviors, and HCU; as well as investigate what differences between rural and urban OAs may be contributing to the greater self-efficacy beliefs found in the current urban OA sample.

In addition, future studies examining rural/urban differences should investigate a more valid measure of self-efficacy beliefs specific to health care. One possibility is to utilize measures of health locus of control. In general, the concept of locus of control, which is derived from social learning theory, relates to the expectations of who (or what) is responsible for outcomes. Having greater internal locus of control implies that a person believes what happens to him (positive or negative) is the consequence of his own actions, and thus is under his control (Wallhagen, Strawbridge, Kaplan, \& Cohen, 1994). In terms of health locus of control specifically, the belief is that those with greater internal health locus of control will be more actively involved in their own healthcare (Wallhagen, et al., 1994). Howat, Veitch \& Cairns (2006) found that the rural residents in their Australian sample reported greater levels of internal health locus of control (measured by the Multi-Dimensional Health Locus of Control Measure, MDHLC) compared to their urban sample. These researchers did not investigate the association between health locus of control and health status or health behaviors in this study. 
In addition to investigating differences in health beliefs by level of rurality, researchers have "called for studies of health values, beliefs, and attitudes of the rural elderly in order to clarify and explain differences in urban and rural older people's health care utilization (Thorson \& Powell, 2000, p. 49)." The current study predicted that selfefficacy and NAs would significantly relate to $\mathrm{HCU}$, such that greater self-efficacy and more NAs would lead to less HCU. Support was found for a significant relationship between self-efficacy and $\mathrm{HCU}$, in the predicted direction. That is, individuals with greater self-efficacy utilized outpatient services less frequently in the past year. Future studies need to further investigate the mechanisms behind this relationship. Is it that individuals with greater self-efficacy choose to take care of their health concerns on their own, versus seeking formal medical care, leading to less HCU; or is it that individuals with greater self-efficacy engage in healthier lifestyles overall (e.g. engage in preventative health behaviors, effective health maintenance behaviors, etc.), which decreases overall medical burden and hence the need for formal healthcare services, leading to less HCU? In the current sample, overall medical burden did not significantly mediate the relationship between self-efficacy and HCU; however, participants with greater self-efficacy were significantly healthier (had fewer chronic health conditions) compared to participants with less self-efficacy, which may lend more support to the latter hypothesis. Future research is needed to clarify this finding.

Negative attitudes towards medical doctors (NAs) on the other hand were not significantly related to $\mathrm{HCU}$ in the current sample. Although one would expect having more NAs would predict less HCU, this result is consistent with previous research investigating similar health belief variables. Thorson and Powell (2000) found that 
although their rural OA sample reported more medical skepticism than urban residents, and demonstrated less positive attitudes towards healthcare and in the efficacy of medical professionals, both groups reported receiving the services they need, and no difference was found in the frequency of outpatient primary care visits between the two groups.

Taken together, the results suggest differences exist in cultural values held by rural and urban OAs, and that such cultural beliefs can affect $\mathrm{HCU}$ and possibly other health behaviors. In turn, such belief may indirectly affect physical health status. Future studies are needed to develop more valid measures of health-related cultural beliefs in order to adequately investigate differences in the beliefs held between rural and urban OAs and how these health beliefs may negatively impact physical health status and patterns of HCU.

Study Question 2: Do rural elders have greater depression and do HD's account for this?

There were no differences in depression severity or rates of prevalent depression across level of rurality in the current sample. Likewise, there were no significant differences in rates of incident depression across the county codes at all three follow up waves. This finding is contrary to previous studies finding greater rates of depression in rural compared to urban residents (Murrell, Himmelfarb, \& Wright (1983); Probst, Laditka, Charity, et al., 2006; Schwab, Warheit, \& Holzer, 1974). In addition, although HDs in general significantly predicted depression, none mediated or moderated the relationship between rurality and depression. Follow-up analyses were conducted to help explain this non-significant finding and are discussed in detail below. In particular, it 
appears that race may be an important source of variability that could help clarify the lack of support for the initial hypothesis.

Given that income, education, and race were all significantly associated with level of rurality and depression in the current study, follow-up analyses were conducted to investigate whether or not these variables significantly interact with level of rurality on prevalent depression. Support was not found for the role of income or education as moderator variables. Race (Black vs. Non-Hispanic White), however, did significantly interact with rurality and depression, although at a more lenient significance value of $p<$ 0.05. When looking at the results for the Non-Hispanic White participants separately, the findings follow the pattern originally predicted, i.e. rates of prevalent depression increased as rurality increased. However, the opposite pattern was found for the Black participants, in which rates of prevalent depression decreased as rurality increased. Overall, this finding suggests that rural/urban residence in of itself does not capture the heterogeneity within residential groups and therefore cannot, on its own, adequately explain the relationship between where one lives and depression. Rather, other individual characteristics need to be investigated in conjunction with rural/urban county code. Ethnicity, in particular, may be a critical variable that can help clarify the role of rural/urban status on rates of prevalent depression. Further, the finding that depression increases as one's county code becomes more urban for Black participants, provides additional evidence that Black urban OAs are at a particularly high risk for both physical and emotional HDs. Interestingly, as discussed earlier, significant racial and income HDs were found only in code 2, in which urban Black residents reported a greater number of chronic health conditions and greater HCU compared to the urban Non-Hispanic White 
participants. It is possible that HDs may lead to greater rates of prevalent depression in urban Black and poorer urban OAs, although future studies are needed to investigate this hypothesis.

Another way to look at the current study results, however, is the relative lack of racial disparities (i.e., lack of significant differences between Non-Hispanic White and Black participants) in rural county code 9 . There were no differences between overall medical burden and HCU patterns between the rural Black and Non-Hispanic White participants, and in fact, rural Black OAs had lower rates of prevalent depression compared to the Non-Hispanic White residents. These findings raise the questions: what is different about the rural environment that may prevent racial HDs, and what are the characteristics of rural Black OAs, if any, that may buffer the negative physical and emotional health outcomes caused by HDs? Examining such questions may help researchers, clinicians, and public policy makers identify ways to prevent and buffer the negative effects of racial HDs in urban locations. There are several possibilities, which include differences in environmental factors between rural and urban settings, and differences in individual characteristics between rural and urban Black OAs, which will be discussed in more detail below.

For example, in their sample of rural Black adults in Tennessee, Linn and colleagues (1989) found that dissatisfaction with the community was more significantly associated with depression than stressful life events, whereas stressful life events were more significantly associated with depression in their urban Black sample compared to satisfaction with the community. The results of this study lead to a number of interesting questions. For example, are rural Black OAs faced with fewer stressful life events 
compared to urban Black elders due to the qualities of living in a rural environment? Or, do rural Black OAs have more effective coping skills or more social support to deal with stressful life events? Although the opposite pattern in rates of prevalent depression occurred for the Non-Hispanic White OA participants, similar variables (i.e. differences in the occurrence of stressful life events and satisfaction with the community) may also help explain the difference in rates of prevalent depression across rural/urban status in this group. Investigating both environmental and individual differences may help to explain the difference in rates of prevalent depression in rural versus urban Black and Non-Hispanic White OAs.

Many models have been developed in the literature to explain racial HDs, such as those seen in the current urban Black sample (both in terms of physical health variables and depression), which may help guide future research interested in understanding the role of race in rural versus urban depression. These models include: 1) a racial-genetic model $^{1}, 2$ ) a heath-behavior model $^{2} ; 3$ ) a socioeconomic model $\left.{ }^{3} ; 4\right)$ a psychosocial stress model; and 5) a structural-constructivist model (Dressler, Oths, \& Gravlee, 2005).

\footnotetext{
${ }^{1}$ The racial-genetic model suggests genetic differences in disease risk by race. It has had little support and has been found to have little explanatory power. This is $\backslash$ not surprising given that "race" is not a biological construct, and therefore it is difficult to find racial-genetic attributions of disease risk (Dressler, Oths, \& Gravlee, 2005).

${ }^{2}$ The health-behavior model suggests HDs in a population group arise as a result of discrete negative health behaviors adopted by more individuals in hat group compared to others, e.g. smoking, excessive alcohol intake, high caloric intake, and low physical activity - activities which often lead to obesity and other health conditions (Dressler, Oths, \& Gravlee, 2005). Studies have attempted to investigate the combined effect of negative heath behaviors in reducing racial disparities, with results being mixed. Taken together, however, the results of such studies suggest negative health behaviors (alone or combined) cannot fully explain racial HDs (Dressler, Oths, \& Gravlee, 2005).

${ }^{3}$ The SES model suggests racial HDs are confounded with SES, as Blacks are overrepresented among lower SES groups. However, numerous studies have found that controlling for SES fails to completely account for racial disparities, despite a reduction in the magnitude of group differences (Dressler, Oths, \& Gravlee, 2005).
} 
Recent studies have found increased support for the psychosocial stress and structuralconstructivist models, which may help to explain the current findings (Dressler, Oths, \& Gravlee, 2005).

The psychosocial-stress model suggests stress associated with racism may lead to racial HDs. Here, racism is equated to the more general term of "ethnic discrimination," and defined as the "unfair treatment received because of one's ethnicity, where 'ethnicity' refers to various groupings of individuals based on race or culture of origin (Brondolo, Gallo, \& Meyers, 2009, p. 3)." There are two primary forms of racism that may lead to stress in the psychosocial-stress model, which are institutional racism, such as less access to resources, (including high paying jobs and living in neighborhoods that are safe to walk for exercise or that have fresh produce markets); and perceived racism, which is the self-report of individuals regarding their personal experiences of discriminatory acts (Dressler, Oths, \& Gravlee, 2005).

Future research investigating differences in both institutional and perceived racism experienced by rural versus urban Black OAs may shed light onto the greater prevalence of HDs and rates of prevalent depression found in the urban versus rural Black participants in the current study. Researchers have recently stressed the need to investigate variations within groups to understand how factors such as racism disproportionately relate to depression across members of the same group (Brondolo, Gallo, \& Myers, 2009). Investigating environmental differences by rural/urban status is one way to investigate variations in HDs and depression within the Black OA population (Klonoff, 2009, Do, et al., 2008; Redwood, et al., 2010). Overall, the results of the current study suggest ethnicity is a critical variable that needs to be included in future 
studies examining the relationship between level of rurality and HDs, and level of rurality and depression.

In addition to the environmental differences in rural/urban locations that may be associated with depression in Black OAs, future research also needs to investigate differences in the individual characteristics (e.g. coping mechanisms and psychosocial supports) between rural and urban Black elders that may buffer against the effects of life stressors, racial HDs, and racism. Brondolo, ver Halen, Pencille, Beatty, \& Contrada (2009) recently stressed the need to investigate differences in coping and social support within and across ethnic groups as a means to develop effective interventions to help buffer against the stress caused by racism, which negatively impacts physical and emotional health.

In the current study, support was not found for a significant interaction between perceived social support, level of rurality, and race; however, it is interesting to note that the highest percentage of Black residents with low perceived social support occurred in the most urban group (code 2), compared to the three other codes, whereas the highest percentage of Non-Hispanic White residents with low perceived social support occurred in the most rural group (code 9), a finding which parallels the rates of prevalent depression by race and level of rurality. Unfortunately, as will be described in more detail below, the perceived social support measure used in the current study may lack explanatory power. It may be that social support differs between urban and rural Black and Non-Hispanic White OAs, and that this difference significantly impacts the development of depression in these groups. Future research is needed to investigate this hypothesis, as well as investigate multiple types of social support (e.g. instrumental, 
informational, social influence, social networks, etc.), as they may impact the associations between race, rurality, and depression differently.

Research focusing on the structural-constructivist model may also provide insight into the racial differences found in rates of prevalent depression between rural and urban OAs in the current sample. This model suggests that how individuals of particular "racial" groups define having a "good life," and the degree to which those individuals are able in their own behaviors to approximate that lifestyle (i.e. a "cultural consonance in lifestyle"), may explain racial disparities in depression (Dressler, Oths, \& Gravlee, 2005, p. 242). In this sense, racial depression disparities occur when the life goals of persons in "communities of color" are limited by racial discrimination (Dressler, Oths, \& Gravlee, 2005). It is possible the gap between a participant's personal description of a good life and the approximation of such a life is greater in urban compared to rural Black OAs, although future research is needed to investigate this hypothesis and the factors that would lead to such a gap in the urban sample.

Moreover, given that ethnicity and race are social constructs that are primarily culturally and historically defined (O'Neil, 2006), the social-constructivist model leads to an additional question. Is there less of a difference in Black and Non-Hispanic White social identity in rural locations, and if so, is this due to social identity being more heavily influenced by factors associated with living in a rural environment (e.g. rural culture, shared residential disparities such as limited healthcare options, etc.)? The answer to this question may help to explain the pattern whereby Blacks and NonHispanic White participants did not differ on key study variables (HCU, overall medical 
burden, satisfaction with healthcare) in county code 9 , but did significantly differ in county code 2 . Future studies are needed to investigate this question.

Overall, these results suggest that although rural/urban status (measured by the ERS rural/urban continuum codes), in and of itself, is not associated with depression severity or rates of prevalent depression, differences in rates of prevalent depression across level of rurality are present when one considers other critical participant characteristics such as race. Future studies investigating differences in depression and health factors in rural and urban OAs will need to continue to include the examination of such variables.

Finally, in an attempt to better understand of the effect of overall medical burden and HCU on the association between level of rurality and depression, the potential moderating effects of physical disability and perceived social support on this relationship were investigated. These two variables were not found to impact the association between level of rurality and HDs on depression in the current sample. In terms of physical disability, no previous studies were identified that investigated potential moderating effects of physical disability on the association between HD variables and depression. However, Yochim, Kerkar, and Lichtenberg (2006) investigated the potential mediating effect of physical limitations on the association between CVR and depression in a sample of urban African American OAs, and did not find support for the role of physical disability as a mediator of this relationship.

In terms of perceived social support, the current findings are contrary to those of Fukukawa and colleagues (2004) who found that perceived emotional support from family members buffered the relationship between health problems (having a "major 
injury or disease") and depression. The non-significant finding in the current study may have resulted from the perceived social support assessment measure utilized, in that it may not have been sensitive enough to adequately measure differences in the experience of social support between rural and urban OAs. The current measure was comprised of only two individual items, and the Cronbach's alpha was only .55. Future studies need to investigate the potential moderating effects of perceived social support on the association between HDs and depression, and the association between HDs and level of rurality on depression utilizing a more extensive and valid social support measure.

Further, the current measure of perceived social support was not able to capture the full range of pathways through which social support can impact health (e.g. through access to health information, social influence (positive and negative), and instrumental support for accessing healthcare). It is possible the current study underestimated the role of social support to some extent on the association between HDs, rurality and depression. There is growing consensus that social support is a multidimensional concept (Fukukawa, et al., 2004). Therefore, the type of support (e.g. perceived, instrumental, informational, social influence, social networks, activity participation, etc.) needs to be considered when examining the relationships between social support, physical health, and depression.

Study question 3: What is the value of a more specific rural/urban definition?

A novel component of the current study was the use of a more varied and specific measure of rural/urban status. The current study utilized the ERS rural/urban continuum codes, which consists of 9 codes based on county population size and the proximity of the county to a metropolitan center (see Appendix D). Code 1 equates to the most urban of 
counties and code 9 equates to the most rural of counties. Such a tiered coding system is particularly important when investigating the effects of health status and HDs by place of residence, given the discrepancies in healthcare often found the further one resides from a metropolitan location (Washington State Department of Health, 2009). The 9 ERS codes can be broken down into three conceptual categories: 1-3 are more urban, 4-7 equate to more suburban locations, and 8-9 are more rural.

The current study included a highly urban code (code 2), two suburban codes (code 6 and 7), and one rural code, (code 9), which is the most rural code. Although the overarching hypothesis investigating differences in HDs and depression across these four rural/urban continuum codes was not supported, there were a number of important differences across the codes, supporting the value of a tiered rural/urban coding system and suggesting that the ERS rural/urban continuum codes should continue to be utilized in future studies.

To begin, the difference found between codes 2 and 9 on the HCU variable may suggest that for some health variables, disparities may only occur between the most urban and most rural codes. This is an important distinction, because if futures studies dichotomize rural/urban status more broadly (e.g. individuals that live in metropolitan statistical areas versus those that do not), which was often the case in past studies, they may lack the sensitivity and variability in their definition of rural and urban needed to identify important disparities between residential groups.

In addition, researchers have more recently suggested that for some health variables both highly urban and highly rural residency are associated with HDs when compared to living in more suburban counties (Ebernhardt \& Pamuk, 2004). Such 
differences cannot be investigated without the use of a more sensitive and varied measure of rural/urban that can differentiate between levels of rural, suburban, and urban status. In the current study, although no significant difference was found between the four urban codes on the overall medical burden variable fewer residents in code $6(12.8 \%)$ and 7 (12.2\%) reported three or more chronic health conditions (the most severe level of medical burden in the current variable) compared to codes $2(13.7 \%)$ and $9(16.1 \%)$. This result is consistent with prevalence rates reported by Ebernhardt and Pamuk (2004), who found that $18 \%$ of rural adults reported having chronic conditions that caused activity limitation compared with only $13 \%$ of suburban adults (Ebernhardt \& Pamuk, 2004).

Place of usual healthcare also differed across the four county codes such that code $6(80 \%)$ and $7(84 \%)$ residents were more likely to utilize private practice offices compared to residents in codes $2(63 \%)$ and $9(52 \%)$, suggesting possible discrepancies in the type of healthcare services available between rural, urban, and suburban residents. This difference may lend additional support to the hypothesis that highly rural and highly urban residents are at the greatest HD risk. Such discrepancies in one's usual place of healthcare is a particularly important finding given that participants using private practice offices tend to be healthier (report fewer chronic health conditions), use health services less often, and are more satisfied with their healthcare compared to participants who utilize public clinics for their usual place of healthcare.

The findings above supports the value of having a more varied and sensitive measure of rurality, as the more rural residents in code 9 (i.e. small county population and further from a metropolitan center) are quite different from county code 7 , only two 
codes higher. If researchers examined samples consisting of only code 2 and 7 residents they might not see the types of differences observed between rural, suburban, and urban residents in the current study. Previous studies likely would have considered code 7 "sufficiently" rural for a comparison group to urban residents, as it is a county code outside an MSA. Further, as stated previously, past analyses using the Duke EPESE data considered codes 6, 7, and 9 "rural," combining these three codes in analyses comparing rural and urban OAs on a number of health variables (Blaser, et al., 1995).

Finally, there were clear trends across the county codes in the cultural variables investigated, such that self-efficacy increases as rurality decreases and NAs increase as rurality increases.

Taken together, it appears that rural status may not always confer a HD. More rural residents may be at a health disadvantage on some health variables compared to urban residence, whereas urban residents may be at a health disadvantage compared to rural residence on other health variables; and even further, both the most rural and most urban residents may be at a health disadvantage compared to residents living in suburban counties on other health variables. The only way to determine which HD variables need to be addressed where, is to utilize a more sensitive and varied measure of rural/urban status. These complex findings are consistent with those summarized by Ebernhardt and Pamuk (2004), who found that differences by rural/urban residence existed for some health measures but not others, and found there was not always a clear rural-to-urban gradient in the presence of HDs.

Perhaps most importantly, the results of the current study suggest that it may not be rural/urban status alone that is associated with HD variables, but rather rural/urban 
status in conjunction with other demographic and environmental variables, such as race, income, and the health services available. Again, however, many of the differences identified were between the most rural and urban county code, suggesting the need for a more detailed definition of level of rurality. Although, some clear trends were identified across the four county codes, such as the differences in rates of prevalent depression by race (Non-Hispanic White vs. Black). In summary, the use of the ERS rural/urban continuum codes was a strength of the current study, which allowed for the investigation of HDs and depression by level of rurality utilizing a more sensitive and detailed measure of rural/urban status. It will be important for future research investigating differences in rurality to continue to use such a detailed and varied measure of rural/urban status.

\section{Limitations and future directions}

This was the first study to directly assess differences in depression severity and the possible mediating and moderating role of $\mathrm{HD}$ variables on the relationship between level of rurality and depression utilizing a more sensitive measure of rural/urban status. In the current sample, rural/urban residence, in and of itself, was not significantly associated with depression severity, and HDs were not found to mediate or moderate the relationship between level of rurality and depression. In addition, other than $\mathrm{HCU}$, the HD variables investigated did not differ solely by rural/urban status. One conclusion of this study, however, appears to be that rural/urban status, in and of itself, does not directly affect HDs and depression due to the heterogeneity of individuals within rural and urban counties. Rather, the relationship is more complex and other variables (e.g. race) need to be investigated in conjunction with place of residence to understand how HDs and depression differ across rural and urban groups. 
There are a number of limitations in the current study that must be discussed as well as several important future directions that researchers interested in the relationship between level of rurality, HDs, and depression should consider. To begin, this study focused on only one region of the United States (north central North Carolina). Although limiting the sample to a specific U.S. region and contiguous counties allows for a more targeted rural vs. urban comparison, it does not allow for comparisons across different regions in the United States. Rural and Urban OAs are very heterogeneous, and therefore, this sample may have unique characteristics that make it less generalizable to the rural OAs in other regions of the United States. For example, the north central North Carolina population utilized in this study is primarily populated by Non-Hispanic White and Black OAs, with over $50 \%$ of the population being Black. The current findings may not generalize to U.S. regions more heavily populated with Non-Hispanic White Americans or other minority groups, such as Hispanic-Americans. This is particularly relevant given the significant role of race in the current study. Likewise, differences in the region characteristics itself (e.g. differences in access to healthcare, amount of racial discrimination, and the general cultural norms and beliefs, etc.) may lead to more significant differences in the health variables between rural and urban OAs in other regions of the country.

Further, although the extensive sampling procedures utilized in the current study resulted in a representative sample of north central North Carolina community dwelling OAs, the current sample only consisted of individuals healthy enough to be living in the community. It is possible that the association between health variables and depression across rural/urban status may be more significant in a more varied sample in terms of health status, i.e. with the inclusion of OAs from more medically compromised settings such as assisted living and nursing home facilities, or OAs recruited from primary care 
offices and medical rehabilitation centers. Future research should investigate the HDdepression association across a more diverse rural/urban sample by including OAs from the above listed settings in addition to community-dwelling elders.

Although no direct differences were found by level of rurality in terms of depression and most of the HD variables investigated, this was the first study to utilize a more sensitive and varied definition of rural/urban status. This represents one strength of the current study given that a significant limitation in the existing literature on rural/urban differences is the lack of a standardized and sensitive measurement of place of residence. However, the current sample consisted of only four out of nine ERS county codes at Wave 1, and three out of nine codes at Wave 2. Although these codes spanned urban, suburban, and rural counties, suggesting sufficient variability in level of rurality for the purposes of the current study, future studies including a greater number of the 9 tiers would allow for more variability in rural/urban status and may yield additional differences and important findings.

The current study was also unable to control for length of rural/urban residency, and the current assessments of rural/urban status only applied to a participant's residency at the time of assessment. Such classifications do not allow for the possibility that an individual who lived the majority of his or her life in a rural environment, exposed to the many possible HDs associated with rural communities, including rural cultural beliefs, recently moved to an urban area, or vice versa. Schwab, Warheit, and Holzer (1974) label this confound as "selective migration." These authors empirically showed the importance of distinguishing between current residence and place of origin. Their rural 
sample originally from a rural area had the highest rates of depression, whereas both their urban and rural samples originally from an urban area had the lowest rates of depression.

Assessing selective migration patterns may be particularly relevant when investigating HDs, particularly if there are differences in rural and urban health culture. Averill (2005) found that length of rural residency affects one's perceptions and definition of health. Rural residents of over 40 years "viewed health as avoidance of contact with the health care system and ability to be active and autonomous in daily life, regardless of chronic health conditions. More recent retirees to the areas tended to define health as a function of proper diet, exercise, and regular check-ups with their providers (p. 15)." Therefore, future research should also assess the length of current rural/urban residency, as well as where the OA participants grew up (in a rural or urban county) to determine if such variables better predict differences in HDs and rates of depression across current "rural" and "urban" residency.

The current study could also have been improved by the inclusion of wellvalidated measures of perceived social support and NHBs, as well as a self-efficacy measure that more directly assessed health related self-efficacy (e.g. a measure of health locus of control). The two-item perceived social support measure utilized in this study may not have been sensitive enough to fully investigate the role of perceived social support on the relationships between level of rurality, HDs, and depression. Future research using a more comprehensive and previously validated perceived social support measure, along with measurements of other types of social support (e.g. instrumental, social influence, social networks, etc.) may help better explain the effects of social support on such rurality/HD/race/depression relationships. 
The NHB measure utilized was created for the purpose of this study and was not a validated measure. Further, the measure only included smoke risk, alcohol risk, and exercise risk behaviors, which may not have fully captured the range of NHB differences across level of rurality. Perhaps other health behaviors, such as a eating a healthy diet, proper medication use and disease management are more highly associated with depression severity. In addition, the included items were all based on self report, and therefore could have led to underreporting of risk behaviors, particularly for socially sensitive behaviors such as heavy drinking. A more valid and comprehensive measure of negative health behavior risk, possibly including an informant report should be utilized in future studies.

Likewise, the Pearlin Mastery Scale was utilized to assess self-efficacy beliefs in the current study. However, there may be more appropriate and well-validated measures to assess self-efficacy beliefs regarding health behaviors, health status, and the efficacy of formal medical care, such as the Multi-dimensional Health Locus of Control Scale (Howat, Veitch \& Cairns, 2006). Further, the only other assessment of "culture" in the current study was a 10-item questionnaire assessing one's negative attitudes towards doctors. Future studies investigating differences in rural and urban culture that may affect health behaviors and that may be associated with a greater risk for developing depression are needed.

Another limitation of the current study was that access to care was not directly analyzed. There is an extensive literature suggesting rural OAs are faced with significantly more barriers to accessing adequate healthcare compared to their urban counterparts. It is possible that more objective measures of access to care, as well as 
one's perceived beliefs regarding access to care, would have differed by county code in the current sample and may have been related to depression severity. A more direct assessment of access to care would also have allowed for a deeper understanding of other study results, such as the greater HCU in county code 9 versus 2 . For example, it is assumed that the current rural OAs did not experience difficulties accessing healthcare services to the degree often reported in the literature on rural HDs. Objective items querying about access to care would have helped clarify this picture. Future studies investigating the role of HDs on the relationship between level of rurality and depression should include more objective measures of access to care (e.g. number of hospitals, medical specialists, and general physicians in the area by type), in addition to one's perceived barriers to/difficulty accessing that care.

Further, the current study only investigated differences in depression, which provided only one index of mental health. It is possible that being exposed to HDs relates more to other measures of psychological well-being, such as life satisfaction, overall quality of life, and self-efficacy. Future researchers investigating differences in rural and urban mental health should investigate these constructs in addition to depression severity.

All of the questions asked in the current study were based on self-report. Due to the reliance upon respondents' self-report of depressive symptoms and health conditions, it is possible some symptoms were underreported. Further, there is a literature that examines the stigma surrounding mental illness among OAs, rural residents, and Black individuals (Hudson, 2009; Lyness, Cox, Curry, Conwell, King, \& Caine, 1995; Simmons, HuddlesonCasas, \& Berry; Voaklander, Thommasen, \& Michalos, 2006). Given over half of the current sample was Black and a significant portion was very rural, it is possible that social stigma 
may have impacted the self-report of depressive symptomotology and other variables such as physical disability.

Possibly the most important finding of the current study is that to understand differences in health and depression between rural and urban OAs, future researchers will need to include other critical variables accounting for the heterogeneity of individuals within each group. One related finding was the association found between race, county code, and depression. The fact that these analyses were exploratory in nature emphasizes the need for replication. Further, the inability of the current study to fully explain this association reinforces the need for future studies to investigate the factors related to differences in racial disparities (physical and emotional) by level of rurality. Given race is socially constructed, it will be imperative that researchers in the future clearly specify what they mean by race, and how race was defined and measured.

According to Dressler, Oaths, \& Gravlee (2005), rigorous tests of the precise causal mechanisms involved in racial disparities are the exception, not the rule. Researchers have recently stressed the need to investigate variations within groups (e.g. rural/urban status of Black elders) to understand how factors such as racism may disproportionately affect members of the groups and account for differences in health and depression within the group (Brondolo, Gallo, \& Myers, 2009). However, according to Brondolo and colleagues (2009), although there is a "clear rationale for hypothesizing that racism and ethnic discrimination affect health, empirical demonstrations of these effects are still in their early stages. Further work is needed to understand the mechanisms through which racism and ethnic discrimination relate to health (p. 2)."

The current results lead to a number of questions that can guide future research suggestions. Is there something about the urban environment (e.g. more institutional 
racism or more negative housing/environmental conditions) that leads to more depression in urban compared to rural Black OAs? Is there something about the individual characteristics of rural Black OAs (e.g. more social support or more effective coping mechanisms to deal with racism) that leads to less depression in rural Black OAs? Do rural Black residents approximate their desired life goals more closely compared to urban Black resident? Is there less of a difference in Black and Non-Hispanic White social identity in rural versus urban OAs; and is this due to social identity being more heavily influenced by shared rural culture and history versus the culture and history attributed to one's race? Future studies should work to clarify the interaction between race and level of rurality on depression. Doing so will allow for more tailored and effective screening methods, prevention efforts, and treatment interventions for depression in diverse groups of OAs, and help to explain the mechanisms by which (socially constructed) race influences physical and emotional health across rural and urban status.

\section{Implications:}

This study contributes to the existing literature on HDs and depression, and points to the importance of including individual characteristics (e.g. race, income, place of usual healthcare, etc.) and environmental variables (e.g. types of healthcare services available, institutional racism, etc.) when investigating differences in health and depression between rural and urban OAs. Including variables that account for the heterogeneity of residents within rural/urban counties will be critical to understanding the differences across levels of rurality, and will help future researchers, clinicians, and public policy makers identify specific groups at particularly high risk for facing physical and emotional health 
problems. For example, the results of the current study suggest that Black OAs in urban counties may face a greater number of and severity of racial HDs, and are at a particularly high risk for depression.

The current results also have implications relevant for clinical interventions aimed at reducing HDs and depression in specific population groups, and interventions aimed at buffering the effects of HDs (residential, racial, SES) when present. Previous literature suggests increasing self-efficacy and social support may decrease the negative effects of HDs. For example, in terms of Black OAs, specifically, increasing Black OAs' ability to cope with both institutional and perceived racism may decrease the negative effects of racial HDs. Future research is needed to investigate possible intervention strategies as well as the efficacy of such interventions.

Eliminating HDs (physical and emotional) across groups, e.g. residential, racial and income groups was a major goal of "Healthy People 2010," (the US health agenda set by Congress), and will likely remain a main objective of Healthy People 2020 (Brondolo, Gallo, \& Meyers, 2009). However, despite increased research attention on HDs, investigators have yet to clearly identify and fully understand the factors that create and maintain disparities in health status (physical and emotional; Brondolo, Gallo, \& Meyers, 2009). Consequently, it is difficult for public policy makers and clinicians to develop effective interventions aimed at the pathways that lead to HDs or interventions aimed at offsetting the effects of HDs on health outcomes (Brondolo, Gallo, \& Meyers, 2009). Continuing to investigate the differences between rural and urban OAs, including individual characteristics (e.g. race, coping abilities, social support) and environmental 
characteristics (e.g. living conditions, healthy-living options, safety), may increase our understanding of the factors that lead to disparities in health and depression.

Further, specifically understanding the factors that lead to disparities in the rates of prevalent depression across OA groups needs further study. The goal of the current study was to investigate the differential role of HD variables on depression rates in rural versus urban OAs, in order to increase understanding of the variables that may account for differences in depression rates and severity across different OA populations. Although there were no differences in the severity and rates of prevalent depression across the current county codes, the results suggest there are important differences within the rural and urban groups that can help explain differences across these OA samples (e.g. rural vs. urban Black OAs, and Black vs. Non-Hispanic White urban OAs). Future research is needed to understand what factors influence such differences. Doing so will aid in the development of more tailored screening measures and more tailored prevention and treatment interventions targeted at identified at-risk groups. 


\section{REFERENCES}

Aderibigbe, Y. A., Bloch, R. M., \& Pandurangi, A. (2008). Emotional and somatic distress in Eastern North Carolina: Help-seeking behaviors. International Journal of Social Psychiatry, 49: 126-141.

Alcohol. (2008). Centers for Disease Control and Prevention: Department of Health and Human Services. Retrieved: August 1, 2009:

http://www.cdc.gov/alcohol/faqs.htm.

Alexopoulos, G. S., \& Latoussakis, V. (2004). Depression comorbidity with COPD. Psychiatric Annals, 34: 289-296.

Alexopoulos, G. S., Meyers, B. S., Young, R. C., Campbell, S., Silbersweig, D., \& Charlson, M. (1997). 'Vascular depression' hypothesis. Archives of GeneralPsychiatry, 54, 915-922.

Anderson, J. E., \& Prior, S. (2007). Nutrition and aging. Food and Nutrition Series: Health. Colorado State University. Extension: No. 9.322.

Arcury, T. A., Gesler, W. M., Preisser, J. S., Sherman, J., Spencer, J., \& Perin, J. (2005). The effects of geography and spatial behavior on health care utilization among the residents of a rural region. Health Service Research, 40: 135-155

Arthur, H. M. (2006). Depression, isolation, social support, and cardiovascular disease in older adults. Journal of Cardiovascular Nursing, 21: S2-S7. 
Averill, J. B. (2005). Studies of rural elderly individuals: Merging critical ethnography with community-based action research. Journal of Gerontological Nursing, December, 2005: 11-18.Baldwin, R., Jefferies, S., Jackson, A., Sutcliffe, C., Thacker, N., Scott, M., \& Burns, A. (2004). Treatment response in late-onset depression: Relationship to neropsychological, neuroradiological, and vascular risk factors. Psychological Medicine, 34: 125-136.

Baumeister, H. \& Harter, M. (2007). Mental disorders in patients with obesity in comparison with healthy probands. International Journal of Obesity, 31: 1151164.

Beekman, A. T. F., Copeland, J. R. M., \& Prince. M. J. (1999). Review of community prevalence of depression in later life. The British Journal of Psychiatry, 174: 307-311.

Beekman, A. T. F., Pennix, B. W. J. H., Deeg, D. J. H., Ormel. J., Braam, A. W., \& van Tilburg, W. (1997). Depression and physical health in later life: Results from the Longitudinal Aging Study Amsterdam (LASA). Journal of Affective Disorders, 46: $219-231$.

Black, S. A., Markides, K. S., \& Miller, T. Q. (1998). Correlates of depressive symptomatology among older community-dwelling Mexican Americans: The Hispanic EPESE. Journals of Gerontology: Series B: Psychological Sciences and Social Sciences, 53B: S198-S208.

Blazer, D. G. (2003). Depression in late life: Review and commentary. The Journals of Gerontology, 58A: 249-265. 
Blazer, D., Burchett, B., Service, C., \& George, L. K. (1991). The association of age and depression among the elderly: An epidemiologic exploration. Journal of Gerontology: Medical Sciences, 46: M210-M215.

Blazer, D., George, L. K., Landerman, R., Pennybacker, M., Melville, M. L., Woodbury, M., Manton, K. G., Jordan, K., \& Locke, B. (1985). Psychiatric Disorders: A rural/urban comparison. Archives of General Psychiatry, 42: 651-656.

Blazer, D., Hughes, G., \& George, L. (1987). The epidemiology of depression in an elderly community population. The Gerontologist, 27: 281-287.

Blazer, D. G., Kessler, R. C., McGonagle, K. A., \& Swarts, M. S. (1994). The prevalence and distribution of Major Depression in a national community sample: The National Comorbidity Survey. American Journal of Psychiatry, 151: 979986.

Blazer, D. G., Landerman, L. R., Fillenbaum, G., \& Horner, R. (1995). Health services access and use among older adults in North Carolina: Urban vs rural residents. American Journal of Public Health, 85: 1384-1390.

Blazer, D. G., Moody-Ayers, S., Craft-Morgan, J., \& Burchett, B. (2002). Depression in diabetes and obesity: Racial/ethnic/gender issues in older adults. Journal of Psychosomatic Research, 53: 913-916.

Bosworth, H. B., Bartash, R. M., Olsen, M. K., \& Steffens, D. C. (2003). The association of psychosocial factors and depression with hypertension among older adults. International Journal of Geriatric Psychiatry, 18: 1142-1148.

Brondolo, E., Gallo, L. C., \& Myers, H. F. (2009). Race, racism, and health: Disparities, mechanisms, and interventions. Journal of Behavioral Medicine, 32: 1-8. 
Brondolo, E., Ver Halen, N. B., Pencille, M., Beatty, D., \& Contrada, R. H. (2009). Coping with racism: A selective review of the literature and a theoretical and methodological critique. Journal of Behavioral Medicine, 32: 64-88.

Buys, L., Roberto, K. A., Miller, E., \& Blieszner, R. (2008). Prevalence and predictors of depressive symptoms among rural older Australians and Americans. Australian Journal of Rural Health, 16: 33-39.

Cairney, J., Corna, L. M., Wade, T, \& Streiner, D. L. (2007). Does the frequency of contact with general physicians reduce feelings of mastery in older adults? Journal of Gerontology, PSYCHOLOGICAL SCIENCES, 62B: P226-P229.

Camacho, T. C., Roberts, R. E., Lazarus, N. B, Kaplan, G. A., \& Cohen, R. D. (1991). Physical activity and depression: Evidence from the Alameda County Study. American Journal of Epidemiology, 134: 220-231.

Carney, R. M., \& Freedland, K. E. (2008). Depression in patients with coronary heart disease. The American Journal of Medicine, 121, S20-S27.

Carruth, A. K., \& Logan, C. A. (2002). Depressive symptoms in farm women: Effects of health status and farming lifestyle characteristics, behaviors, and beliefs. Journal of Community Health, 27: 213-228.

Comstock, G. W., \& Helsing, K. S. (1976). Symptoms of depression in two communities. Psychological Medicine, 6: 551-563.

Cornoni-Huntley, J., Blazer, D., Lafferty, M., Everett, D., Brock, D., Farmer, M. (1990). Established Populations for the Epidemiologic Studies of the Elderly: Resource Data Book. Volume II. Bethesda, MD: National Institute on Aging. 
Cornoni-Huntley, J., Blazer, D. G., Service, C., \& Farmer, M. E. (1990). Introduction, p. 8-19. Established Populations for the Epidemiologic Studies of the Elderly: Resource Data Book. Volume II. Bethesda, MD: National Institute on Aging. Covey, L. S., Glassman, A. H., \& Stetner, F. (1998). Cigarette smoking and Major Depression. Journal of Addictive Diseases, 17: 35-46.

Coward, R. T. \& Lee, G.R. (1984). An introduction to aging in rural environments. In R. T. Coward and G. R. Lee (Eds.), The Elderly in Rural Society (p. 3-23). New York: Springer Publishing.

Cromartie, J., \& Bucholtz, S. 2008. Defining the "rural" in rural America. Amber Waves: The Economics of Food, Farming, Natural Resources, and Rural America. http://www.ers.usda.gov/AmberWaves/June08/Features/RuralAmerica.htm. Retrieved: July, $27^{\text {th }} 2009$.

Crowell, B. A., George, L. K., Blazer, G. D., \& Landerman, R. (1986). Psychosocial risk factors and urban/rural differences in the prevalence of major depression. British Journal of Psychiatry, 149: 307-314.

DeSocio, J., Kitzman, H., \& Cole, R. (2003). Testing the relationship between self0agency and enactment of health behaviors. Research in Nursing and Health, 26: $20-29$.

Do, D. P., Finch, B. K., Basurto-Davilla, R., Bird, C., Escarce, J., \& Lurie, N. (2008). Does place explain racial health disparities? Quantifying the contribution of residential context to the Black/White health gap in the United States. Social Sciences and Medicine, 67: 1258-1268. 
Dong, C., Sanchez, L. E., \& Price, R. A. (2004). Relationship of obesity to depression: A family-based study. International Journal of Obesity, 28: 790-795.

Dressler, W. W., Oths, K. S., \& Gravlee, C. C. (2005). Race and ethnicity in public health research: Models to explain health disparities. Annual Review of Anthropology, 34: 231-242.

Eberhardt, M. S., Ingram, D. D., Makuc, D. M., Pamuk, E. R., Fried, V. M., Harper, S. B., Schoenbom, C. A., \& Xia, H. (2001). Urban and rural health chartbook. Health, United States, 2001. Hyattsville, Maryland: National Center for Health Statistics.

Ebernhardt, M. S., \& Pamuk, E. R. (2004). The importance of place of residence: Examining health in rural and nonrural areas. American Journal of Public Health, 94: $1682-1686$.

EPESE Composite measures and other commonly used measures: Description and documentation of original variables with missing values imputed. (1999). Study document provided by Duke EPESE investigators. Received on April 192010.

Fabre, I., Oppenheim, A., Gallarda, T., Meder, J. F., de Montigny, C., Olie, J. P., \& Poirier, M. F., (2004). Antidepressant efficacy and cognitive effects of repetitive transcranial magnetic stimulation in vascular depression: An open trial. International Journal of Geriatric Psychiatry, 19: 833-842.

Farmer, M. M., \& Ferraro, K. F. (2005). Are racial disparities in health conditional on socioeconomic status? Social Sciences and Medicine, 60: 1991-2004.

Farmer, M. E., Locke, B. Z., Moscicki, E. K., Dannenberg, A. L., Larson, D. B., \& Radloff, L. S., (1988). Physical activity and depressive symptoms: The 
NHANES I epidemiologic follow-up study. American Journal of Epidemiology, 128: $1340-1351$.

Ferreira, V. M., \& Sherman, A. M. (2006). Understanding associations of control beliefs, social relations, and well-being in older adults with osteroarthritis. International Journal of Human Development, 62: 255-274.

Fischer, L. R., Wei, F., Rolnick, S. J., Jackson, J. M., Rush, W. A., Garrard, J. M., Nitz, N. M., \& Luepke, L. J. (2002). Geriatric depression, antidepressant treatment, and healthcare in a health maintenance organization. Journal of American Geriatric Society, 50: 307-312.

Flegal, K. M. (1990). Agreement between two dietary methods in the measurement of alcohol consumption. Journal of Studies on Alcohol, 51: 408-414.

Foley, D. J., Fillenbaum, G. G., \& Service, C. (1990). Chapter 3: Physical Functioning, p. 34-50. Established Populations for the Epidemiologic Studies of the Elderly: Resource Data Book. Volume II. Bethesda, MD: National Institute on Aging.

Fried, L. P., McNamara, R. L., Burke, G. L., \& Siscovick, D. S. (1997). Heart health in older adults: Import of heart disease and opportunities for maintaining cardiac health. Western Journal of Medicine, 167: 240-246.

Fukukawa, Y., Nakashima, C., Tsuboi, S., Niino, N., Ando, F., Kosugi, S., \& Shimokata, H. (2004). The impact of health problems on depression and activities in middleaged an older adults: Age and social interactions as moderators. The Journals of Gerontology, 59B: P19-P26. 
Gaston, N. W., Mardis, A, Gerrior, S., Sahyoun, N., \& Anand, R. S. (1999). A focus on nutrition for the elderly: It's time to take a closer look. Nutrition Insights, Insight 14: USDA Center for Nutrition Policy and Promotion.

George, L. K., Blazer, D. G., Hughes, D. C., \& Fowler, N. (1989). Social support and the outcome of major depression. British Journal of Psychiatry, 154: 478-485.

Goldston, K., \& Baillie, A. J. (2008). Depression and coronary heart disease: A review of the epidemiological evidence, explanatory mechanisms and management approaches. Clinical Psychology Review, 28: 288-306.

Glassman, A. H., Helzer, J. E., Covey, L. S., Cottler, L. B., Stetner, F., Tipp, J. E., \& Johnson, J. (1990). Smoking, smoking cessation, and major depression. JAMA, 264: 1546-1549.

Gorman, B. K., \& Sivaganensan. (2007). The role of social support and integration for understanding socioeconomic disparities in self-rated health and hypertension. Social Science and Medicine, 65: 958-975.

Grant, B. F., \& Harford, T. C. (1995). Comorbidity between DSM-IV alcohol use disorder and major depression: Results of a National Survey. Drug and Alcohol Dependence, 39: 197-206.

Haidt, J. \& Rodin, J. (1999). Control and efficacy as interdisciplinary Bridges. Review of General Psychology, 3: 317-337.

Harman, J. S., Edlund, M. J., Fortney, J. C., \& Kallas, H. (2005). The influence of comorbid chronic medical conditions on the adequacy of depression care for older adults. Journal of the American Geriatric Society, 53: 2178-2183. 
Harris, J. M., \& Blisard, N. (2002). Food spending and the elderly. Food Review, 25: 14-18.

Hasin, D. \& Carpenter, K. M. (1998). Difficulties with questions on usual drinking and the measurement of alcohol consumption. Alcoholism: Clinical and Experimental Research, 22: 580-584.

Hastings, S. N., George, L. K., Fillenbaum, G. G., Park, R. S., Burchett, B. M., \& Schmader, K. E. (2008). Does lack of social support lead to more ED visits for older adults? American Journal of Emergency Medicine, 26: 454-461.

Hauenstein, E. J., \& Boyd, M. R. (1994). Depressive symptoms in young women of the Piedmont: Prevalence in rural women. Women and Health, 2: 105-123.

Hauenstein, E. J., \& Peddada, S. (2007). Prevalence of major depressive episodes in rural women using primary care. Journal of Health Care for the Poor and Underserved, 18: 185-202.

Howard, D. L., Bunch, C. D., Mundia, W. O., Konrad, T. R., Edwards, L. J., Amamoo, M. A., \& Jallah, Y. (2006). Comparing United States versus international medical school graduate physicians who serve African-American and White Elderly. Health Service Research, 41: 2155-2181.

Hoyt, D. R., Conger, R. D., Valde, J. G., \& Weihs, K. (1997). Psychological distress and help-seeking in rural America. American Journal of Community Psychology, 25: 449-470.

Hu, J., Amoako, E. P., Gruber, K. J., \& Rossen, E. K. (2007). The relationship among health functioning indicators and depression in older adults with diabetes. Issues in Mental Health Nursing, 28: 133-150. 
Hudson, D. L. (2009). Race, socioeconomic position and depression: The mental health cost of upward mobility. Available from the University of Michigan's Deep Blue online Dissertations and Theses: http://hdl handle net/2027.42/64820.

Hutcheson, T. D., Greiner, A., Ellerbeck, E. F., Jefferies, S. K., Mussulman, L. M., \& Casey, G. N. (2008). Understanding smoking cessation in rural communities. The Journal of Rural Health, 24: 116-124.

Idler, E.L., Benyamini, Y. (1997). Self-rated health and physical disability in elderly survivors of a major medical event. Journals of Gerontology Series BPsychological Sciences \& Social Sciences, 51, S96-104.

Jacelon, C. S. (2007). Theoretical perspectives of perceived control in older adults: a selective review of the literature. Journal of Advanced Nursing, 59: 1-10.

Jorge, R. E., Moser, D. J., Acion, L., \& Robinson, R. G. (2008). Treatment of vascular depression using repetitive transcranial magnetic stimulation. Archives of General Psychiatry, 65: 268-276.

Judge, T. A., Erez, A., Bono, J. E., \& Thoresen, C. J. (2002). Are measures of selfesteem, neuroticism, locus of control, and generalized self-efficacy indicators of a common core construct? Journal of Personality and Social Psychology, 83: 693710.

Kales, H. C., \& Valenstein, M. (2002). Complexity in late-life depression: Impact of confounding factors on diagnosing, treatment, and outcomes. Journal of Geriatric Psychiatry and Neurology, 15: 147-155.

Kane, R.A., \& Kane, R. A. (1981). Assessing the Elderly: A practical guide to measurement. Lexington, MA: Lexington Books. 
Katz, S., Ford, A.B., Moskowitz, R.W., Jackson, B.A., Jaffe, M.W. (1963). Studies of illness in the aged. The index of ADL: A standardized measure of biological and psychosocial function. Journal of the American Medical Association, 185, 914919.

Kawachi, I., Daniels, N., \& Robinson, D. E. (2005). Health disparities by race and class: Why both matter. Health Affairs, 24: 343-352.

Keller, H. H. (2006). The SCREEN I (Seniors in the Community: Risk Evaluation for Eating and Nutrition) index adequately represents nutrition risk. Journal of Clinical Epidemiology, 59: 836-841.

Keller, H. H., McKenzie, J. D., \& Goy, R. E. (2001). Construct validation and test-retest reliability of the Seniors in the Community: Risk Evaluation for Eating and Nutrition Questionnaire. Journal of Gerontology: Medical Sciences, 56A: M552M558.

Kelley-Moore, J. A., \& Ferraro, K. F. (2005). A 3-D model of health decline: Disease, disability, and depression among black and white older adults. Journal of Health and Social Behavior, 46: 376-391.

Kessler, B. C., Berglund, P., Demler, O., Jin, R., Koretz, D., Merikangas, K., R., Rush, A. J., Wlaters, E. E., \& Wang, P. S. (2003). The epidemiology of Major Depressive Disorder: Results from the National Comorbidity Survey Replication (NCS-R). JAMA, 289: 3095-3105.

Kim, J., Stewart, R., Shin, I., Yoon, J., \& Lee, H. (2004). Lifetime urban/rural residence, social support, and late-life depression in Korea. International Journal of Geriatric Psychiatry, 19: 843-851. 
Klonoff, E. A. (2009). Disparities in the provision of medical care: An outcome in search of an explanation. Journal of Behavioral Medicine, 32: 48-63.

Klugman, C. M. (2008). Vast tracts of land: Rural healthcare culture. The American Journal of Bioethics, 8: 57-71.

Koenig, H. G., \& George, L. K. (1998). Depression and physical disability in depressed medically ill hospitalized older adults. American Journal of Geriatric Psychiatry, 6: $230-247$.

Koenig, H. G., Shelp, F., Goli, V., Cohen, H. J., \& Blazer, D. G. (1989). Survival and health care utilization in elderly medical inpatients with major depression. Journal of the American Geriatric Society, 37: 599-606.

Komiti, A., Judd, F., \& Jackson, H. (2006). The influence of stigma and attitudes on seeking help from a GP for mental health problems: A rural context. Social Psychiatry in Psychiatric Epidemiology, 41: 738-745.

Kouzis, A. C., \& Eaton, W. W. (1998). Absence of social networks, social support and health services utilization. Psychological Medicine, 28: 1301-1310.

Lacro, J. P., \& Jeste, D. V. (1994). Physical comorbidity and polypharmacy in older psychiatric patients. Biological Psychiatry, 36: 146-152.

Landerman, R. (1998). EPESE composite measures and other commonly used measures: Description and documentation of original variables and variables with missing data imputed.

Landerman, R., George, L. K., Campbell, R. T., \& Balzer, D. G. (1989). Alternative models of the stress buffering hypothesis. American Journal of Community Psychology, 17: 625-642. 
Lane, J. E., Shellenberger, S., Gresen, K. W., \& Moore, N. C. (2000). Screening for depression within a rural primary care practice by a self-rating depression scale. Primary Care Psychiatry, 6: 73-76.

Lawhorne, L. (2005). Depression in the older adult. Primary Care: Clinics in Office Practice, 32: 777-792.

Lebowitz, B. D., Pearson, J. L., Schneider, L. S., Reynolds, C. F., Alexopoulos, G. S., Bruce, M. L., Conwell, Y., Katz, I. R., Meyers, B. S., Morrison, M. F., Mossey, J., Niederehe, G., \& Parmelee, P. (1997). Diagnosis and treatment of depression in late life: Consensus statement update. Journal of the American Medical Association (JAMA), 278: 1186-1190.

Lewinsohn, P. M., Seeley, J. R., Roberts, R. E., \& Allen, N. B. (1997). Center for Epidemiologic Studies Depression Scale (CES-D) as a screening instrument for depression among community-residing older adults. Psychology and Aging, 12: $277-287$.

Lindwall, M., Rennemark, M., Halling, A., Berglund, J., \& Hassmen, P. (2006). Depression and exercise in elderly men and women: Findings from the Swedish National Study on Aging and Care. Journal of Aging and Physical Activity, 15: 41-55.

Linn, J. G., \& Husaini, B. A. (1985). Chronic medical problems, coping resources, and depression: A longitudinal study of rural Tennesseans. American Journal of Community Psychology, 13: 733-742. 
Linn, L. G., Husaini, B. A., Whitten-Stovall, R., \& Broomes, R. L. (1989). Community satisfaction, life stress, social support, and mental health in rural and urban southern black communities. Journal of Community Psychology, 17: 78-88.

Lyness, J. M., Bruce, M. L., Koenig, H. G., Parmelee, P. A., Schulz, R., Lawton, M. P., \& Reynolds, C. F. (1996). Depression and medical illness in late-life: Report of a symposium. Journal of the American Geriatric Society, 44: 198-203.

Lyness, J. M., Cox, C., Curry, J., Conwell, Y., King, D. A., \& Caine, E. D. (1995). Older age and the underreporting of depressive symptoms. Journal of the American Geriatrics Society, 43: 216-221.

Lyness, J. M., King, D. A., Cox, C., Yoediono, Z., \& Caine, E. D. (1999). The importance of subsyndromal depression in older primary care patients: Prevalence and associated functional disability. Journal of the American Geriatric Society, 47: 647-652.

Mast, B. T. , MacNeill, S. E., \& Lichtenberg, P. A. (2004). Post-stroke and clinicallydefined vascular depression in geriatric rehabilitation patients. American Journal of Geriatric Psychiatry, 12: 84-92.

Mast, B. T., Neufeld, S., MacNeill, S. E., \& Lichtenberg, P. A. (2004). Longitudinal support for the relationship between vascular risk factors and late-life depressive symptoms. American Journal of Geriatric Psychiatry, 12: 93-101.

McAvay, G. J., Seeman, T. E., \& Rodin, J. (1996). A longitudinal study of change in domain-specific self-efficacy among older adults. Journal of Gerontology, PSYCHOLOGICAL SCIENCES, 51B: P243-P253. 
McCrone, S., Cotton, S., Jones, L., Hawkins, T. A., Constante, J., \& Nuss, M. (2007). Depression in a rural, free clinic, providing primary care: Prevalence and predictive factors. Archives of Psychiatry Nursing, 21: 291-293.

McDermott, R. J., Hawkins, W. E., Littlefield, E. A., \& Murray, S. (1989). Health behavior correlates of depression among university students. Journal of American College Health, 38: 115-119.

Missing value imputations for EPESE composites and questionnaire items. (1992). Study document provided by Duke EPESE investigators. Received on April 192010. Mojtabai, R. \& Olfson, M. (2004). Major Depression in community-dwelling middleaged and older adults: Prevalence and 2- and 4-year follow-up symptoms. Psychological Medicine, 34: 623-634.

Moore, M. J., Moir, P., \& Patrick, M. M. (2004). The state of aging and health in America 2004. The Merck Institute of Aging, The Centers for Disease Control, \& the Gerontological Society of America.

Muntaner, C., \& Barnett, E. (2000). Depressive symptoms in rural West Virginia: Labor market and health services correlates. Journal of Health Care for the Poor and Underserved, 11: 284-300.

Murrell, S. A., Himmelfarb, S., \& Wright, K. (1983). Prevalence of depression and its correlates in older adults. American Journal of Epidemiology, 117: 173-185.

Neff, J. A. (1983). Urbanicity and depression reconsidered: The evidence regarding depressive symptomotology. The Journal of Nervous and Mental Disease, 171: 546-552. 
Nemet. G. F. \& Bailey, A. J. (2000). Distance and health care utilization among the rural elderly. Social Science and Medicine, 50: 1197-1208.

O'Hara, M. W., Kohout, F. J., \& Wallace, R. B. (1985). Depression among the rural elderly: A study of prevalence and correlates. The Journal of Nervous and Mental Disease, 173: 582-589.

O’Neil, D. (2006). Ethnicity and Race: Overview. Retrieved June 1, 2010, from http://anthro.palomar.edu/ethnicity/ethnic_1.htm.

Onyike, C. U., Crum, R. M., Lee, H. B., Lyketsos, C. G., \& Eaton, W. W. (2003). Is obesity associated with major depression? Results from the Third National Health and Nutrition Examination Survey. American Journal of Epidemiology, 158: $1139-1147$.

Pamies R. J., \& Nsiah-Kumo, P. A. (2009). Addressing health disparities in the $21^{\text {st }}$ century. In: Cultural Proficiency in Addressing Health Disaprities. KosokoLasaki, S., Cook, C. T., \& O’Brien, R. L. (Eds.). Boston: Jones and Bartlett Publishers.

Paringer, L., Bluck, J., Feder, J., Holahan, J. (1979). Health Status and Use of Medical Services: Evidence on the Poor, the Black, and the Rural Elderly. Washington, D.C.: The Urban Institute.

Parks, C. P., \& Neutens, J. G. (1987). Poor, rural, Blacks: Health status, locus of control, and health education needs. Health Education, December 1986/January 1987: 4-8. 
Pearlin, L. I., Nguyen, K. B., Scheiman, S. \& Milkie, M. A. (2007). The life-course origins of mastery among older people. Journal of Health and Social Behavior, 48:164-179.

Pearlin, L. I., \& Schooler, C. (1978). The structure of coping. Journal of Health and Social Behavior, 19: 2-21.

Pennix, B., Kriegsman, D. M. W., van Eijk, J. T., Boeke, A. J. P., \& Deeg, D. H. (1996). Differential effects of social support on the course of chronic disease: A criteriabased literature study. Family Systems and Health, 14: 223-244.

Pennix, B., Leveille, S., Ferrucci, L., van Eijk, J., \& Guralnik, J. (1999). Exploring the effect of depression on physical disability: Longitudinal evidence from the Established Populations for Epidemiologic Studies of the Elderly. American Journal of Public Health, 89: 1346-1352

Pilisuk, M., Boylan, R., \& Acredolo, C. (1987). Social support, life stress, and subsequent medical care utilization. Health Psychology, 6: 273-288.

Potts, M. K. (1997). Social support and depression among older adults living alone: The importance of friends within and outside of a retirement community. Social Work, 42: 348-362.

Probst, J. C ., Laditka, S. B., Moore, C. G., Harun, N., Powell, M. P., \& Baxley, E. G. (2006). Rural-urban differences in depression prevalence: Implications for family medicine. Family Medicine, 38: 653-660.

Radloff, L. S. 1977. The CES-D Scale: A self-report depression scale for research in the general population. Applied Psychological Measurement 1: 385-401. 
Redwood, Y., Schulz, A. J., Israel, B. A., Yoshihama, M., Wang, C. C., \& Kreuter, M. (2010). Social, economic, and political processes that create built environment inequities: Perspectives from urban African Americans in Atlanta. Community Health, 33: 53-67.

Rokke, P. D. \& Klenow, D. J. (1998). Prevalence of depressive symptoms among rural elderly: Examining the need for mental health services. Psychotherapy, 35: 545558.

Rural Population and Migration: Trend 6-Challenges from an Aging Population. 2007. United States Department of Agriculture. Economic Research Service: The Economics of Food, Farming, Natural Resources, and Rural America. http://www.ers.usda.gov/Briefing/Population/Challenges.htm. Retrieved: July 27, 2009.

Russell, R. M., Rasmussen, H., \& Lichtenstein, A. H. (1999). Modified food guide pyramid for people over seventy years of age. The Journal of Nutrition, 129: 751753.

Ruthig, J. C., Chipperfield, J. G., Bailis, D. S., \& Perry, R. P. (2008). Perceived control and risk characteristics as predictors of older adult's health risk estimated. The Journal of Social Psychology, 148: 667-688.

Scheidt, R. J. (1984). The mental health of the aged in rural environments. In R. T. Coward and G. R. Lee (Eds.), The Elderly in Rural Society (p. 105-127). New York: Springer Publishing. 
Schulman, E., Gairola, G., Kuder, L., \& McCulloch, J. (2002). Depression and associated characteristics among community-based elderly people. Journal of Allied Health, 31: 140-146.

Schwab, J. J., Warheit, G. J., \& Holzer, C. E., III. (1974). Mental health: Rural-urban comparisons. Mental Health Society, 1: 265-274.

Sears, S. F., Danda, C. E., \& Evans, G. D. (1999). PRIME-MD and rural primary care: Detecting depression in a low-income rural population. Professional Psychology, Research and Practice, 30: 357-360.

Shear, K., Ginsberg, D. L., Roose, S. P., Lenze, E. J., Alexopoulos, G. S., \& Hollander, E. (2005). Depression in the elderly: The unique features related to diagnosis and treatment. CNS Spectrums, 10: A1-A13.

Simmons, L. A., \& Havens, J. R. (2007). Comorbid substance and mental disorders among rural Americans: Results from the national comorbidity survey. Journal of Affective Disorders, 99: 265-271.

Simmons, L. A., Huddleston-Casas, C., \& Berry, A. A. (2007). Low-income rural women and depression: Factors associated with self-reporting. American Journal of Health Behavior, 31: 657-666.

Simon, G. E., Von Korff, M., Saunders, K., Miglioretti, D. L., Crane, P. K., Van Belle, G., \& Kessler, R. C. (2006). Association between obesity and psyhcoatric disorders in the US adult population. Archives of General Psychiatry, 63: 824830. 
Sirey, J. A., Raue, P. J., \& Alexopoulos, G. S. (2007). An intervention to improve depression care in older adults with COPD. International Journal of Geriatric Psychiatry, 22: 154-159.

Slagsvold, B. \& Sorensen, A. (2008). Age, education, and the gender gap in the sense of control. International Journal of Aging and Human Development, 67: 25-42.

Spotila, J. T. (2000). Standards for defining metropolitan and micropolitan statistical Areas. (Federal Record No. 00-32997, pp. 82228-82238). Washington, DC: US Office of Management and Budget.

Stage, K. B., Middelboe, K. B., \& Pisinger, C. (2005). Depression and chronic obstructive pulmonary disease (COPD). Impact on survival. Acta Psychiatrica Scandinavica, 111: 320-323.

Stamm, B. H., Lambert, D., Piland, N. F., \& Speck, N. C. (2007). A rural perspective on health care for the whole person. Professional Psychology: Research and Practice, 38: 298-304.

Stevens, K. R., \& Munoz, L. R. (2004). Cigarette smoking: evidence to guide measurement. Research in Nursing and Health, 27: 281-292.

St John, P. D., Blandford, A. A., \& Strain, L. A. (2002). Depressive symptoms among older adults in urban and rural areas. International Journal of Geriatric Psychiatry, 21: 1175-1180.

Strain, L. A. (1991). Use of health services in later life: The influence of health beliefs. Journal of Gerontology, 46: S143-S150.

Swenson, C. J., Baxter, J., Shetterly, S. M., Scarbro, S. L., \& Hamman, R. F. (2000). Depressive symptoms in Hispanic and Non-Hispanic White rural elderly: The San 
Luis Valley Health and Aging Study. American Journal of Epidemiology, 152: 1048-1055.

Thomas, D. C. (1999). Primary care for people with disabilities. The Mount Sinai Journal of Medicine, 66: 188-191.

Thorson, J. A., \& Powell, F. C. (2000). Health Practices and attitudes among rural, semi-rural, and urban older people. North American Journal of Psychology, 2: $47-58$.

Turner, R. J., \& Noh, S. (1988). Physical disability and depression: A longitudinal analysis. Journal of Health and Social Behavior, 29: 23-37.

Understanding Health Disparities. (2004). The Health Policy Institute of Ohio, Columbus Ohio: 1-32.

VanderMey Barr, D. (2007). Oregon WIC Training Risk Module. Oregon Department of Human Services. Document retrieved on August 5, 2009 from: http://www.oregon.gov/DHS/ph/wic/docs/NR training module all.pdf.

Veltman, A., Stewart, D. E., Tardif, G. S., \& Branigan, M. (2001). Healthcare for people with physical disabilities - Access issues: Results. Medscape Today, 3 .

Vitolins, M. Z., Tooze, J. A., Golden, S. L., Arcury, T. A., Bell, R. A., Davis, C., Devellis, R. F., \& Quandt, S. A. (2007). Older adults in the rural south are not meeting healthful eating guidelines. Journal of the American Dietetic Association, 107: 265-272.

Voaklander, D., C., Thommasen, H., V., \& Michalos, A. C. (2006). The relationship between health survey and medical chart review results in a rural population. Social Indicators Research, 77: 287-305. 
Wallace, J. \& O'Hara, M. W. (1992). Increases in depressive symptomatology in the rural elderly: Results from a cross-sectional and longitudinal study. Journal of Abnormal Psychology, 101: 398-404.

Washington State Department of Health. Guidelines for using rural-urban classification systems for public health assessment. Retrieved March, 20, 2009, from http://www.doh.wa.gov/Data/Guidelines/RuralUrban.htm.

Wei, T., Zeng, C., Chen, L., Wang, S., Li, S., Chen, Q., \& Wang, L. (2004). Predicting factors of depression in patients with primary hypertension: a community-based study. International Journal of Psychiatry in Clinical Practice, 8: 213-217.

Williams, D. R., \& Mohamed, S. (2009). Discrimination and racial disparities in health: evidence and needed research. Journal of Behavioral Medicine, 32: 20-47.

Wolinsky, F. D., Wyrwich, K. W., Babu, A. N., Kroenke, K., \& Tierney, W. M. (2003). Age, aging, and the sense of control among older adults: A longitudinal reconsideration. Journal of Gerontology, SOCIAL SCIENCES, 58B, S212-S220. Wrigley, S. Jackson, H., Judd, F., \& Komiti, A. (2005). Role of stigma and attitudes toward help-seeking from a general practitioner for mental health problems in a rural town. Australian and New Zealand Journal of Psychiatry, 39: 514-521.

Yochim, B. P., Kerkar, S. P., \& Lichtenberg, P. A. (2006). Cerebrovascular risk factors, activity limitations, and depressed mood in African American older adults. Psychology and Aging, 21: 186-189.

Zimmerman, J. A., Mast, B. T., Miles, T., \& Markides, K. S. (2009). Vascular risk and depression in the Hispanic Established Population for the Epidemiologic Study of the Elderly (EPESE). International Journal of Geriatric Psychiatry, 24: 409-416. 
Zulkowski, K., \& Coon, P. J. (2004). Comparison of nutritional risk between urban and rural elderly. Ostomy/Wound Management, 50: 46-58. 
APPENDICES 


\section{Appendix A: List of Tables}

Table 1

Description of health disparities investigated

\begin{tabular}{|l|l|}
\hline \multicolumn{1}{|c|}{ Health Disparity } & \multicolumn{1}{c|}{ Description } \\
\hline $\begin{array}{l}\text { Healthcare utilization (HCU) - contact } \\
\text { with health care providers }\end{array}$ & $\begin{array}{l}\text { Number of outpatient medical visits (to } \\
\text { physician, clinic, emergency department) in } \\
\text { the past 12 months. }\end{array}$ \\
\hline $\begin{array}{l}\text { Overall medical burden - number of } \\
\text { chronic medical conditions }\end{array}$ & $\begin{array}{l}\text { Chronic disease conditions reported by the } \\
\text { participant (e.g. diabetes, high blood } \\
\text { pressure, heart disease, etc). }\end{array}$ \\
\hline $\begin{array}{l}\text { Cumulative vascular risk (CVR) - number } \\
\text { of VRFs }\end{array}$ & $\begin{array}{l}\text { Vascular risk factors reported by the } \\
\text { participant (e.g. smoking, stroke, HTN, } \\
\text { diabetes, etc.). }\end{array}$ \\
\hline Negative health risk behaviors (NHBs) & $\begin{array}{l}\text { Significant daily smoking, heavy alcohol } \\
\text { consumption, and lack of physical exercise. }\end{array}$ \\
\hline $\begin{array}{l}\text { Cultural beliefs possibly related to health } \\
\text { behaviors }\end{array}$ & $\begin{array}{l}\text { Negative attitudes towards medical } \\
\text { doctors; Self-efficacy. }\end{array}$ \\
\hline
\end{tabular}


Table 2

Rural/Urban Continuum Codes by Duke EPESE county

\begin{tabular}{|c|c|c|c|}
\hline County & 1983 Code & 1993 Code & 2003 Code \\
\hline Durham & $\begin{array}{c}\mathbf{2} \\
\text { County in metro area of } \\
250,000 \text { to } 1 \text { million } \\
\text { population }\end{array}$ & $\begin{array}{c}\mathbf{2} \\
\text { County in metro area of } \\
250,000 \text { to } 1 \text { million } \\
\text { population }\end{array}$ & $\begin{array}{c}\mathbf{2} \\
\text { County in metro area of } \\
250,000 \text { to } 1 \text { million } \\
\text { population }\end{array}$ \\
\hline Franklin & $\begin{array}{c}\mathbf{2} \\
\text { County in metro area of } \\
250,000 \text { to } 1 \text { million } \\
\text { population }\end{array}$ & $\begin{array}{c}\mathbf{2} \\
\text { County in metro area of } \\
250,000 \text { to } 1 \text { million } \\
\text { population }\end{array}$ & $\begin{array}{c}\mathbf{2} \\
\text { County in metro area of } \\
250,000 \text { to } 1 \text { million } \\
\text { population }\end{array}$ \\
\hline Granville & $\begin{array}{c}\mathbf{6} \\
\text { Nonmetro county with } \\
\text { urban population of } 2,500- \\
19,999, \text { adjacent to a metro } \\
\text { area } \\
\end{array}$ & $\begin{array}{c}6 \\
\text { Nonmetro county with } \\
\text { urban population of } \\
2,500-19,999, \text { adjacent to } \\
\text { a metro area } \\
\end{array}$ & $\begin{array}{c}\mathbf{6} \\
\text { Nonmetro county with } \\
\text { urban population of } \\
2,500-19,999, \text { adjacent to } \\
\text { a metro area } \\
\end{array}$ \\
\hline Vance & $\begin{array}{c}7 \\
\text { Nonmetro county with } \\
\text { urban population of } 2,500- \\
19,999, \text { not adjacent to a } \\
\text { metro area }\end{array}$ & $\begin{array}{c}\mathbf{6} \\
\text { Nonmetro county with } \\
\text { urban population of } \\
2,500-19,999, \text { adjacent to } \\
\text { a metro area }\end{array}$ & $\begin{array}{c}\mathbf{4} \\
\text { Nonmetro county with } \\
\text { urban population of } \\
20,000 \text { or more, adjacent } \\
\text { to a metro area }\end{array}$ \\
\hline Warren & $\begin{array}{c}9 \\
\text { Nonmetro county } \\
\text { completely rural or less } \\
\text { than } 2,500 \text { urban } \\
\text { population, not adj. to } \\
\text { metro area }\end{array}$ & $\begin{array}{c}\mathbf{8} \\
\text { Nonmetro county } \\
\text { completely rural or less } \\
\text { than } 2,500 \text { urban } \\
\text { population, adj. to metro } \\
\text { area }\end{array}$ & $\begin{array}{c}\mathbf{8} \\
\text { Nonmetro county } \\
\text { completely rural or less } \\
\text { than } 2,500 \text { urban } \\
\text { population, adj. to metro } \\
\text { area }\end{array}$ \\
\hline
\end{tabular}


Table 3

Description of the ERS Rural/Urban Continuum Codes (2003)

\begin{tabular}{|c|c|}
\hline Code & Description \\
\hline \multicolumn{2}{|c|}{ Metro Counties } \\
\hline 1 & Counties in metro areas, population $>1$ million \\
\hline 2 & Counties in metro areas, population $250,000-1$ million \\
\hline 3 & Counties in metro areas, population $<250,000$ \\
\hline \multicolumn{2}{|c|}{ Nonmetro Counties } \\
\hline 4 & Urban population $>20,000$, adjacent to metro area \\
\hline 5 & Urban population $>20,000$, not adjacent to metro area \\
\hline 6 & Urban population, $2,500-19,000$, adjacent to metro area \\
\hline 7 & Urban population, $2,500-19,000$, not adjacent to metro area \\
\hline 8 & $\begin{array}{c}\text { Completely Rural, or less than } 2,500 \text { urban population, } \\
\text { adjacent to a metro area }\end{array}$ \\
\hline 9 & $\begin{array}{l}\text { Completely Rural, or less than } 2,500 \text { urban population, not } \\
\text { adjacent to a metro area }\end{array}$ \\
\hline
\end{tabular}

Adapted from The United States Department of Agriculture Website: http://www.ers.usda.gov/briefing/rurality/RuralUrbCon/ 
Table 4

Demographic characteristics of county codes - Wave $1(\mathrm{~N}=3,996)$

\begin{tabular}{|c|c|c|c|c|c|}
\hline Characteristic & Code 2 & Code 6 & Code 7 & Code 9 & Total \\
\hline Counties & $\begin{array}{l}\text { Durham } \\
\text { Franklin }\end{array}$ & Granville & Vance & Warren & ----- \\
\hline $\mathrm{n}$ in Code & $2437^{\mathrm{a}}$ & 527 & 628 & 404 & 3,996 \\
\hline \multicolumn{6}{|l|}{ *Age (years) } \\
\hline Mean (SD) & $73.41(6.58)$ & $72.88(6.40)$ & $72.83(6.01)$ & $73.94(6.93)$ & $73.30(6.51)$ \\
\hline \multicolumn{6}{|l|}{ Gender $(\%)$} \\
\hline Male & $34.14 \%$ & $36.05 \%$ & $37.73 \%$ & $33.91 \%$ & $34.93 \%$ \\
\hline \multicolumn{6}{|l|}{$*$ Race $(\%)$} \\
\hline \multicolumn{6}{|c|}{ Non-Hispanic- } \\
\hline $\begin{array}{l}\text { White } \\
\text { Black }\end{array}$ & $\begin{array}{l}50.18 \% \\
49.12 \%\end{array}$ & $\begin{array}{l}42.69 \% \\
57.12 \%\end{array}$ & $\begin{array}{l}42.99 \% \\
57.01 \%\end{array}$ & $\begin{array}{l}25.00 \% \\
73.51 \%\end{array}$ & $\begin{array}{l}45.52 \% \\
53.88 \%\end{array}$ \\
\hline Other & $<1.00 \%$ & $<1.00 \%$ & $0.00 \%$ & $1.49 \%$ & $0.60 \%$ \\
\hline \multicolumn{6}{|l|}{ *Yearly Income } \\
\hline $\begin{array}{l}\text { Mean } \\
\text { (SD) }\end{array}$ & $\begin{array}{l}11523.15 \\
(10734.38)\end{array}$ & $\begin{array}{l}8451.75 \\
(8189.83)\end{array}$ & $\begin{array}{l}9107.61 \\
(8760.99)\end{array}$ & $\begin{array}{l}7377.58 \\
(7418.32)\end{array}$ & $\begin{array}{l}10319.35 \\
(9956.91)\end{array}$ \\
\hline \multicolumn{6}{|l|}{ *Education } \\
\hline Mean (SD) & $8.84(4.15)$ & $8.20(3.66)$ & $7.91(4.02)$ & $8.21(4.03)$ & $8.55(4.07)$ \\
\hline \multicolumn{6}{|l|}{ Marital Status (\%) } \\
\hline Currently married & $37.92 \%$ & $40.42 \%$ & $38.06 \%$ & $41.58 \%$ & $38.64 \%$ \\
\hline \multicolumn{6}{|c|}{ **Employment Status (\%) } \\
\hline Currently Working & $13.55 \%$ & $13.09 \%$ & $9.08 \%$ & $8.66 \%$ & $12.29 \%$ \\
\hline \multicolumn{6}{|l|}{ CES-D score } \\
\hline Mean (SD) & $3.18(3.53)$ & $3.22(3.25)$ & $3.33(3.33)$ & $3.53(3.11)$ & $3.24(3.42)$ \\
\hline CES-D $\geq 9(\%)$ & $10.14 \%$ & $9.11 \%$ & $8.44 \%$ & $8.91 \%$ & $9.6 \%$ \\
\hline
\end{tabular}

${ }^{a}$ : Durham $(\mathrm{n}=1928) ;$ Franklin $(\mathrm{n}=509)$

$* \mathrm{p}<0.05 ;{ }^{* *} \mathrm{p}<0.01$ 
Table 5

Association between study demographic variables and depression (mean CES-D score)

\begin{tabular}{|l|l|l|}
\hline Demographic Variable & Level - Mean CES-D score $(\mathrm{n})$ & ANOVA results \\
\hline Race & $\begin{array}{l}\text { Non-Hispanic White }=3.04(\mathrm{n}=1819) \\
\text { Black }=3.41(\mathrm{n}=2153)\end{array}$ & $\begin{array}{l}\mathrm{F}(18,3953)=3.38, \\
\mathrm{p}<0.001\end{array}$ \\
\hline Sex & $\begin{array}{l}\text { Male }=2.68(\mathrm{n}=1396) \\
\text { Female }=3.55(\mathrm{n}=2600)\end{array}$ & $\begin{array}{l}\mathrm{F}(18,3977)=5.02, \\
\mathrm{p}<0.001\end{array}$ \\
\hline Age & $\begin{array}{l}64-74=3.04(\mathrm{n}=2499) \\
75-84=3.53(\mathrm{n}=1230) \\
85+=3.82(\mathrm{n}=267)\end{array}$ & $\begin{array}{l}\mathrm{F}(2,3993)=12.41, \\
\mathrm{p}<0.001\end{array}$ \\
\hline $\begin{array}{l}\text { Education } \\
\text { (years completed) }\end{array}$ & $\begin{array}{l}0-5=3.98(\mathrm{n}=965) \\
6-8=3.55(\mathrm{n}=1132) \\
9-11=3.02(\mathrm{n}=984)\end{array}$ & $\begin{array}{l}\mathrm{F}(18,3977)=8.97, \\
\mathrm{p}<0.001\end{array}$ \\
\hline $\begin{array}{l}\text { Annual Income } \\
\text { (in U.S. dollars) }\end{array}$ & $\begin{array}{l}999.5-3499.5=4.15(\mathrm{n}=792) \\
4499.5-5999.5=3.69(\mathrm{n}=1473) \\
8499.5-12499.5=2.73(\mathrm{n}=977) \\
17499.5-44529.8=2.09(\mathrm{n}=754)\end{array}$ & $\begin{array}{l}\mathrm{F}(18,3977)=14.69, \\
\mathrm{p}<0.001\end{array}$ \\
\hline Marital Status & $\begin{array}{l}\text { Married }=2.63(\mathrm{n}=1544) \\
\text { Not Married }=3.63(\mathrm{n}=2452)\end{array}$ & $\begin{array}{l}\mathrm{F}(18,3977)=6.23, \\
\mathrm{p}<0.001\end{array}$ \\
\hline Work Status & $\begin{array}{l}\text { Currently Working }=2.10(\mathrm{n}=491) \\
\text { Not Working }=3.40(\mathrm{n}=3504)\end{array}$ & $\begin{array}{l}\mathrm{F}(18,3976)=4.61, \\
\mathrm{p}<0.001\end{array}$ \\
\hline
\end{tabular}


Table 6

Percent of participants by level of HDs and Mean (SD) of HD by total sample and county code

\begin{tabular}{|c|c|c|c|c|c|}
\hline Health Disparity & Code $2(\%)$ & Code $6(\%)$ & Code $7(\%)$ & Code $9(\%)$ & $\begin{array}{c}\text { Total } \\
\text { Sample }\end{array}$ \\
\hline $\begin{array}{l}\text { Overall Medical } \\
\text { Burden }\end{array}$ & \multicolumn{4}{|c|}{$\chi^{2}(9)=8.35, p=0.50$} & \\
\hline 0 conditions & 25.4 & 22.7 & 23.0 & 24.3 & 21.4 \\
\hline 1 condition & 36.4 & 38.9 & 39.6 & 37.4 & 37.2 \\
\hline 2 conditions & 24.5 & 25.7 & 25.1 & 24.7 & 24.6 \\
\hline $3+$ conditions & 13.7 & 12.8 & 12.2 & 16.1 & 13.5 \\
\hline $\begin{array}{l}\text { Mean (SD) \# of } \\
\text { conditions }\end{array}$ & $1.27(0.99)$ & $1.29(0.96)$ & $1.27(0.95)$ & $1.34(0.99)$ & $\begin{array}{l}1.28 \\
(0.98)\end{array}$ \\
\hline HCU & \multicolumn{4}{|c|}{$\chi^{2}(12)=32.78, \mathrm{p}<0.01$} & \\
\hline 0 visits & 20.5 & 19.2 & 18.6 & 19.3 & 18.5 \\
\hline $1-3$ visits & 37.0 & 33.8 & 37.4 & 36.1 & 34.6 \\
\hline 4-6 visits & 21.8 & 26.5 & 25.1 & 23.8 & 22.8 \\
\hline $7-12$ visits & 13.9 & 13.8 & 14.4 & 17.4 & 13.7 \\
\hline$>12$ visits & 6.9 & 6.5 & 4.4 & 6.7 & 6.2 \\
\hline $\begin{array}{l}\text { Mean (SD) \# of } \\
\text { visits }\end{array}$ & $1.50(1.16)$ & $1.55(1.14)$ & $1.49(1.09)$ & $1.72(1.11)$ & $\begin{array}{l}1.52 \\
(1.14) \\
\end{array}$ \\
\hline CVR & \multicolumn{4}{|c|}{$\chi^{2}(9)=9.68, p=0.38$} & \\
\hline 0 conditions & 15.1 & 12.4 & 13.6 & 15.2 & 14.4 \\
\hline 1 condition & 35.3 & 35.0 & 36.5 & 36.2 & 35.5 \\
\hline 2 conditions & 30.4 & 32.8 & 31.0 & 25.7 & 30.3 \\
\hline $3+$ conditions & 19.2 & 19.8 & 18.9 & 22.9 & 19.6 \\
\hline $\begin{array}{l}\text { Mean (SD) \# of } \\
\text { conditions }\end{array}$ & $1.54(0.97)$ & $1.60(0.94)$ & $1.55(0.95)$ & $1.56(1.01)$ & $\begin{array}{l}1.55 \\
(0.96) \\
\end{array}$ \\
\hline Health Disparity & Code $2(\%)$ & Code $6(\%)$ & Code $8(\%)$ & 一 & \\
\hline $\mathrm{NHB}^{\mathbf{a}}$ & \multicolumn{4}{|c|}{$\chi^{2}(4)=9.47, p=0.05$} & \\
\hline $0 \mathrm{NHBs}$ & 19.8 & 17.2 & 18.1 & & 17.8 \\
\hline $1 \mathrm{NHB}$ & 52.7 & 51.9 & 53.1 & & 49.9 \\
\hline $2+\mathrm{NHBs}$ & 27.5 & 31.0 & 28.0 & & 26.3 \\
\hline $\begin{array}{l}\text { Mean (SD) \# of } \\
\text { behaviors }\end{array}$ & $1.08(0.68)$ & $1.17(0.69)$ & $1.05(0.64)$ & & $\begin{array}{l}1.09 \\
(0.68)\end{array}$ \\
\hline
\end{tabular}

$\mathrm{a}=$ Wave 2 county codes ( 3 levels) and Wave 2 Total Sample 
Table 7

Follow-up post hoc analyses: Healthcare utilization and county code

\begin{tabular}{|c|c|c|c|c|c|}
\hline $\begin{array}{c}\text { Comparison } \\
(\mathrm{HCU})\end{array}$ & Chi-square & $\begin{array}{c}\text { Significance } \\
\text { Level }\end{array}$ & & & \\
\hline 2 vs 6 & $\chi^{2}(4)=5.82$ & $p=0.21$ & & & \\
\hline 2 vs 7 & $\chi^{2}(4)=8.07$ & $\mathrm{p}=0.09$ & & & \\
\hline 2 vs 9 & $\chi^{2}(4)=24.29$ & $p<0.001$ & & & \\
\hline 6 vs 7 & $\chi^{2}(4)=3.61$ & $\mathrm{p}=0.45$ & & & \\
\hline 6 vs 9 & $\chi^{2}(4)=7.38$ & $p=0.12$ & & & \\
\hline 7 vs 9 & $\chi^{2}(4)=11.79$ & $\mathrm{p}=0.02$ & & & \\
\hline Code & 0 visits & 1-3 Visits & 4-6 Visits & 7-12 Visits & 13+ Visits \\
\hline 2 & 20.5 & 37.0 & 21.8 & 13.9 & 6.9 \\
\hline 9 & 19.3 & 36.1 & 23.8 & 17.4 & 6.7 \\
\hline
\end{tabular}


Table 8

Place of usual healthcare by county code: private practice, public clinic, and nowhere

\begin{tabular}{|l|l|l|l|}
\hline County Code & Private Practice (\%) & Public Clinic (\%) & Nowhere (\%) \\
\hline 2 & 63.3 & 11.4 & 2.0 \\
\hline 6 & 79.8 & 6.7 & 2.5 \\
\hline 7 & 84.4 & 6.7 & 0.6 \\
\hline 9 & 52.4 & 35.7 & 4.2 \\
\hline
\end{tabular}


Table 9

Differences between participants who use private practice offices vs. public clinics

\begin{tabular}{|l|l|l|}
\hline & Private Practice (\%) & Public Clinic (\%) \\
\hline Overall medical burden & & \\
\hline 0 conditions & 24.6 & 14.3 \\
\hline 1 condition & 38.5 & 37.3 \\
\hline 2 conditions & 24.7 & 27.6 \\
\hline $3+$ conditions & 12.2 & 20.8 \\
\hline & & \\
\hline HCU & & \\
\hline 0 visits & 17.6 & 7.7 \\
\hline $1-3$ visits & 36.2 & 35.8 \\
\hline $4-6$ visits & 24.3 & 34.3 \\
\hline $7-12$ visits & 15.1 & 16.0 \\
\hline$>12$ visits & 6.8 & 6.2 \\
\hline & & \\
\hline Satisfaction with healthcare & & \\
\hline Very dissatisfied & 3.8 & 5.5 \\
\hline Dissatisfied & 4.6 & 4.5 \\
\hline Satisfied & 53.1 & 55.0 \\
\hline Very satisfied & 38.5 & 36.3 \\
\hline
\end{tabular}


Table 10

Satisfaction with healthcare services by county code

\begin{tabular}{|l|l|l|l|l|}
\hline & \multicolumn{1}{|c|}{$\begin{array}{c}\text { Very Dissatisfied } \\
\%(\mathrm{n})\end{array}$} & \multicolumn{1}{|c|}{$\begin{array}{c}\text { Dissatisfied } \\
\%(\mathrm{n})\end{array}$} & \multicolumn{1}{|c|}{$\begin{array}{c}\text { Satisfied } \\
\%(\mathrm{n})\end{array}$} & $\begin{array}{c}\text { Very Satisfied } \\
\%(\mathrm{n})\end{array}$ \\
\hline Code 2 & $3.1(74)$ & $4.4(104)$ & $50.3(1194)$ & $42.2(1001)$ \\
\hline Code 6 & $2.3(12)$ & $5.9(31)$ & $57.1(298)$ & $34.7(181)$ \\
\hline Code 7 & $3.8(23)$ & $6.1(37)$ & $67.8(410)$ & $22.3(135)$ \\
\hline Code 9 & $10.5(42)$ & $3.8(15)$ & $64.4(257)$ & $21.3(85)$ \\
\hline
\end{tabular}


Table 11

Differences on HCU between county codes 2 and 9 Blacks and Non-Hispanic Whites

County Code 2 (most urban)

\begin{tabular}{|l|l|l|}
\hline & Non-Hispanic White (\%) & Black (\%) \\
\hline HCU & & \\
\hline 0 visits & 21.4 & 19.8 \\
\hline $1-3$ visits & 39.3 & 34.2 \\
\hline $4-6$ visits & 19.7 & 23.9 \\
\hline $7-12$ visits & 12.9 & 15.0 \\
\hline$>12$ visits & 6.7 & 7.1 \\
\hline & & \\
\hline Overall Medical Burden & & \\
\hline 0 conditions & 29.0 & 21.9 \\
\hline 1 condition & 35.9 & 36.9 \\
\hline 2 conditions & 23.3 & 25.5 \\
\hline $3+$ conditions & 11.8 & 15.6 \\
\hline & & \\
\hline Satisfaction with healthcare & & \\
\hline Very Dissatisfied & 3.8 & 2.5 \\
\hline Dissatisfied & 4.8 & 3.9 \\
\hline Satisfied & 43.8 & 56.7 \\
\hline Very Satisfied & 47.6 & 36.9 \\
\hline
\end{tabular}

County Code 9 (most rural)

\begin{tabular}{|l|l|l|}
\hline & Non-Hispanic White (\%) & Black (\%) \\
\hline HCU & & \\
\hline 0 visits & 16.3 & 13.1 \\
\hline $1-3$ visits & 29.6 & 31.9 \\
\hline $4-6$ visits & 30.6 & 30.5 \\
\hline $7-12$ visits & 18.4 & 17.0 \\
\hline$>12$ visits & 5.1 & 7.4 \\
\hline & & \\
\hline Overall Medical Burden & & \\
\hline 0 conditions & 17.8 & 23.0 \\
\hline 1 condition & 43.6 & 36.1 \\
\hline 2 conditions & 26.7 & 23.3 \\
\hline $3+$ conditions & 11.9 & 17.6 \\
\hline & & \\
\hline Satisfaction with healthcare & & \\
\hline Very Dissatisfied & 13.0 & 8.9 \\
\hline Dissatisfied & 3.0 & 4.1 \\
\hline Satisfied & 56.0 & 67.6 \\
\hline Very Satisfied & 28.0 & 19.5 \\
\hline
\end{tabular}


Table 12

Percentage of Blacks and Non-Hispanic Whites by place of usual outpatient healthcare

\begin{tabular}{|l|l|l|l|l|l|}
\hline & \multicolumn{2}{|c|}{ Non-Hispanic White } & \multicolumn{3}{c|}{ Black } \\
\hline $\begin{array}{c}\text { County } \\
\text { Code }\end{array}$ & $\begin{array}{c}\text { \% Private } \\
\text { Practice }\end{array}$ & $\begin{array}{c}\text { \% Public } \\
\text { Clinic }\end{array}$ & $\begin{array}{c}\text { \% Private } \\
\text { Practice }\end{array}$ & $\begin{array}{c}\text { \% Public } \\
\text { Clinic }\end{array}$ \\
\hline 2 & 80.97 & 2.95 & & 45.81 & 19.93 \\
\hline 6 & 83.49 & 3.57 & & 77.33 & 8.67 \\
\hline 7 & 93.70 & 2.22 & & 77.37 & 10.03 \\
\hline 9 & 72.28 & 18.81 & 44.93 & 41.89 \\
\hline
\end{tabular}


Table 13

Percent of Black and Non-Hispanic White residents with prevalent depression (CES-D > 9) by county code

\begin{tabular}{|l|l|l|}
\hline \multicolumn{1}{|c|}{ County Code } & Non-Hispanic White (\%) & \multicolumn{1}{c|}{ Black (\%) } \\
\hline 2 & 9.08 & 11.28 \\
\hline 6 & 9.78 & 8.64 \\
\hline 7 & 9.63 & 7.54 \\
\hline 9 & 13.86 & 7.41 \\
\hline
\end{tabular}


Table 14

Perceived social support, overall medical burden, county code, and depression

\begin{tabular}{|c|c|c|c|}
\hline Health Disparity & $\begin{array}{c}\text { Test of } \\
\text { Interaction }\end{array}$ & Significance & Mean CES-D Score \\
\hline $\begin{array}{l}\text { Perceived Social Support } \\
\text { (PSS)(Main Effect) }\end{array}$ & $F(3,3882)=16.88$ & $\mathrm{p}<0.001$ & $\begin{array}{l}\text { Low } \mathrm{PSS}=4.97 \\
\text { Moderate PSS }=4.10 \\
\text { high } \mathrm{PSS}=3.66 \\
\text { very high PSS }=2.99\end{array}$ \\
\hline $\begin{array}{l}\text { Overall Medical Burden (\# } \\
\text { of chronic medical } \\
\text { conditions) (Main Effect) }\end{array}$ & $\mathrm{F}(3,3882)=12.34$ & $\mathrm{p}<0.001$ & $\begin{array}{l}0 \text { conditions }=2.96 \\
1 \text { condition }=3.52 \\
2 \text { conditions }=4.47 \\
3+\text { conditions }=4.78\end{array}$ \\
\hline $\begin{array}{l}\text { PSS X OMB } \\
\text { (2-Way Interaction) }\end{array}$ & $\mathrm{F}(9,3882=0.85$ & $p=0.57$ & \\
\hline $\begin{array}{l}\text { PSS X county code } \\
\text { (2-Way Interaction) }\end{array}$ & $\mathrm{F}(9,3882)=1.23$ & $p=0.28$ & \\
\hline $\begin{array}{l}\text { PSS X OMB X County Code } \\
\text { (3-Way Interaction) }\end{array}$ & $F(27,3882)=0.93$ & $\mathrm{p}=0.57$ & \\
\hline
\end{tabular}


Table 15

Perceived social support, healthcare utilization, county code, and depression

\begin{tabular}{|l|l|l|l|}
\hline Health Disparity & Test of Interaction & Significance & Mean CES-D Score \\
\hline $\begin{array}{l}\text { Perceived Social Support } \\
\text { (PSS)(Main Effect) }\end{array}$ & $\mathrm{F}(3,3731)=22.17$ & $\mathrm{p}<0.001$ & $\begin{array}{l}\text { Low PSS }=5.41 \\
\text { Moderate PSS }= \\
4.42 \\
\text { high PSS }=3.81 \\
\text { very high PSS } \\
3.06\end{array}$ \\
\hline $\begin{array}{l}\text { Healthcare Utilization } \\
\text { (\# of outpatient healthcare visi } \\
\text { past year)(Main Effect) }\end{array}$ & $\mathrm{F}(4,3731)=18.00$ & $\mathrm{p}<0.001$ & $\begin{array}{l}0 \text { visits }=3.08 \\
1-3 \text { visits }=3.04 \\
4-6 \text { visits }=4.26 \\
7-12 \text { visits }=4.61 \\
>12 \text { visits }=5.84\end{array}$ \\
\hline $\begin{array}{l}\text { PSS X HCU } \\
\text { (2-Way Interaction) }\end{array}$ & $\mathrm{F}(12,3725)=1.38$ & $\mathrm{p}=0.17$ & $\mathrm{p}=0.84$ \\
\hline $\begin{array}{l}\text { PSS X County Code } \\
\text { (2-Way Interaction) }\end{array}$ & $\mathrm{F}(9,3725)=0.54$ & $\mathrm{~F}=0.47$ & \\
\hline $\begin{array}{l}\text { PSS X HCU X County Code } \\
\text { (3-Way Interaction) }\end{array}$ & $\mathrm{F}(36,3725)=0.997$ & $\mathrm{p}=0.47$ \\
\hline
\end{tabular}


Table 16

Percentage of Blacks and Non-Hispanic Whites reporting low perceived social support ( score $=2$ or 3 ) by county code

\begin{tabular}{|l|l|l|}
\hline County Code & Non-Hispanic White (\%) & Black (\%) \\
\hline 2 & 2.79 & 6.67 \\
\hline 6 & 1.33 & 4.67 \\
\hline 7 & 3.72 & 3.08 \\
\hline 9 & 3.96 & 5.07 \\
\hline
\end{tabular}


Table 17

Physical disability (PD), overall medical burden, county code, and depression

\begin{tabular}{|l|l|l|l|}
\hline \multicolumn{1}{|c|}{ Health Disparity } & Test of Interaction & Significance & Mean CES-D Score \\
\hline $\begin{array}{l}\text { Physical Disability } \\
\text { (PD)(Main Effect) }\end{array}$ & $\mathrm{F}(2,3895)=62.30$ & $\mathrm{p}<0.001$ & $\begin{array}{l}0 \text { limitations }=2.68 \\
1 \text { limitation }=3.80 \\
2+\text { limitations }=5.11\end{array}$ \\
\hline $\begin{array}{l}\text { Overall Medical Burden } \\
(\text { OMB) (Main Effect) }\end{array}$ & $\mathrm{F}(3,3895)=8.31$ & $\mathrm{p}<0.001$ & $\begin{array}{l}0 \text { conditions }=3.19 \\
1 \text { condition }=3.66 \\
2 \text { conditions }=4.06 \\
3+\text { conditions }=4.55\end{array}$ \\
\hline $\begin{array}{l}\text { PD X OMB } \\
(2-\text { Way Interaction) }\end{array}$ & $\mathrm{F}(6,3895)=1.91$ & $\mathrm{p}=0.08$ & $\mathrm{p}=0.30$ \\
\hline $\begin{array}{l}\text { PD X County Code } \\
(2-\text { Way Interaction) }\end{array}$ & $\mathrm{F}(6,3895)=1.21$ & $\mathrm{p}=0.49$ & \\
\hline $\begin{array}{l}\text { PD X OMB X County } \\
\text { Code (3-Way Interaction) }\end{array}$ & $\mathrm{F}(18,3895)=0.97$ & & \\
\hline
\end{tabular}


Table 18

Physical disability (PD), healthcare utilization, county code, and depression

\begin{tabular}{|c|c|c|c|}
\hline Health Disparity & Test of Interaction & Significance & Mean CES-D Score \\
\hline $\begin{array}{l}\text { Physical Disability (PD) } \\
\text { (Main Effect) }\end{array}$ & $F(2,3744)=50.69$ & $\mathrm{p}<0.001$ & $\begin{array}{l}0 \text { limitations }=2.77 \\
1 \text { limitation }=4.07 \\
2+\text { limitations }=5.16\end{array}$ \\
\hline $\begin{array}{l}\text { Healthcare Utilization } \\
\text { (HCU) (Main Effect) }\end{array}$ & $F(4,3744)=9.44$ & $\mathrm{p}<0.001$ & $\begin{array}{l}0 \text { visits }=3.51 \\
1-3 \text { visits }=3.29 \\
4-6 \text { visits }=3.79 \\
7-12 \text { visits }=4.28 \\
>12 \text { visits }=5.11\end{array}$ \\
\hline $\begin{array}{l}\text { PD X HCU } \\
\text { (2-Way Interaction) }\end{array}$ & $F(8,3744)=0.64$ & $p=0.75$ & \\
\hline $\begin{array}{l}\text { PD X County Code } \\
\text { (2-Way Interaction) }\end{array}$ & $\mathrm{F}(6,3744)=1.10$ & $p=0.36$ & \\
\hline $\begin{array}{l}\text { PD X HCU X County } \\
\text { Code ( } 3-\text { Way Interaction) }\end{array}$ & $F(24,3744)=1.02$ & $\mathrm{p}=0.43$ & \\
\hline
\end{tabular}


Table 19

Differences in self-efficacy and negative attitudes towards doctors by county code

\begin{tabular}{|c|c|c|c|c|c|}
\hline $\begin{array}{l}\text { Culture } \\
\text { Variable }\end{array}$ & Test & Significance & \begin{tabular}{|l|} 
Post Hoc \\
Comparisons
\end{tabular} & $\begin{array}{l}\text { County Code } \\
\text { (n) }\end{array}$ & $\begin{array}{l}\text { Mean } \\
\text { Score }\end{array}$ \\
\hline $\begin{array}{l}\text { Negative } \\
\text { Attitudes } \\
(\mathrm{n}=1,471)\end{array}$ & $\begin{array}{l}\mathrm{F}(2,1468)= \\
31.58\end{array}$ & $\mathrm{p}<0.001$ & $\begin{array}{l}2 \text { vs. } 8 p< \\
0.001 \\
6 \text { vs. } 8 \text { p }< \\
0.001 \\
2 \text { vs. } 6 \mathrm{p}= \\
0.02\end{array}$ & $\begin{array}{l}2(\mathrm{n}=918) \\
6(\mathrm{n}=396) \\
8(\mathrm{n}=156)\end{array}$ & $\begin{array}{l}21.17 \\
21.63 \\
23.08\end{array}$ \\
\hline $\begin{array}{l}\text { Self- } \\
\text { Efficacy } \\
(\mathrm{n}=2,395)\end{array}$ & $\begin{array}{l}\mathrm{F}(2,2392)= \\
4.074\end{array}$ & $p=0.017$ & $\begin{array}{l}\text { Codes } 2 \text { vs. } 8 \\
p=0.03\end{array}$ & $\begin{array}{l}2(\mathrm{n}=1,441) \\
6(\mathrm{n}=705) \\
8(\mathrm{n}=249)\end{array}$ & $\begin{array}{l}4.51 \\
4.36 \\
4.18 \\
\end{array}$ \\
\hline
\end{tabular}

Wave 2: 3-levels of county code 
Table 20

Self efficacy and overall medical burden

\begin{tabular}{|l|l|l|l|}
\hline $\begin{array}{l}\text { Overall Medical } \\
\text { Burden }\end{array}$ & $\begin{array}{l}\text { Low Self-efficacy } \\
(\boldsymbol{\%})\end{array}$ & $\begin{array}{l}\text { Moderate } \\
\text { Self-efficacy }(\boldsymbol{\%})\end{array}$ & $\begin{array}{l}\text { High Self-efficacy } \\
(\boldsymbol{\%})\end{array}$ \\
\hline 0 conditions & 21.2 & 23.3 & 29.6 \\
\hline 1 condition & 34.2 & 41.4 & 38.9 \\
\hline 2 conditions & 28.4 & 21.7 & 21.7 \\
\hline $3+$ conditions & 16.2 & 13.5 & 9.8 \\
\hline
\end{tabular}


Table 21

Rates of incident depression (CES-D $\geq 9$ ) at follow-up by county code

\begin{tabular}{|l|l|l|l|l|}
\hline In-Person Wave & Test & Significance & County Code & $\begin{array}{l}\text { \% Incident } \\
\text { depression }\end{array}$ \\
\hline Wave $1(\mathrm{n}=3,996)$ & -- & -- & 2 & 10.1 \\
& & & 6 & 9.1 \\
& & & 7 & 8.4 \\
& & & 9 & 8.9 \\
\hline Wave 2* $(\mathrm{n}=2,407)$ & $\chi^{2}(3)=1.94$ & $\mathrm{p}=0.58$ & 2 & 4.0 \\
& & & 6 & 4.9 \\
& & & 7 & 2.9 \\
\hline Wave $3^{*}(\mathrm{n}=1,787)$ & $\chi^{2}(3)=2.12$ & $\mathrm{p}=0.55$ & 2 & 4.5 \\
& & & 6 & 5.1 \\
& & & 7 & 6.5 \\
& & & 9 & 4.2 \\
\hline Wave $4^{*}(\mathrm{n}=1,168)$ & $\chi^{2}(3)=7.69$ & $\mathrm{p}=0.05$ & 2 & 6.6 \\
& & & 6 & 5.0 \\
& & & 7 & 5.2 \\
& & & 9 & 10.4 \\
\hline
\end{tabular}

* In participants without significant depressive symptomotology at baseline (CES-D $\geq 9$ ) 


\section{Appendix B: List of figures}

Figure 1

CES-D score frequency (possible score 0-20, sample range 0-17)

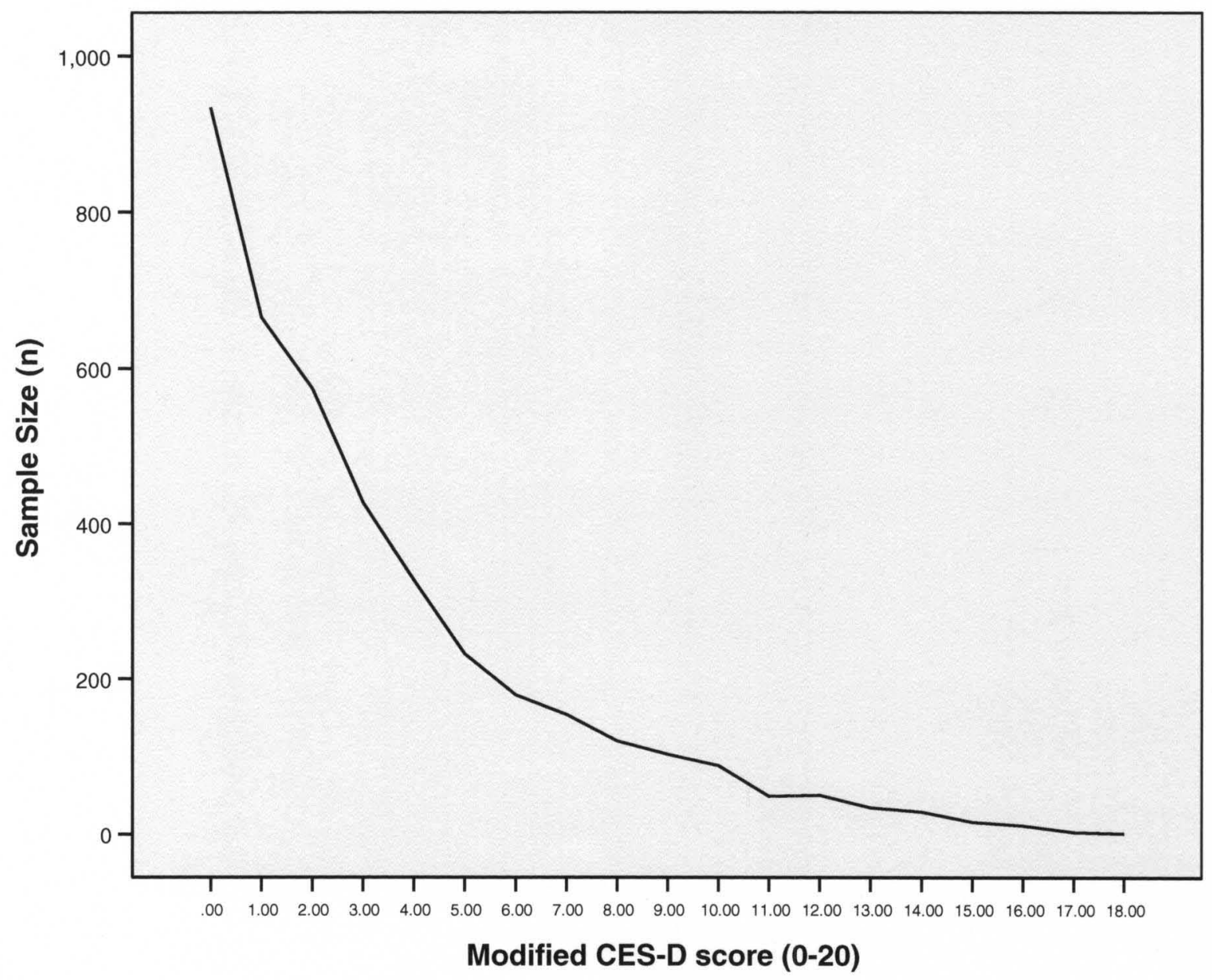


Figure 2

Mean depression score for levels of overall medical burden by county code

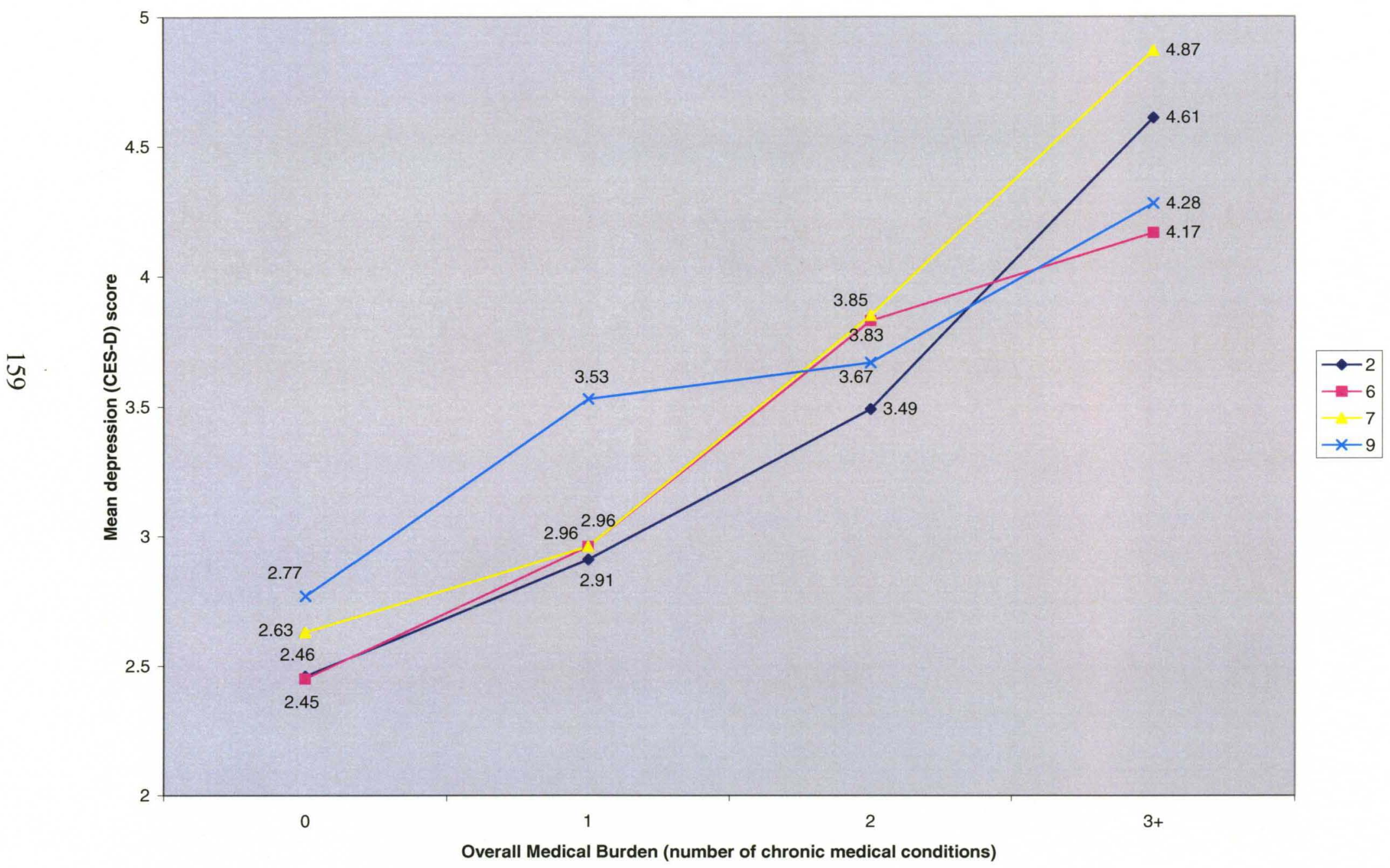


Figure 3

Mean depression score for levels of HCU by county code

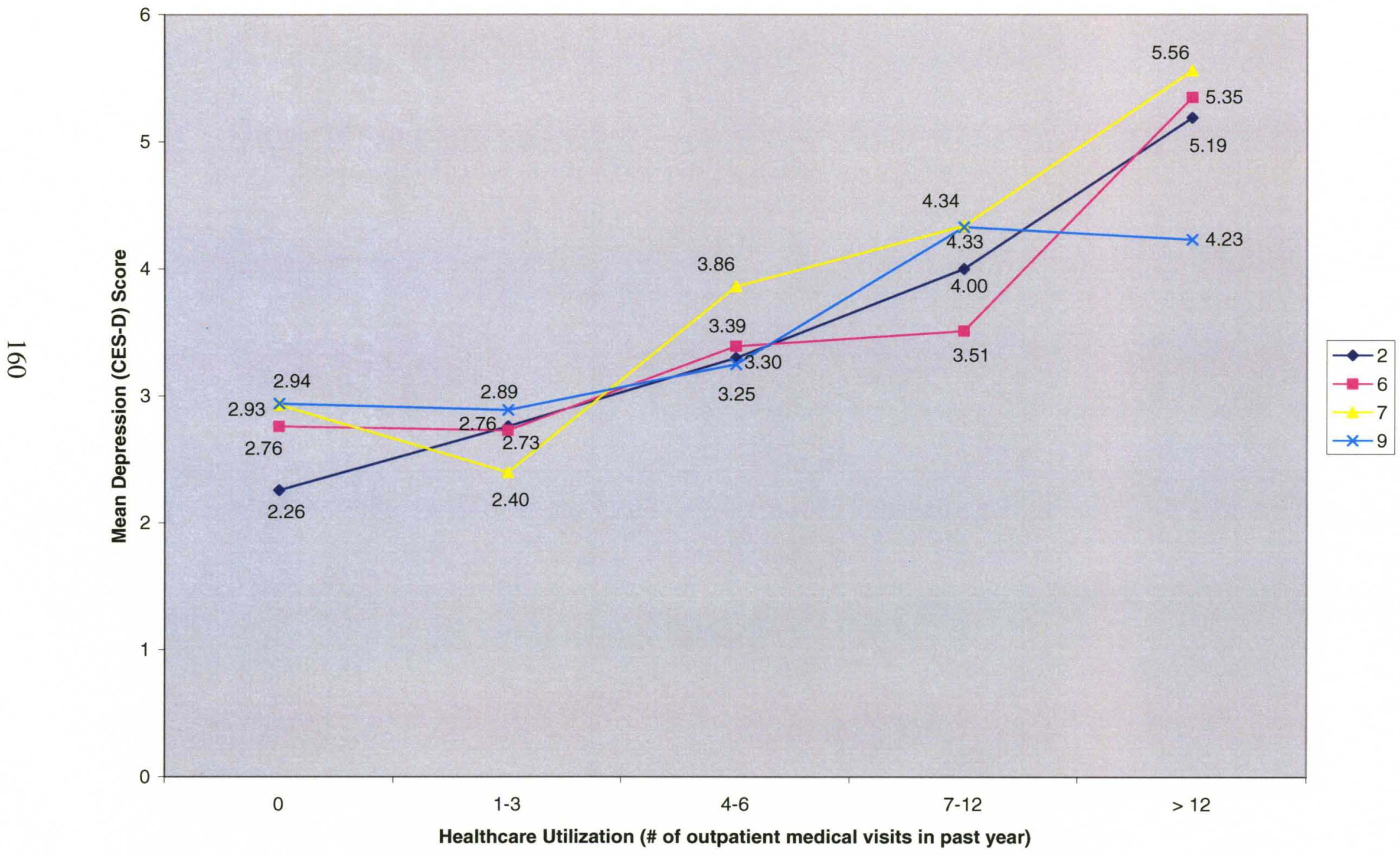


Figure 4

Mean depression score for levels of NHBs by county code

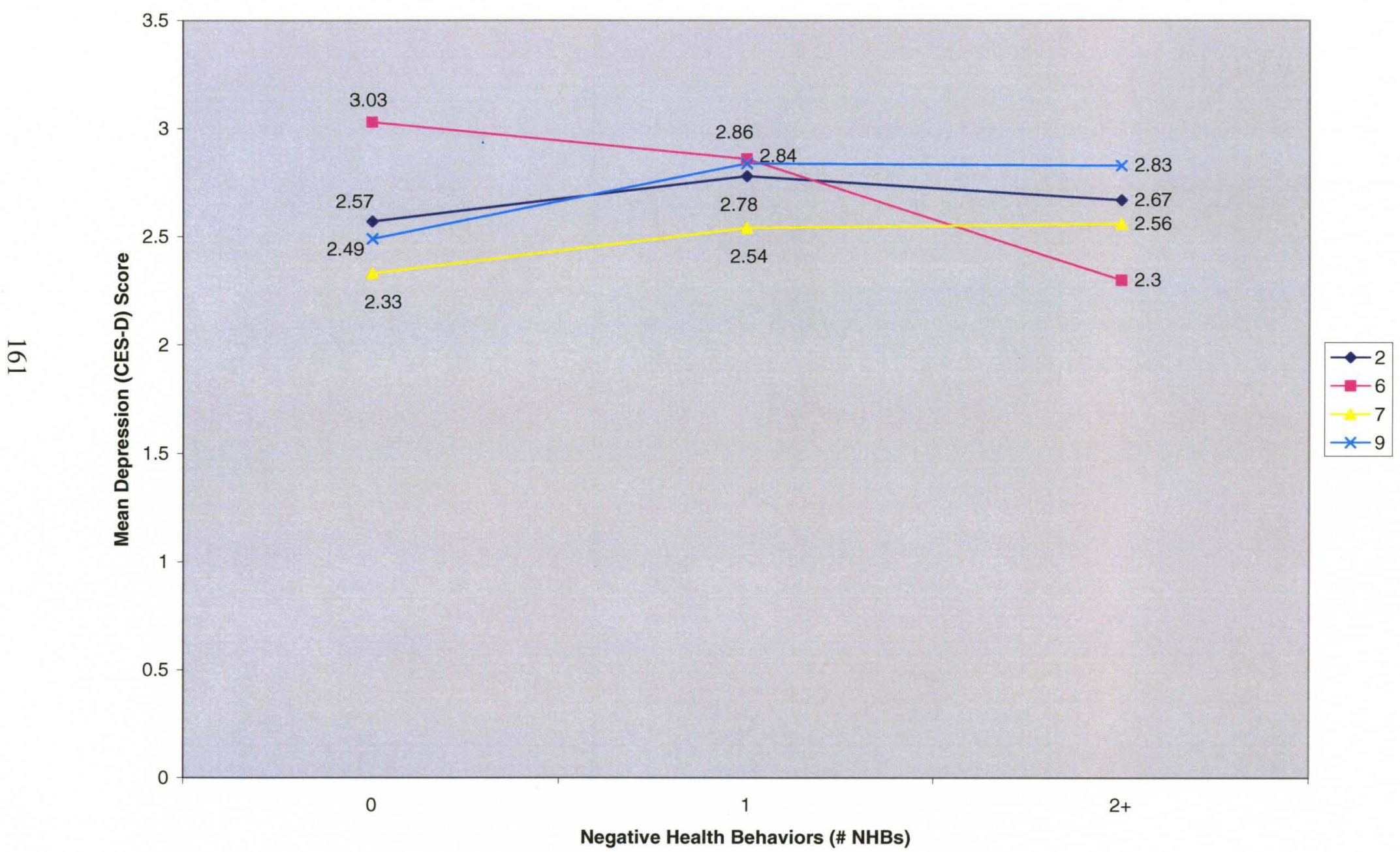


Figure 5

Mean depression score for levels of CVR by level of rurality

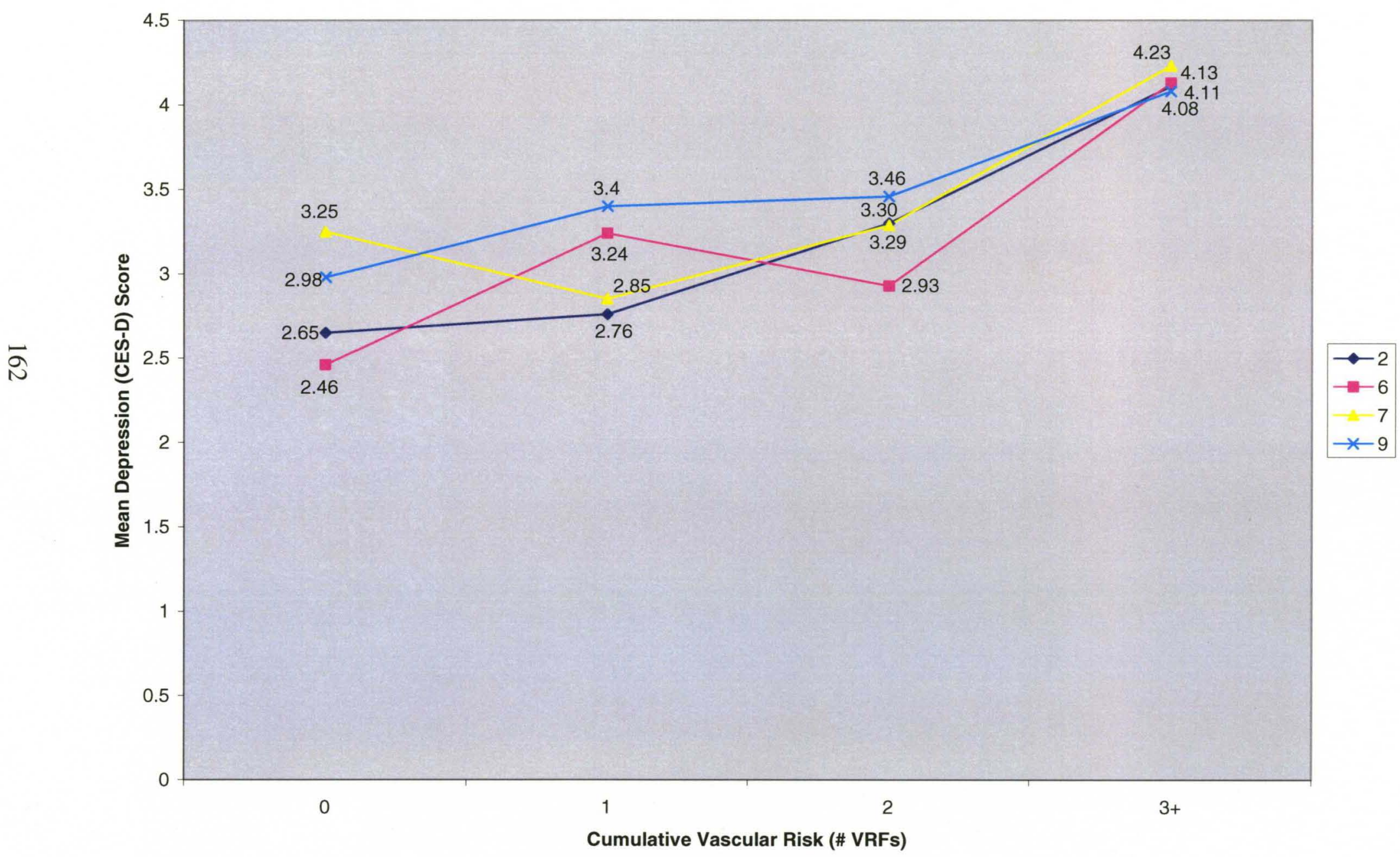


Figure 6

Percent of Blacks and Non-Hispanic Whites with prevalent depression (CES-D $\geq 9$ ) by county code

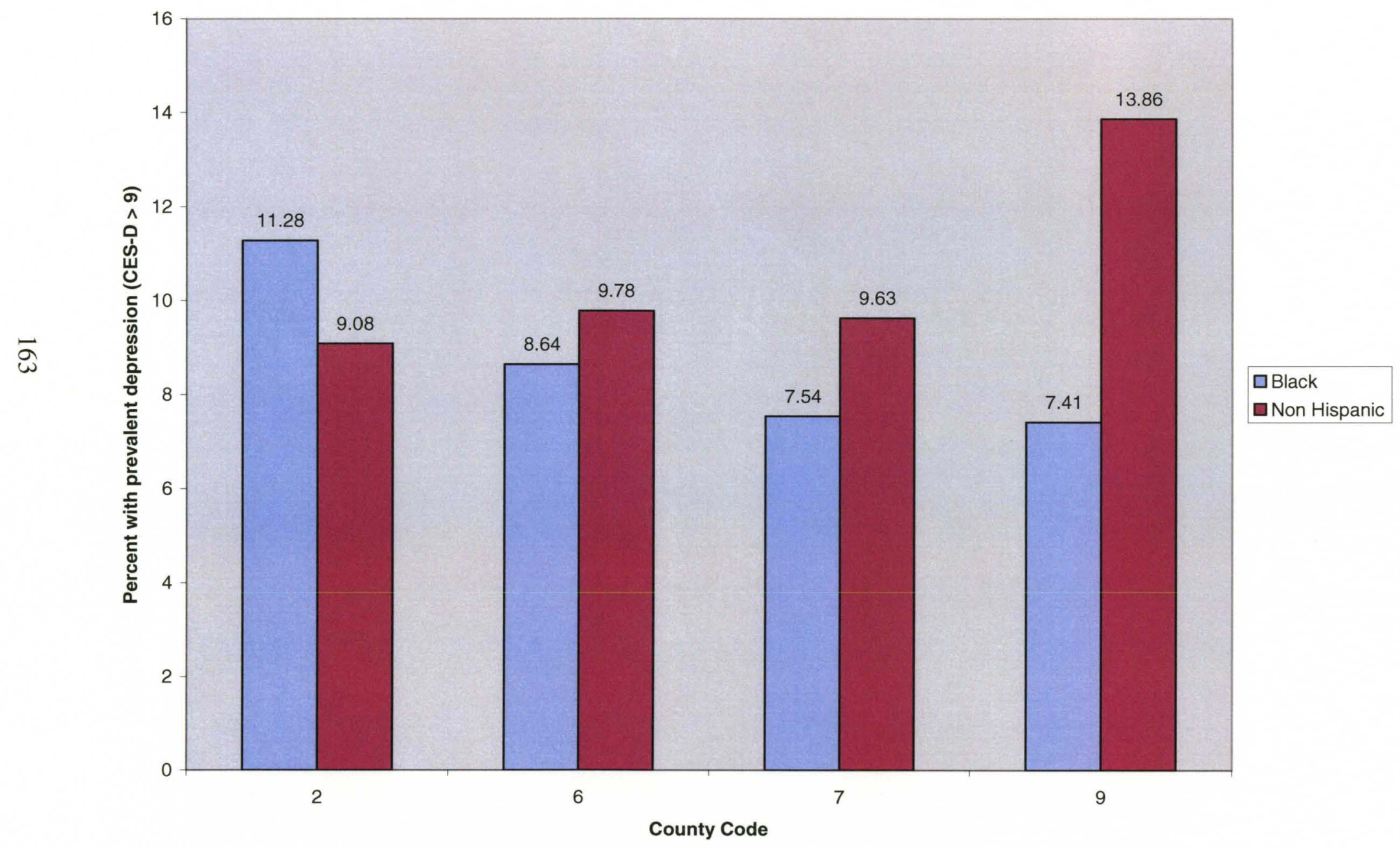




\section{Appendix C: Attitudes towards Doctors Items}

1) Doctors always do their best to keep patients as old as I am from worrying.

2) Doctors always treat their patients my age with respect.

3) Sometimes doctors make patients my age feel foolish.

4) When treating people about my age doctors always avoid unnecessary patient expenses.

5) Doctors often cause patients my age to worry a lot, because they don't explain things well.

6) When treating people about my age, doctors respect their patients' feelings.

7) Doctors never recommend an operation for people my age, unless there is no other way to solve the problem.

8) Doctors don't pay enough attention to the health problems that people my age have.

9) Most of these younger doctors really understand how people my age feel.

10) Sometimes doctors think that just getting old is a disease that can't be cured. 


\section{Appendix D: Adapted Pearlin Mastery Scale: Self-efficacy items}

1) I have little control over the things that happen to me.

2) What happens to me in the future mostly depends on me.

3) There is really no way I can solve some of the problems I have.

4) There is little I can do to change many of the important things in my life.

5) I can do just about anything I really set my mind to do.

6) I often feel helpless in dealing with the problems in life.

7) Sometimes I feel that I'm being pushed around in life. 
Appendix E: Associations between main HD, culture, and potential moderator variables

\begin{tabular}{|c|c|c|c|c|c|c|c|c|}
\hline & OMB & CVR & $\mathrm{HCU}$ & $\mathrm{NHBs}^{\mathrm{a}}$ & $\mathbf{N A s} \mathbf{s}^{\mathbf{a}}$ & $\begin{array}{l}\text { Self- } \\
\text { efficacy }^{a}\end{array}$ & $\begin{array}{l}\text { Perceived } \\
\text { Social } \\
\text { Support }\end{array}$ & $\begin{array}{l}\text { Physical } \\
\text { Disability }\end{array}$ \\
\hline OMB & & & & & & & & \\
\hline CVR & $\begin{array}{l}\chi^{2}(9)= \\
4223.56^{* *}\end{array}$ & & & & & & & \\
\hline HCU & $\begin{array}{l}\chi^{2}(12)= \\
398.28 * *\end{array}$ & $\begin{array}{l}\chi^{2}(12)= \\
202.80 * *\end{array}$ & & & & & & \\
\hline NHBs & $\begin{array}{l}\chi^{2}(6)= \\
4.51\end{array}$ & $\begin{array}{l}\chi^{2}(6)= \\
415.27 * *\end{array}$ & $\begin{array}{l}\chi^{2}(8)= \\
23.42 *\end{array}$ & & & & & \\
\hline NAs & $\begin{array}{l}\chi^{2}(6)= \\
9.66\end{array}$ & $\begin{array}{l}\chi^{2}(6)= \\
6.40\end{array}$ & $\begin{array}{l}\chi^{2}(8)= \\
8.56\end{array}$ & $\begin{array}{l}\chi^{2}(4)= \\
8.61\end{array}$ & & & & \\
\hline $\begin{array}{l}\text { Self- } \\
\text { efficacy }\end{array}$ & $\begin{array}{l}\chi^{2}(6)= \\
29.04 * *\end{array}$ & $\begin{array}{l}\chi^{2}(6)= \\
6.98\end{array}$ & $\begin{array}{l}\chi^{2}(8)= \\
61.01 * *\end{array}$ & $\begin{array}{l}\chi^{2}(4)= \\
5.37\end{array}$ & $\begin{array}{l}\chi^{2}(4)= \\
90.69 * *\end{array}$ & & & \\
\hline $\begin{array}{l}\text { Perceived } \\
\text { Social } \\
\text { Support }\end{array}$ & $\begin{array}{l}\chi^{2}(9)= \\
20.75 *\end{array}$ & $\begin{array}{l}\chi^{2}(9)= \\
19.60\end{array}$ & $\begin{array}{l}\chi^{2}(12)= \\
20.26\end{array}$ & $\begin{array}{l}\chi^{2}(6)= \\
14.09\end{array}$ & $\begin{array}{l}\chi^{2}(6)= \\
31.93 * *\end{array}$ & $\begin{array}{l}\chi^{2}(6)= \\
90.55 * *\end{array}$ & & \\
\hline $\begin{array}{l}\text { Physical } \\
\text { Disability }\end{array}$ & $\begin{array}{l}\chi^{2}(6)= \\
211.09 * *\end{array}$ & $\begin{array}{l}\chi^{2}(6)= \\
103.39 * *\end{array}$ & $\begin{array}{l}\chi^{2}(8)= \\
212.42 * *\end{array}$ & $\begin{array}{l}\chi^{2}(4)= \\
4.07\end{array}$ & $\begin{array}{l}\chi^{2}(4)= \\
27.54 * *\end{array}$ & $\begin{array}{l}\chi^{2}(4)= \\
293.23 * *\end{array}$ & $\begin{array}{l}\chi^{2}(6)= \\
27.41 * *\end{array}$ & \\
\hline
\end{tabular}


Appendix F: Frequency counts for main study variables for the sample as a whole $(\mathbf{n}=3,996)$

Overall Medical Burden (\# of chronic medical conditions)

\begin{tabular}{|l|l|l|l|}
\hline & Frequency & Percent & Cumulative Percent \\
\hline 0 & 965 & 24.1 & 24.3 \\
\hline 1 & 1485 & 37.2 & 61.7 \\
\hline 2 & 982 & 24.6 & 86.4 \\
\hline $3+$ & 540 & 13.5 & 100.0 \\
\hline Total & 3972 & 99.4 & \\
\hline
\end{tabular}

Cumulative Vascular Risk (CVR; \# of vascular risk factors)

\begin{tabular}{|l|l|l|l|}
\hline & Frequency & Percent & Cumulative Percent \\
\hline 0 & 576 & 14.4 & 14.5 \\
\hline 1 & 1408 & 35.2 & 50.1 \\
\hline 2 & 1201 & 30.1 & 80.4 \\
\hline $3+$ & 778 & 19.5 & 100.0 \\
\hline Total & 3963 & 99.2 & \\
\hline
\end{tabular}

HCU (\# of outpatient medical visits in the past year)

\begin{tabular}{|l|l|l|l|}
\hline & Frequency & Percent & Cumulative Percent \\
\hline 0 & 740 & 18.5 & 19.3 \\
\hline $1-3$ & 1382 & 34.6 & 55.4 \\
\hline $4-6$ & 913 & 22.8 & 79.2 \\
\hline $7-12$ & 548 & 13.7 & 93.6 \\
\hline$>12$ & 247 & 6.2 & 100.0 \\
\hline Total & 3830 & 95.8 & \\
\hline
\end{tabular}

Negative Health Behaviors (NHBs; \# of NHBs)

\begin{tabular}{|l|l|l|l|}
\hline & Frequency & Percent & Cumulative Percent \\
\hline 0 & 531 & 13.3 & 18.9 \\
\hline 1 & 1492 & 37.3 & 72.0 \\
\hline $2+$ & 788 & 19.7 & 100.0 \\
\hline Total & 2811 & 70.3 & \\
\hline
\end{tabular}

Heart Attack

\begin{tabular}{|l|l|l|l|}
\hline & Frequency & Percent & Cumulative Percent \\
\hline No & 3385 & 84.7 & 84.9 \\
\hline Yes & 602 & 15.1 & 100.0 \\
\hline Total & 3987 & 99.8 & \\
\hline
\end{tabular}


Obesity

\begin{tabular}{|l|l|l|l|}
\hline & Frequency & Percent & Cumulative Percent \\
\hline No & 3352 & 83.9 & 83.9 \\
\hline Yes & 644 & 16.1 & 100.0 \\
\hline Total & 3996 & 100.0 & \\
\hline
\end{tabular}

Hypertension

\begin{tabular}{|l|l|l|l|}
\hline & Frequency & Percent & Cumulative Percent \\
\hline No & 1693 & 42.4 & 42.4 \\
\hline Yes & 2299 & 57.5 & 100.0 \\
\hline Total & 3992 & 99.9 & \\
\hline
\end{tabular}

Diabetes

\begin{tabular}{|l|l|l|l|}
\hline & Frequency & Percent & Cumulative Percent \\
\hline No & 3185 & 79.7 & 79.9 \\
\hline Yes & 803 & 20.1 & 100.0 \\
\hline Total & 3988 & 99.8 & \\
\hline
\end{tabular}

Broken Hip

\begin{tabular}{|l|l|l|l|}
\hline & Frequency & Percent & Cumulative Percent \\
\hline No & 3854 & 96.4 & 96.5 \\
\hline Yes & 141 & 3.5 & 100.0 \\
\hline Total & 3995 & 100.0 & \\
\hline
\end{tabular}

Stroke

\begin{tabular}{|l|l|l|l|}
\hline & Frequency & Percent & Cumulative Percent \\
\hline No & 3682 & 92.1 & 92.2 \\
\hline Yes & 313 & 7.8 & 100.0 \\
\hline Total & 3995 & 100.0 & \\
\hline
\end{tabular}

Cancer

\begin{tabular}{|l|l|l|l|}
\hline & Frequency & Percent & Cumulative Percent \\
\hline No & 3534 & 88.4 & 88.5 \\
\hline Yes & 460 & 11.5 & 100.0 \\
\hline Total & 3994 & 99.9 & \\
\hline
\end{tabular}

Alcohol Risk

\begin{tabular}{|l|l|l|l|}
\hline & Frequency & Percent & Cumulative Percent \\
\hline No & 2907 & 72.7 & 98.8 \\
\hline Yes & 34 & 0.9 & 100.0 \\
\hline Total & 2941 & 73.6 & \\
\hline
\end{tabular}


Exercise Risk

\begin{tabular}{|l|l|l|l|}
\hline & Frequency & Percent & Cumulative Percent \\
\hline No & 971 & 24.3 & 32.6 \\
\hline Yes & 2008 & 50.3 & 100.0 \\
\hline Total & 2979 & 74.5 & \\
\hline
\end{tabular}

Smoke Risk

\begin{tabular}{|l|l|l|l|}
\hline & Frequency & Percent & Cumulative Percent \\
\hline No & 2204 & $\mathbf{5 5 . 2}$ & 67.0 \\
\hline Yes & 1086 & 27.2 & 100.0 \\
\hline Total & 3290 & 82.3 & \\
\hline
\end{tabular}

Physical Disability (\# of impairments in ADLs)

\begin{tabular}{|l|l|l|l|}
\hline & Frequency & Percent & Cumulative Percent \\
\hline 0 & 2197 & 55.0 & 55.3 \\
\hline 1 & 1251 & 31.3 & 86.8 \\
\hline $2+$ & 524 & 13.1 & 100.0 \\
\hline Total & 3972 & 99.4 & \\
\hline
\end{tabular}

Perceived Social Support

\begin{tabular}{|l|l|l|l|}
\hline & Frequency & Percent & Cumulative Percent \\
\hline Low & 173 & 4.3 & 4.4 \\
\hline Moderate & 455 & 11.4 & 15.8 \\
\hline High & 619 & 15.5 & 31.4 \\
\hline Very High & 2728 & 68.3 & 100.0 \\
\hline Total & 3975 & 99.5 & \\
\hline
\end{tabular}

Self-Efficacy

\begin{tabular}{|l|l|l|l|}
\hline & Frequency & Percent & Cumulative Percent \\
\hline Low & 770 & 19.3 & 32.2 \\
\hline Moderate & 808 & 20.2 & 65.9 \\
\hline High & 817 & 20.4 & 100.0 \\
\hline Total & 2395 & 59.9 & \\
\hline
\end{tabular}

Negative Attitudes towards Medical Doctors (NAs)

\begin{tabular}{|l|l|l|l|}
\hline & Frequency & Percent & Cumulative Percent \\
\hline Low & 522 & 13.1 & 35.5 \\
\hline Moderate & 500 & 12.5 & 69.5 \\
\hline High & 449 & 11.2 & 100.0 \\
\hline Total & 1471 & 36.8 & \\
\hline
\end{tabular}




\section{CURRICULUM VITAE}

\section{Jennifer A. Zimmerman, M.A.}

\section{EDUCATION}

UNIVERSITY OF LOUISVILLE, Louisville, Kentucky

August 2006-Present

Clinical Psychology Ph.D. Program

Expected Date of Completion: May 2011

Current GPA: 4.0

Faculty Mentor: Benjamin Mast, Ph.D.

Dissertation: Health Disparities and Depression in Rural and Urban Older Adults Successfully defended on July 27, 2010

Chair: Benjamin Mast, Ph.D

UNIVERSITY OF LOUISVILLE, Louisville, Kentucky

Masters of Arts, Clinical Psychology: December 2008

Overall GPA: 4.0

Faculty Mentor: Benjamin Mast, Ph.D.

MARIST COLLEGE, Poughkeepsie, New York

Bachelor of Arts, Psychology: May 2004

Minor: Biology

Overall GPA: 3.93 Major GPA: 3.98

HONORS:

Graduated Summa Cum Laude

Received the Psychology Undergraduate Baccalaureate Award

Dean's List: All semesters

Deans' Circle

Psi Chi Honor Society in Psychology

Alpha Chi Honor Society

Who's Who Among Students in American Universities and Colleges 


\section{CLINICAL EXPERIENCE}

Clinical Internship

Pittsburgh VA Healthcare System, Pittsburgh, PA, to be completed: August, 2010 August 2011

\section{Graduate Student Therapist}

Geropsychology Assessment and Intervention Team, University of Louisville, Louisville, KY, August 2009 - Present

Supervisor: Benjamin Mast, Ph.D.

- Conduct neuropsychological assessments on outpatient older adults in mental health and primary care settings. Assessments involve chart review, integrative report writing, providing feedback to patients, caregivers, patient's geriatrician and treatment team, and working within an interdisciplinary team in the geriatric outpatient clinic setting.

- Conduct psychotherapy with older adult clients and dementia caregivers.

\section{Graduate Student Therapist}

Integrative Psychotherapy, University of Louisville, Louisville, KY, August 2009 Present

Supervisor: Jay Irby, Ph.D.

- Conduct individual therapy with adult clients in the Clinical Psychology University Clinic

\section{Graduate Student Therapist}

Central State Psychiatric Hospital, Louisville, KY, July 2008 - June 2009

Supervisor: J. Wayne Putnam, PsyD

- Conduct individual therapy, group therapy, and psychodiagnostic, neuropsychological, and malingering assessments with patients on an acute unit of an inpatient psychiatric hospital

- All patients presented with severe mental illness

- Participate in treatment planning and work within an interdisciplinary team

\section{Graduate Student Therapist}

Interpersonal Therapy Team, Psychological Services Center, University of Louisville, University of Louisville, Louisville VA, August 2008-Ausgust 2009 Supervisor: Stanley Murrell, Ph.D.

- Conduct individual time-limited interpersonal psychotherapy with individuals experiencing interpersonal difficulties

\section{Graduate Student Therapist/Assessment Practicum}

Geriatric Clinic, University of Louisville, Louisville, KY, May 2007 - May 2008 Supervisor: Benjamin Mast, Ph.D.

- Conduct neuropsychiatric assessments to aid in diagnosis and treatment management

- Write integrative reports for geriatrician and treatment team, patient, and caregivers

- Provide feedback sessions for patient and caregivers 


\section{Graduate Student Therapist}

Veterans Affairs Home-Based Primary Care Psychology Team, University of Louisville, Louisville VA, August 2006 - August 2008

Supervisor: Barbara Stetson, Ph.D.

- Conduct individual psychotherapy with older adult veterans in their home environment

\section{Graduate Student Therapist}

Psychological Assessments, Psychological Services Center, university of Louisville, March 2007-present

Supervisor: Paul Bock, Ph.D.; Bernadette Walter, Ph.D.; David Winsch, Ph.D.

- Conduct semi-structured clinical interviews

- Conduct ADHD, LD, MR, full diagnostic and intellectual functioning assessments.

- Administration of intelligence, achievement, diagnostic, and personality tests.

- Conduct intellectual assessments on primary-school aged children for placement in Louisville's public school advanced placement program.

\section{Graduate Student Therapist/Assessment Practicum}

Wedgewood Healthcare Center (Nursing Home/Rehabilitation), Clarkesville, Indiana, May 2008 - August 2008

Supervisor: Suzanne Meeks, Ph.D.

- Conduct neuropsychological and psychological assessment of nursing home residents

\section{RESEARCH EXPERIENCE}

\section{Health Disparities and Depression in Rural and Urban Older Adults}

Dissertation Study

University of Louisville, April, 2009 - Present

Supervisor: Benjamin Mast, Ph.D.

- Project Coordinator (literature review, development of study hypotheses and methods, database management, data analysis, writing and proposal of final study document)

- Investigates the prevalence of health disparities in rural and urban OAs and examine the relationship between health disparities and depression risk and severity based on county-determined level of rurality

- Utilizes a baseline sample of 3,996 older adults from the Duke Established of Populations for Epidemiologic Studies of the Elderly (EPESE): Piedmont Health Survey of the Elderly

\section{Meta-Analysis of the Vascular Depression Hypothesis}

University of Louisville, 2008 - Present

Supervisor: Benjamin Mast, Ph.D.

- Project Coordinator: overseeing project and delegation of tasks to other graduate and undergraduate students

- Collecting relevant articles through thorough literature review 
- Coding articles/recording relevant data and study information

- Calculation of appropriate effect size statistics

- Database creation, entry, and maintenance

- Statistical analysis

- Primary author on current manuscript (currently in the writing process)

Investigating Vascular Depression in a Hispanic-American Sample University of Louisville, $2007-2008$

Supervisor: Benjamin Mast, Ph.D.

- Utilized a secondary dataset: the Hispanic Established Population for the Epidemiologic Study of the Elderly (EPESE) to investigate the vascular depression hypothesis in a sample consisting of 2,489 community-dwelling Mexican American elders

Research Assistant, University of Louisville, 2006-2007

Early Detection Trial of Sub-clinical Cognitive Impairment

Principle Investigator: Toni Miles, M.D., Ph.D.

- Recruitment of participants

- Neuropsychological assessment

- Participated in research meetings discussing participants and deciding on referrals if appropriate (based on interview, test results, and MRI results)

Senior Research Assistant, Cornell Institute of Geriatric Psychiatry, part of Weill Medical College of Cornell University, July 2004-July 2006

\section{Studies:}

Aging white matter changes, executive dysfunction and depression

Escitalopram vs. placebo for the prevention of relapse and recurrence of geriatric depression

Psychotherapy in geriatric depression with executive dysfunction

Treatment effectiveness in depressed patients with COPD

Principle Investigator: George Alexopoulos, M.D.

- Assist Principle Investigator and associated investigators with multiple NIMH funded research studies in treatment of geriatric mood disorders

- Coordinator of Control Subjects: Monitor and track subjects throughout course of study; Supervise research assistants in research data collection

- Responsible for tracking, reviewing, and submitting adverse events

- Screen, evaluate, and recruit potential study subjects

- Participate in community outreach activities/events

- Conduct structured clinical interviews, (SCID)

- Administer a large research battery of neuropsychological measures consisting of tests of cognitive functioning, depression severity, physical functioning

\section{OTHER RESEARCH EXPERIENCE}

Principle Researcher, Marist College, Poughkeepsie, NY, 2003-2004 
Marist College Undergraduate Research Partnership, Stipend Awarded

Influence of Endurance Performance on Salivary Serotonin Concentration in Female Athletes.

- Applied immunohistochemistry by using the ELISA test to determine the concentration of serotonin in the saliva of college-aged female cross country runners after runs of different distances

Research Assistant, Marist College, Poughkeepsie, NY, 2003-2004

Social Norms Marketing Research Project. Funded by the Education Development Center, Inc.

- SNMPR is a national, multi-site study to test the effectiveness of a social norms marketing campaign, Just the Facts (JTF), in reducing high-risk drinking among college students.

- Involved in data collection and analysis; presented "Just the Facts" presentation multiple times to different groups of Marist College students; helped organize and run focus groups on advertisement strategies

- Principle Researcher: Steve Sansola

Co-Researcher, Marist College, Poughkeepsie, NY, Summer 2003

Bioaccumulation of Cadmium in Blue Gills (Lepomis marcrochirus).

- Designed behavioral study, trained research team in and participated in dissection of fish, trained researchers how to use histology equipment, and participated in histological analysis.

Co-Researcher, Marist College, Poughkeepsie, NY, 2002-2003

Histological study of the effects of platinum, palladium, and rhodium on rats (Mus musculus).

- Tissue embedding in paraffin wax, sectioning, Hematoxylin/eosin staining, and analysis of pathological damage to tissues.

\section{PUBLICATIONS}

Zimmerman, J. A., Fiske, A., Scogin, F. (In review). Mentoring in clinical geropsychology: Across the stages of professional development. Invited Introduction Article. Journal of Educational Gerontology.

Zimmerman, J. A., Mast, B. T., Miles, T., \& Markides, K. S. (2009). Vascular risk and depression in the Hispanic Established Population for the Epidemiologic Study of the Elderly (EPESE). International Journal of Geriatric Psychiatry, 24: 409-416.

Mast, B.T., Zimmerman, J., \& Rowe, S.V. (2009). What do we know about the aging brain? Implications for learning in late life (p. 695-731). In M.C. Smith, \& T.G. Reio, Jr., (Eds.) Handbook of Research on Adult Development and Learning. New York, NY:

Routledge. 


\section{PUBLISHED ABSTRACTS}

Zimmerman, J. A., Fiske, A. (November, 2009). Mentoring Across the Geropsychology Career: Perspectives from the Experts. Proceedings of the $62^{\text {nd }}$ Annual Scientific meeting of the Gerontological Society of America. Atlanta, GA.

Zimmerman, J., Holley, C.K., Rowe, S.V., Healy, P.J., \& Mast, B.T. (November 2008). Meta-analysis of the vascular depression hypothesis. Proceedings from the 61st Annual Scientific meeting of the Gerontological Society of America. National Harbor, MD.

Zimmerman, J. A., Mast, B. T., Miles, T., \& Markides, K. S. (November, 2007). Vascular Risk and Depression in the Hispanic Established Population for the Epidemiologic Study of the Elderly (EPESE). Proceedings from the $60^{\text {th }}$ Annual meeting of the Gerontological Society of America. San Francisco, CA.

Zimmerman, J., Gagnon, Z. (2004). "Endurance exercise induced changes in salivary serotonin concentrations." Proceedings of the $58^{\text {th }}$ Annual Eastern Colleges Science Conference, Manhattan College, New York

Reeves, B., Zimmerman, J., Baker, C., Porzio, V., Gagnon, Z., Connetta, J., Newkirk, C. (2003) "Histopathological Study of Platinum Group Metal Exposure to Rats."

Proceedings of the $57^{\text {th }}$ Annual Eastern Colleges Science Conference. Ithaca, New York

Reeves, B., Zimmerman, J., Baker, C., Porzio, V., Gagnon, Z., Connetta, J., Newkirk, C. (2003) "Pathological Effects of Platinum, Palladium, and Rhodium in Rattus norvegicus Tissues." American Chemical Society Mid-Hudson Chapter Undergraduate Research Symposium. Poughkeepsie, New York

\section{PRESENTATIONS AND AWARDS}

Zimmerman, J. A., \& Mast, B. Rural Culture and Healthcare Utilization. (November, 2010). Poster Presentation to be presented at the $63^{\text {rd }}$ Annual Scientific meeting of the Gerontological Society of America. New Orleans, IL.

Zimmerman, J. A., \& Fiske, A. (November, 2009). Symposium: Mentoring Across the Geropsychology Career: Perspectives from the Experts. Presented at the $62^{\text {nd }}$ Annual Scientific meeting of the Gerontological Society of America. Atlanta, GA

Zimmerman, J., Holley, C.K., Rowe, S.V., Healy, P.J., \& Mast, B.T. (November, 2008). Meta-analysis of the vascular depression hypothesis. Poster presented at the 61st Annual Scientific meeting of the Gerontological Society of America. National Harbor, MD

Zimmerman, J., Allison, K. E., Flores, P., Gordon, M., Hess, L., \& Rogers, W. (July, 2008). Maintaining healthy behavior: How to stick with healthy eating. Presentation at Morgan \& Pottinger Company. Louisville, KY. 
Zimmerman, J. A., Mast, B. T., Miles, T., \& Markides, K. S. (November, 2007). Vascular Risk and Depression in the Hispanic Established Population for the Epidemiologic Study of the Elderly (EPESE). Poster presented at the $60^{\text {th }}$ Annual meeting of the Gerontological Society of America. San Francisco, CA.

Celebration of Undergraduate Research, Scholarship, and Creative Activity, Marist College, April 2004. "Influence of endurance performance on salivary serotonin concentration in female athletes."

Eastern Colleges Science Conference, Manhattan College, April 2004.

"Endurance exercise induced changes in salivary serotonin concentrations."

Annual Conference/American Association for the Advancement of Science, Seattle, Washington, February 2004. "Influence of endurance performance on salivary serotonin concentration in female athletes."

American Chemical Society, Mid-Hudson Chapter, Marist College, April, 2003. Third Place Award Received

"Pathological effects of platinum, palladium, and rhodium in Rattus Norvegicus tissues."

Eastern Colleges Science Conference, Ithaca College, April 2003.

First Place Award Received

"Histopathological study of the effects of platinum group metals on rats."

Psychological Undergraduate Research Conference, Marist College, December 2002.

"Gender differences in cradling due to brain hemisphere functions."

Annual Conference/American Association for the Advancement of Science, Boston, MA, March 2002.

Annals of Improbable Research ("Spoof” Journal). "The effects of television on sexual behavior."

\section{TEACHING EXPERIENCE}

Guest Lecturer - Class: Abnormal Psychology, Psychological and Brain Sciences Department: Cognitive Disorders and Life Span Issue: November, 2009

Guest Lecturer - Class: Black Psychology, Pan African Studies Department:

Psychotherapy in Black Older Adults: December, 2009

Graduate Teaching Assistant, University of Louisville, Louisville, KY

Abnormal Psychology, August, 2009 - December, 2009

Personality, January, 2010 - May, 2010

Peer Tutor, General Biology I \& General Biology II, Marist College, (Lecture and Lab), Fall 2002-2004 


\section{PROFESSIONAL SERVICES}

Graduate Student Representative, American Psychological Association, 12-II Mentoring Committee, August 2008 - Present

- Organized conference symposium on "Mentoring Across the Geropsychology Career: Perspectives from the Experts. Presented at the $62^{\text {nd }}$ Annual Scientific meeting of the Gerontological Society of America.

- Currently organizing a special section on mentoring in clinical geropsychology, Mentoring Across the Stages of Professional Development, for the Journal of Educational Gerontology. Primary author for Introduction article.

- Participate in monthly conference calls to discuss committee responsibilities and current projects

Graduate Student Peer Mentor to incoming $1^{\text {st }}$ year clinical student, Louisville, KY, July 2008 - May 2010

Senator Proxy (Executive Position), Graduate Student Council, Louisville, KY, April 2008 - August 2009

Graduate Student Council Representative, Graduate Student Council, Louisville, KY, August 2007 - August 2008

Graduate student Mentor, for DuPont Manual High School student science fair project, Louisville, KY

- Provide tutoring and supervision on statistical analyses.

\section{VOLUNTEER ACTIVITIES}

Volunteer - Team Co-Captain, 2009 Alzheimer's Association Memory Walk Raised donations for the Alzheimer's Association and participated in the walk

Volunteer - Team Co-Captain, 2008 Alzheimer's Association Memory Walk Raised donations for the Alzheimer's Association and participated in the walk

Volunteer, 2007 National Senior Games, Louisville KY

- Provided assistance in the organization of the Track and Field Senior Olympic Games.

- Signed in athletes, posted results, monitored track, passed out food and drinks, etc.

Volunteer - Team Co-Captain, 2007 Alzheimer's Association Memory Walk

- Raised donations for the Alzheimer's Association and participated in the walk

Volunteer, Highlands Adult Day Center, Louisville, KY, February 2007 - August 2008

- Spend one-on-one time with clients Friday afternoons, participate in the recreational activity with the clients 
Volunteer, Nathaniel Witherall Nursing Home, Greenwich, CT, April 2005 - September 2005

- Spent one-on-one time with the residents every Wednesday evening, participated in the recreational activity with the residents

\section{PROFESSIONAL MEMBERSHIPS}

American Psychological Association, student member, 2003-present Div. 12-2: Clinical Geropsychology

Gerontological Society of America, Student Member, 2006-present 